\title{
Standards of Medical Care in Diabetes-2010
}

American Diabetes Association

D iabetes is a chronic illness that requires continuing medical care and ongoing patient self-management education and support to prevent acute complications and to reduce the risk of long-term complications. Diabetes care is complex and requires that many issues, beyond glycemic control, be addressed. A large body of evidence exists that supports a range of interventions to improve diabetes outcomes.

These standards of care are intended to provide clinicians, patients, researchers, payors, and other interested individuals with the components of diabetes care, general treatment goals, and tools to evaluate the quality of care. While individual preferences, comorbidities, and other patient factors may require modification of goals, targets that are desirable for most patients with diabetes are provided. These standards are not intended to preclude clinical judgment or more extensive evaluation and management of the patient by other specialists as needed. For more detailed information about management of diabetes, refer to references 1-3.

The recommendations included are screening, diagnostic, and therapeutic actions that are known or believed to favorably affect health outcomes of patients with diabetes. A grading system (Table 1), developed by the American Diabetes Association (ADA) and modeled after existing methods, was used to clarify and codify the evidence that forms the basis for the recommendations. The level of evidence that supports each recommendation is listed after each recommendation using the letters A, B, C, or E.

These standards of care are revised annually by the ADA multidisciplinary Professional Practice Committee, and new evidence is incorporated. Members of the Professional Practice Committee and their disclosed conflicts of interest are listed in the Introduction. Subsequently, as with all position statements, the standards of care are reviewed and approved

\section{Originally approved 1988. Most recent review/revision October 2009}

DOI: $10.2337 / \mathrm{dc} 10-$ S011

Abbreviations: ABI, ankle-brachial index; ACCORD, Action to Control Cardiovascular Risk in Diabetes; ADAG, AlC-Derived Average Glucose Trial; ADVANCE, Action in Diabetes and Vascular Disease: Preterax and Diamicron Modified Release Controlled Evaluation; ACE, angiotensin converting enzyme; ARB, angiotensin receptor blocker; ACT-NOW, ACTos Now Study for the Prevention of Diabetes; BMI, body mass index; CBG, capillary blood glucose; CFRD, cystic fibrosis-related diabetes; CGM, continuous glucose monitoring; $\mathrm{CHD}$, coronary heart disease; $\mathrm{CHF}$, congestive heart failure; CCM, chronic care model; CKD, chronic kidney disease; CMS, Centers for Medicare and Medicaid Services; CSII, continuous subcutaneous insulin infusion; CVD, cardiovascular disease; DASH, Dietary Approaches to Stop Hypertension; DCCT, Diabetes Control and Complications Trial; DKA, diabetic ketoacidosis; DMMP, diabetes medical management plan; DPN, distal symmetric polyneuropathy; DPP, Diabetes Prevention Program; DPS, Diabetes Prevention Study; DREAM, Diabetes Reduction Assessment with Ramipril and Rosiglitazone Medication; DRS, Diabetic Retinopathy Study; DSME, diabetes self-management education; DSMT, diabetes self-management training; eAG, estimated average glucose; eGFR, estimated glomerular filtration rate; ECG, electrocardiogram; EDIC, Epidemiology of Diabetes Interventions and Complications; ERP, education recognition program; ESRD, end-stage renal disease; ETDRS, Early Treatment Diabetic Retinopathy Study; FDA, Food and Drug Administration; FPG, fasting plasma glucose; GDM, gestational diabetes mellitus; GFR, glomerular filtration rate; HAPO, Hyperglycemia and Adverse Pregnancy Outcomes; ICU, intensive care unit; IFG, impaired fasting glucose; IGT, impaired glucose tolerance; Look AHEAD, Action for Health in Diabetes; MDRD, Modification of Diet in Renal Disease; MNT, medical nutrition therapy; NDEP, National Diabetes Education Program; NGSP, National Glycohemoglobin Standardization Program; NPDR, nonproliferative diabetic retinopathy; OGTT, oral glucose tolerance test; PAD, peripheral arterial disease; PCOS, polycystic ovarian syndrome; PDR, proliferative diabetic retinopathy; PPG, postprandial plasma glucose; RAS, renin-angiotensin system; SMBG, self-monitoring of blood glucose; STOP-NIDDM, Study to Prevent Non-Insulin Dependent Diabetes; SSI, sliding scale insulin; TZD, thiazolidinedione; UKPDS, U.K. Prospective Diabetes Study; VADT, Veterans Affairs Diabetes Trial; XENDOS, XENical in the prevention of Diabetes in Obese Subjects.

(C) 2010 by the American Diabetes Association. Readers may use this article as long as the work is properly cited, the use is educational and not for profit, and the work is not altered. See http://creativecommons. org/licenses/by-nc-nd/3.0/ for details.

by the Executive Committee of ADA's Board of Directors.

\section{CLASSIFICATION AND DIAGNOSIS}

\section{A. Classification}

The classification of diabetes includes four clinical classes:

- type 1 diabetes (results from $\beta$-cell destruction, usually leading to absolute insulin deficiency)

- type 2 diabetes (results from a progressive insulin secretory defect on the background of insulin resistance)

- other specific types of diabetes due to other causes, e.g., genetic defects in $\beta$-cell function, genetic defects in insulin action, diseases of the exocrine pancreas (such as cystic fibrosis), and drugor chemical-induced diabetes (such as in the treatment of AIDS or after organ transplantation)

- gestational diabetes mellitus (GDM) (diabetes diagnosed during pregnancy)

Some patients cannot be clearly classified as having type 1 or type 2 diabetes. Clinical presentation and disease progression vary considerably in both types of diabetes. Occasionally, patients who otherwise have type 2 diabetes may present with ketoacidosis. Similarly, patients with type 1 diabetes may have a late onset and slow (but relentless) progression despite having features of autoimmune disease. Such difficulties in diagnosis may occur in children, adolescents, and adults. The true diagnosis may become more obvious over time.

\section{B. Diagnosis of diabetes}

\section{Recommendations}

For decades, the diagnosis of diabetes has been based on plasma glucose (PG) criteria, either fasting PG (FPG) or 2-h 75-g oral glucose tolerance test (OGTT) values. In 1997, the first Expert Committee on the Diagnosis and Classification of Diabetes Mellitus revised the diagnostic criteria using the observed association between 
Table $1-A D A$ evidence grading system for clinical practice recommendations

Level of

evidence

Description

A Clear evidence from well-conducted, generalizable, randomized controlled trials that are adequately powered, including

- Evidence from a well-conducted multicenter trial

- Evidence from a meta-analysis that incorporated quality ratings in the analysis

Compelling nonexperimental evidence, i.e., "all or none" rule developed by Center

for Evidence Based Medicine at Oxford

Supportive evidence from well-conducted randomized controlled trials that are adequately powered, including:

- Evidence from a well-conducted trial at one or more institutions

- Evidence from a meta-analysis that incorporated quality ratings in the analysis

B Supportive evidence from well-conducted cohort studies:

- Evidence from a well-conducted prospective cohort study or registry

- Evidence from a well-conducted meta-analysis of cohort studies

Supportive evidence from a well-conducted case-control study

C

Supportive evidence from poorly controlled or uncontrolled studies

- Evidence from randomized clinical trials with one or more major or three or more minor methodological flaws that could invalidate the results

- Evidence from observational studies with high potential for bias (such as case series with comparison to historical controls)

- Evidence from case series or case reports

Conflicting evidence with the weight of evidence supporting the recommendation

E Expert consensus or clinical experience

glucose levels and presence of retinopathy as the key factor with which to identify threshold FPG and 2-h PG levels. The committee examined data from three cross-sectional epidemiologic studies that assessed retinopathy with fundus photography or direct ophthalmoscopy and measured glycemia as FPG, 2-h PG, and $\mathrm{HbA}_{1 \mathrm{c}}$ (AlC). The studies demonstrated glycemic levels below which there was little prevalent retinopathy and above which the prevalence of retinopathy increased in an apparently linear fashion. The deciles of FPG, 2-h PG, and AlC at which retinopathy began to increase were the same for each measure within each population. The analyses helped to inform a then-new diagnostic cut point of $\geq 126 \mathrm{mg} / \mathrm{dl}(7.0 \mathrm{mmol} / \mathrm{l})$ for FPG and confirmed the long-standing diagnostic 2-h PG value of $\geq 200 \mathrm{mg} / \mathrm{dl}$ (11.1 $\mathrm{mmol} / \mathrm{l})(4)$.

ADA has not previously recommended the use of AlC for diagnosing diabetes, in part due to lack of standardization of the assay. However, AlC assays are now highly standardized, and their results can be uniformly applied both temporally and across populations. In a recent report (5), after an extensive review of both established and emerging epidemiological evidence, an international expert committee recommended the use of the AlC test to diagnose diabetes with a threshold of $\geq 6.5 \%$, and ADA affirms this decision (6). The diagnostic test should be performed using a method certified by the National Glycohemoglobin Standardization Program (NGSP) and standardized or traceable to the Diabetes Control and Complications Trial (DCCT) reference assay. Point-of-care AlC assays are not sufficiently accurate at this time to use for diagnostic purposes.

Epidemiologic datasets show a relationship between $\mathrm{AlC}$ and the risk of retinopathy similar to that which has been shown for corresponding FPG and 2-h PG thresholds. The AlC has several advantages to the FPG, including greater convenience, since fasting is not required; evidence to suggest greater preanalytical stability; and less day-to-day perturbations during periods of stress and illness. These advantages must be balanced by greater cost, limited availability of AlC testing in certain regions of the developing world, and incomplete correlation between AlC and average glucose in certain individuals. In addition, the AlC can be misleading in patients with certain forms of anemia and hemoglobinopathies. For patients with a hemoglobinopathy but normal red cell turnover, such as sickle cell trait, an AlC assay without interference from abnormal hemoglobins should be used (an updated list of AlC assays and whether abnormal hemoglobins impact them is available at www.ngsp.org/prog/ index3.html). For conditions with abnormal red cell turnover, such as pregnancy or anemias from hemolysis and iron deficiency, the diagnosis of diabetes must use glucose criteria exclusively.

The established glucose criteria for the diagnosis of diabetes (FPG and 2-h PG) remain valid. Patients with severe hyperglycemia such as those who present with severe classic hyperglycemic symptoms or hyperglycemic crisis can continue to be diagnosed when a random (or casual) $P G$ of $\geq 200 \mathrm{mg} / \mathrm{dl}$ ( $11.1 \mathrm{mmol} / \mathrm{l})$ is found. It is likely that in such cases the health care professional would also conduct an AlC test as part of the initial assessment of the severity of the diabetes and that it would be above the diagnostic cut point. However, in rapidly evolving diabetes such as the development of type 1 in some children, the AlC may not be significantly elevated despite frank diabetes.

Just as there is $<100 \%$ concordance between the FPG and 2-h PG tests, there is not perfect concordance between AlC and either glucose-based test. Analyses of National Health and Nutrition Examination Survey (NHANES) data indicate that, assuming universal screening of the undiagnosed, the AlC cut point of $\geq 6.5 \%$ identifies one-third fewer cases of undiagnosed diabetes than a fasting glucose cut point of $\geq 126 \mathrm{mg} / \mathrm{dl}(7.0 \mathrm{mmol} / \mathrm{l})(\mathrm{E}$. Gregg, personal communication). However, in practice, a large portion of the diabetic population remains unaware of their condition. Thus, the lower sensitivity of AlC at the designated cut point may well be offset by the test's greater practicality, and wider application of a more convenient test (AlC) may actually increase the number of diagnoses made.

As with most diagnostic tests, a test result diagnostic of diabetes should be repeated to rule out laboratory error, unless the diagnosis is clear on clinical grounds, such as a patient with classic symptoms of hyperglycemia or hyperglycemic crisis. It is preferable that the same test be repeated for confirmation, since there will be a greater likelihood of concurrence in this case. For example, if the AlC is $7.0 \%$ and a repeat result is $6.8 \%$, the diagnosis of diabetes is confirmed. However, there are scenarios in which results of two different tests (e.g., FPG and AlC) are available for the same patient. In this situation, if the two different tests are both above the di- 
Table 2-Criteria for the diagnosis of diabetes

1.

2.

3.

4.
AlC $\geq 6.5 \%$. The test should be performed in a laboratory using a method that is NGSP certified and standardized to the DCCT assay.*

OR

FPG $\geq 126 \mathrm{mg} / \mathrm{dl}(7.0 \mathrm{mmol} / \mathrm{l})$. Fasting is defined as no caloric intake for at least 8 h.*

OR

Two-hour plasma glucose $\geq 200 \mathrm{mg} / \mathrm{dl}$ (11.1 mmol/l) during an OGTT.

The test should be performed as described by the World Health

Organization, using a glucose load containing the equivalent of $75 \mathrm{~g}$

anhydrous glucose dissolved in water.*

OR

In a patient with classic symptoms of hyperglycemia or hyperglycemic crisis, a random plasma glucose $\geq 200 \mathrm{mg} / \mathrm{dl}$ (11.1 mmol/l).

*In the absence of unequivocal hyperglycemia, criteria $1-3$ should be confirmed by repeat testing.

agnostic threshold, the diagnosis of diabetes is confirmed.

On the other hand, if two different tests are available in an individual and the results are discordant, the test whose result is above the diagnostic cut point should be repeated, and the diagnosis is made on the basis of the confirmed test. That is, if a patient meets the diabetes criterion of the $\mathrm{AlC}$ (two results $\geq 6.5 \%$ ) but not the FPG $(<126 \mathrm{mg} / \mathrm{dl}$ or $7.0 \mathrm{mmol} / \mathrm{l})$, or vice versa, that person should be considered to have diabetes. Admittedly, in most circumstance the "nondiabetic" test is likely to be in a range very close to the threshold that defines diabetes.

Since there is preanalytic and analytic variability of all the tests, it is also possible that when a test whose result was above the diagnostic threshold is repeated, the second value will be below the diagnostic cut point. This is least likely for AlC, somewhat more likely for FPG, and most likely for the 2-h PG. Barring a laboratory error, such patients are likely to have test results near the margins of the threshold for a diagnosis. The healthcare professional might opt to follow the patient closely and repeat the testing in 3-6 months.

The current diagnostic criteria for diabetes are summarized in Table 2.

\section{Categories of increased risk for diabetes}

In 1997 and 2003, The Expert Committee on the Diagnosis and Classification of Diabetes Mellitus $(4,7)$ recognized an intermediate group of individuals whose glucose levels, although not meeting criteria for diabetes, are nevertheless too high to be considered normal. This group was defined as having impaired fasting glucose (IFG) (FPG levels of $100 \mathrm{mg} / \mathrm{dl}$ [5.6 mmol/l] to $125 \mathrm{mg} / \mathrm{dl}$ [6.9 mmol/l]) or impaired glucose tolerance (IGT) (2-h OGTT values of $140 \mathrm{mg} / \mathrm{dl}$ [7.8 mmol/l] to $199 \mathrm{mg} / \mathrm{dl}$ [11.0 mmol/l]).

Individuals with IFG and/or IGT have been referred to as having pre-diabetes, indicating the relatively high risk for the future development of diabetes. IFG and IGT should not be viewed as clinical entities in their own right but rather risk factors for diabetes as well as cardiovascular disease (CVD). IFG and IGT are associated with obesity (especially abdominal or visceral obesity), dyslipidemia with high triglycerides and/or low HDL cholesterol, and hypertension. Structured lifestyle intervention, aimed at increasing physical activity and producing $5-10 \%$ loss of body weight, and certain pharmacological agents have been demonstrated to prevent or delay the development of diabetes in people with IGT (see Table 7). It should be noted that the 2003 ADA Expert Committee report reduced the lower FPG cut point to define IFG from $110 \mathrm{mg} / \mathrm{dl}(6.1 \mathrm{mmol} / \mathrm{l})$ to 100 $\mathrm{mg} / \mathrm{dl}(5.6 \mathrm{mmol} / \mathrm{l})$, in part to make the prevalence of IFG more similar to that of IGT. However, the World Health Organization (WHO) and many other diabetes organizations did not adopt this change.

As the AlC becomes increasingly used to diagnose diabetes in individuals with risk factors, it will also identify those at high risk for developing diabetes in the future. As was the case with the glucose measures, defining a lower limit of an intermediate category of AlC is somewhat arbitrary, since risk of diabetes with any measure or surrogate of glycemia is a continuum extending well into the normal ranges. To maximize equity and efficiency of preventive interventions, such an AlC cut point, should balance the costs of false negatives (failing to identify those who are going to develop diabetes) against the costs of false positives (falsely identifying and then spending intervention resources on those who were not going to develop diabetes anyway).

Linear regression analyses of nationally representative U.S. data (NHANES 2005-2006) indicate that among the nondiabetic adult population, an FPG of $110 \mathrm{mg} / \mathrm{dl}$ corresponds to an AlC of $5.6 \%$, while an FPG of $100 \mathrm{mg} / \mathrm{dl}$ corresponds to an AlC of $5.4 \%$. Receiver operating curve analyses of these data indicate that an $\mathrm{AlC}$ value of $5.7 \%$, compared with other cut points, has the best combination of sensitivity (39\%) and specificity (91\%) to identify cases of IFG (FPG $\geq 100 \mathrm{mg} / \mathrm{dl}[5.6 \mathrm{mmol} / \mathrm{l}]$ ) (R.T. Ackerman, Personal Communication). Other analyses suggest that an AlC of $5.7 \%$ is associated with diabetes risk similar to that of the high-risk participants in the Diabetes Prevention Program (DPP) (R.T. Ackerman, personal communication). Hence, it is reasonable to consider an AlC range of 5.7-6.4\% as identifying individuals with high risk for future diabetes and to whom the term pre-diabetes may be applied (6).

As is the case for individuals found to have IFG and IGT, individuals with an AlC of 5.7-6.4\% should be informed of their increased risk for diabetes as well as CVD and counseled about effective strategies to lower their risks (see IV. PREVENTION/DELAY OF TYPE 2 DIABETES). As with glucose measurements, the continuum of risk is curvilinear, so that as AlC rises, the risk of diabetes rises disproportionately. Accordingly, interventions should be most intensive and follow-up should be particularly vigilant for those with an $\mathrm{AlC}>6.0 \%$, who should be considered to be at very high risk. However, just as an individual with a fasting glucose of $98 \mathrm{mg} / \mathrm{dl}$ (5.4 mmol/l) may not be at negligible risk for diabetes, individuals with an AlC $<5.7 \%$ may still be at risk, depending on the level of AlC and presence of other risk factors, such as obesity and family history.

Table 3-Categories of increased risk for diabetes*

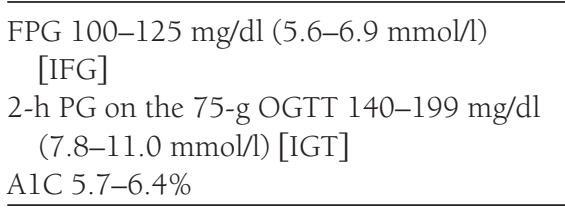

*For all three tests, risk is continuous, extending below the lower limit of the range and becoming disproportionately greater at higher ends of the range. 
Table 4-Criteria for testing for diabetes in asymptomatic adult individuals

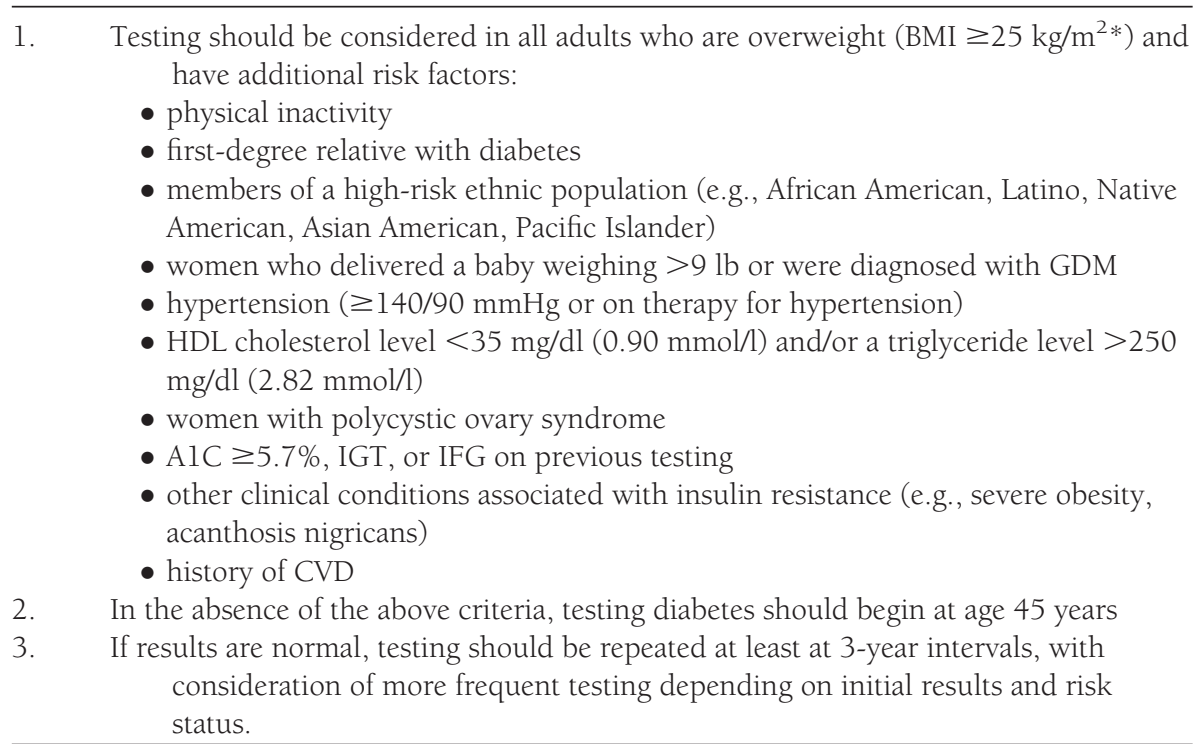

*At-risk BMI may be lower in some ethnic groups.

Table 3 summarizes the categories of increased risk for diabetes.

\section{TESTING FOR DIABETES IN ASYMPTOMATIC PATIENTS}

\section{Recommendations}

- Testing to detect type 2 diabetes and assess risk for future diabetes in asymptomatic people should be considered in adults of any age who are overweight or obese (BMI $\geq 25 \mathrm{~kg} / \mathrm{m}^{2}$ ) and who have one or more additional risk factors for diabetes (Table 4). In those without these risk factors, testing should begin at age 45 years. (B)

- If tests are normal, repeat testing should be carried out at least at 3-year intervals. (E)

- To test for diabetes or to assess risk of future diabetes, either AlC, FPG, or 2-h 75-g OGTT are appropriate. (B)

- In those identified with increased risk for future diabetes, identify and, if appropriate, treat other CVD risk factors. (B)

For many illnesses there is a major distinction between screening and diagnostic testing. However, for diabetes the same tests would be used for "screening" as for diagnosis. Type 2 diabetes has a long asymptomatic phase and significant clinical risk markers. Diabetes may be identified anywhere along a spectrum of clinical scenarios ranging from a seemingly lowrisk individual who happens to have glucose testing, to a higher-risk individual who the provider tests because of high suspicion of diabetes, to the symptomatic patient. The discussion herein is primarily framed as testing for diabetes in individuals without symptoms. Testing for diabetes will also detect individuals at increased future risk for diabetes, herein referred to as pre-diabetic.

\section{A. Testing for type 2 diabetes and risk of future diabetes in adults}

Type 2 diabetes is frequently not diagnosed until complications appear, and approximately one-fourth of all people with diabetes in the U.S. may be undiagnosed. Although the effectiveness of early identification of pre-diabetes and diabetes through mass testing of asymptomatic individuals has not been proven definitively (and rigorous trials to provide such proof are unlikely to occur), pre-diabetes and diabetes meet established criteria for conditions in which early detection is appropriate. Both conditions are common, are nificant public health burdens. There is a long presymptomatic phase before the diagnosis of type 2 diabetes is usually made. Relatively simple tests are available to detect preclinical disease (9). Additionally, the duration of glycemic burden is a strong predictor of adverse outcomes, and effective interventions exist to prevent progression of pre-diabetes to diabetes (see IV. PREVENTION/DELAY OF TYPE 2 DIABETES) and to reduce risk of complications of diabetes (see VI. PREVENTION AND MANAGEMENT OF DIABETES COMPLICATIONS). increasing in prevalence, and impose sig-
Recommendations for testing for diabetes in asymptomatic undiagnosed adults are listed in Table 4. Testing should be considered in adults of any age with $\mathrm{BMI} \geq 25 \mathrm{~kg} / \mathrm{m}^{2}$ and one or more risk factors for diabetes. Because age is a major risk factor for diabetes, testing of those without other risk factors should begin no later than at age 45 years.

Either AlC, FPG, or 2-h OGTT is appropriate for testing. The 2-h OGTT identifies people with either IFG or IGT and thus more people at increased risk for the development of diabetes and CVD. It should be noted that the two tests do not necessarily detect the same individuals (10). The efficacy of interventions for primary prevention of type 2 diabetes (11-17) has primarily been demonstrated among individuals with IGT, but not for individuals with IFG (who do not also have IGT) or those with specific AlC levels.

The appropriate interval between tests is not known (18). The rationale for the 3-year interval is that false negatives will be repeated before substantial time elapses, and there is little likelihood that an individual will develop significant complications of diabetes within 3 years of a negative test result.

Because of the need for follow-up and discussion of abnormal results, testing should be carried out within the health care setting. Community screening outside a health care setting is not recommended because people with positive tests may not seek, or have access to, appropriate follow-up testing and care. Conversely, there may be failure to ensure appropriate repeat testing for individuals who test negative. Community screening may also be poorly targeted, i.e., it may fail to reach the groups most at risk and inappropriately test those at low risk (the worried well) or even those already diagnosed $(19,20)$.

\section{B. Testing for type 2 diabetes in children}

The incidence of type 2 diabetes in adolescents has increased dramatically in the last decade, especially in minority populations (21), although the disease remains rare in the general pediatric population (22). Consistent with recommendations for adults, children and youth at increased risk for the presence or the development of type 2 diabetes should be tested within the health care setting (23). The recommendations of the ADA consensus statement on type 2 diabetes in 
Table 5-Testing for type 2 diabetes in asymptomatic children

\begin{tabular}{ll}
\hline Criteria: & Overweight (BMI $>85$ th percentile for age and sex, weight for height \\
& $>85$ th percentile, or weight $>120 \%$ of ideal for height) \\
$\begin{array}{c}\text { Plus any two of } \\
\text { the following } \\
\text { risk factors: }\end{array}$ & - Family history of type 2 diabetes in first- or second-degree relative \\
& - Race/ethnicity (Native American, African American, Latino, Asian \\
& - Signs of insulin resistance or conditions associated with insulin \\
& resistance (acanthosis nigricans, hypertension, dyslipidemia, \\
& polycystic ovary syndrome, or small for gestational age \\
& birthweight) \\
Age of & Maternal history of diabetes or GDM during the child's gestation \\
initiation: & Age 10 years or at onset of puberty, if puberty occurs at a younger \\
Frequency: & age
\end{tabular}

children and youth, with some modifications, are summarized in Table 5.

\section{Screening for type 1 diabetes}

Generally, people with type 1 diabetes present with acute symptoms of diabetes and markedly elevated blood glucose levels, and most cases are diagnosed soon after the onset of hyperglycemia. However, evidence from type 1 diabetes prevention studies suggests that measurement of islet autoantibodies identifies individuals who are at risk for developing type 1 diabetes. Such testing may be appropriate in high-risk individuals, such as those with prior transient hyperglycemia or those who have relatives with type 1 diabetes, in the context of clinical research studies (see, for example, http://www2. diabetestrialnet.org). Widespread clinical testing of asymptomatic low-risk individuals cannot currently be recommended, as it would identify very few individuals in the general population who are at risk. Individuals who screen positive should be counseled about their risk of developing diabetes. Clinical studies are being conducted to test various methods of preventing type 1 diabetes or reversing early type 1 diabetes in those with evidence of autoimmunity.

\section{DETECTION AND DIAGNOSIS OF GDM}

\section{Recommendations}

- Screen for GDM using risk factor analysis and, if appropriate, an OGTT. (C)

- Women with GDM should be screened for diabetes 6-12 weeks postpartum and should be followed up with subsequent screening for the development of diabetes or pre-diabetes. (E)

For many years, GDM has been defined as any degree of glucose intolerance with on- set or first recognition during pregnancy (4). Although most cases resolve with delivery, the definition applied whether the condition persisted after pregnancy and did not exclude the possibility that unrecognized glucose intolerance may have antedated or begun concomitantly with the pregnancy. This definition facilitated a uniform strategy for detection and classification of GDM, but its limitations were recognized for many years. As the ongoing epidemic of obesity and diabetes has led to more type 2 diabetes in women of childbearing age, the number of pregnant women with undiagnosed type 2 diabetes has increased (24). After deliberations in 2008-2009, the International Association of Diabetes and Pregnancy Study Groups (IADPSG), an international consensus group with representatives from multiple obstetrical and diabetes organizations, including ADA, recommended that high-risk women found to have diabetes at their initial prenatal visit using standard criteria (Table 2 ) receive a diagnosis of overt, not gestational, diabetes.

Approximately $7 \%$ of all pregnancies (ranging from 1 to $14 \%$ depending on the population studied and the diagnostic tests used) are complicated by GDM, resulting in more than 200,000 cases annually.

Because of the risks of GDM to the mother and neonate, screening and diagnosis are warranted. Current screening and diagnostic strategies, based on the 2004 ADA position statement on GDM (25), are outlined in Table 6.

Results of the Hyperglycemia and Adverse Pregnancy Outcomes (HAPO) study (26), a large-scale ( $\sim 25,000$ pregnant women) multinational epidemiologic study, demonstrated that risk of adverse maternal, fetal, and neonatal outcomes continuously increased as a function of maternal glycemia at 24-28 weeks, even within ranges previously considered normal for pregnancy. For most complications there was no threshold for risk. These results have led to careful reconsideration of the diagnostic criteria for GDM. The IADPSG recommended that all women not known to have prior diabetes undergo a 75-g OGTT at 24-28 weeks of gestation. The group developed diagnostic cut points for the fasting, $1-\mathrm{h}$, and 2-h PG measurements that conveyed an odds ratio for adverse outcomes of at least 1.75 compared with women with the mean glucose levels in the HAPO study.

At the time of this update to the Standards of Medical Care in Diabetes, ADA is planning to work with U.S. obstetrical organizations to consider adoption of the IADPSG diagnostic criteria and to discuss the implications of this change. While this change will significantly increase the prevalence of GDM, there is mounting evidence that treating even mild GDM reduces morbidity for both mother and baby (27).

Because women with a history of GDM have a greatly increased subsequent risk for diabetes (28), they should be screened for diabetes 6-12 weeks postpartum, using nonpregnant OGTT criteria, and should be followed up with subsequent screening for the development of diabetes or pre-diabetes, as outlined in II. TESTING FOR DIABETES IN ASYMPTOMATIC PATIENTS. Information on the National Diabetes Education Program (NDEP) campaign to prevent type 2 diabetes in women with GDM can be found at http://ndep.nih.gov/media/ NeverTooEarly_Tipsheet.pdf.

\section{PREVENTION/DELAY OF TYPE 2 DIABETES}

\section{Recommendations}

- Patients with IGT (A), IFG (E), or an AlC of 5.7-6.4\% (E) should be referred to an effective ongoing support program for weight loss of 5-10\% of body weight and an increase in physical activity of at least $150 \mathrm{~min} /$ week of moderate activity such as walking.

- Follow-up counseling appears to be important for success. (B)

- Based on potential cost savings of diabetes prevention, such counseling should be covered by third-party payors. (E)

- In addition to lifestyle counseling, metformin may be considered in those who are at very high risk for developing diabetes (combined IFG and IGT plus 
Table 6-Screening for and diagnosis of GDM

Carry out diabetes risk assessment at the first prenatal visit.

Women at very high risk should be screened for diabetes as soon as possible after the confirmation of pregnancy. Criteria for very high risk are:

- Severe obesity

- Prior history of GDM or delivery of large-for-gestational-age infant

- Presence of glycosuria

- Diagnosis of PCOS

- Strong family history of type 2 diabetes

Screening/diagnosis at this stage of pregnancy should use standard diagnostic testing (Table 2).

All women of greater than low risk of GDM, including those above not found to have diabetes early in pregnancy, should undergo GDM testing at 24-28 weeks of gestation. Lowrisk status, which does not require GDM screening, is defined as women with ALL of the following characteristics:

- Age $<25$ years

- Weight normal before pregnancy

- Member of an ethnic group with a low prevalence of diabetes

- No known diabetes in first-degree relatives

- No history of abnormal glucose tolerance

- No history of poor obstetrical outcome

Two approaches may be followed for GDM screening at 24-28 weeks:

1. Two-step approach:

A. Perform initial screening by measuring plasma or serum glucose $1 \mathrm{~h}$ after a $50-\mathrm{g}$ load of $\geq 140 \mathrm{mg} / \mathrm{dl}$ identifies $\sim 80 \%$ of women with GDM, while the sensitivity is further increased to $\sim 90 \%$ by a threshold of $\geq 130 \mathrm{mg} / \mathrm{dl}$.

B. Perform a diagnostic 100-g OGTT on a separate day in women who exceed the chosen threshold on 50 -g screening.

2. One-step approach (may be preferred in clinics with high prevalence of GDM): Perform a diagnostic 100-g OGTT in all women to be tested at 24-28 weeks.

The 100-g OGTT should be performed in the morning after an overnight fast of at least 8 h.

To make a diagnosis of GDM, at least two of the following plasma glucose values must be found:

- Fasting $\geq 95 \mathrm{mg} / \mathrm{dl}$

- $1-\mathrm{h} \geq 180 \mathrm{mg} / \mathrm{dl}$

- $2-\mathrm{h} \geq 155 \mathrm{mg} / \mathrm{dl}$

- $3-\mathrm{h} \geq 140 \mathrm{mg} / \mathrm{dl}$

other risk factors such as AlC $>6 \%$, hypertension, low HDL cholesterol, elevated triglycerides, or family history of diabetes in a first-degree relative) and who are obese and under 60 years of age. (E)

- Monitoring for the development of diabetes in those with pre-diabetes should be performed every year. (E)

Randomized controlled trials have shown that individuals at high risk for developing diabetes (those with IFG, IGT, or both) can be given interventions that significantly decrease the rate of onset of diabetes (11-17). These interventions include intensive lifestyle modification programs that have been shown to be very effective (58\% reduction after 3 years) and use of the pharmacologic agents metformin, $\alpha$-glucosidase inhibitors, orlistat, and thiazolidinediones, each of which has been shown to decrease incident diabetes to various degrees. A summary of major diabetes prevention trials is shown in Table 7 .

Two studies of lifestyle intervention have shown persistent reduction in the role of conversion to type 2 diabetes with 3 years (29) to 14 years (30) of postintervention follow-up.

Based on the results of clinical trials and the known risks of progression of pre-diabetes to diabetes, an ADA Consensus Development Panel (36) concluded that people with IGT and/or IFG should be counseled on lifestyle changes with goals similar to those of the DPP (5-10\% weight loss and moderate physical activity of $\sim 30 \mathrm{~min} /$ day). Regarding the more difficult issue of drug therapy for diabetes prevention, the consensus panel felt that metformin should be the only drug considered for use in diabetes prevention. For other drugs, the issues of cost, side effects, and lack of persistence of effect in some studies led the panel to not recommend use for diabetes prevention. Metformin use was recommended only for veryhigh-risk individuals (those with combined IGT and IFG who are obese and have at least one other risk factor for diabetes) who are under 60 years of age. In addition, the panel highlighted the evidence that in the DPP, metformin was most effective compared with lifestyle in individuals with $\mathrm{BMI} \geq 35 \mathrm{~kg} / \mathrm{m}^{2}$ and those under age 60 years.

\section{DIABETES CARE}

\section{A. Initial evaluation}

A complete medical evaluation should be performed to classify the diabetes, detect the presence of diabetes complications, review previous treatment and glycemic control in patients with established diabetes, assist in formulating a management plan, and provide a basis for continuing care. Laboratory tests appropriate to the evaluation of each patient's medical condition should be performed. A focus on the components of comprehensive care (Table 8) will assist the health care team to ensure optimal management of the patient with diabetes.

\section{B. Management}

People with diabetes should receive medical care from a physician-coordinated team. Such teams may include, but are not limited to, physicians, nurse practitioners, physician's assistants, nurses, dietitians, pharmacists, and mental health professionals with expertise and a special interest in diabetes. It is essential in this collaborative and integrated team approach that individuals with diabetes assume an active role in their care.

The management plan should be formulated as a collaborative therapeutic alliance among the patient and family, the physician, and other members of the health care team. A variety of strategies and techniques should be used to provide adequate education and development of problem-solving skills in the various aspects of diabetes management. Implementation of the management plan requires that each aspect is understood and agreed to by the patient and the care providers and that the goals and treatment plan are reasonable. Any plan should recognize diabetes self-management education (DSME) and on-going diabetes support as an integral component 


\begin{tabular}{|c|c|c|c|c|c|c|c|c|}
\hline$\underline{\text { Study (ref.) }}$ & $n$ & Population & $\begin{array}{c}\text { Mean } \\
\text { age } \\
\text { (years) } \\
\end{array}$ & $\begin{array}{c}\text { Duration } \\
\text { (years) }\end{array}$ & $\begin{array}{c}\text { Intervention } \\
\text { (daily dose) }\end{array}$ & $\begin{array}{c}\text { Incidence in } \\
\text { control } \\
\text { subjects } \\
\text { (\%/year) }\end{array}$ & $\begin{array}{c}\text { Relative risk } \\
\text { reduction (\%) } \\
(95 \% \mathrm{CI})\end{array}$ & $\begin{array}{c}\text { 3-Year } \\
\text { number } \\
\text { needed to } \\
\text { treat* }^{*}\end{array}$ \\
\hline \multicolumn{9}{|l|}{ Lifestyle } \\
\hline Finnish DPS (12) & 522 & $\mathrm{IGT}, \mathrm{BMI} \geq 25 \mathrm{~kg} / \mathrm{m}^{2}$ & 55 & 3.2 & I-D\&E & 6 & $58(30-70)$ & 8.5 \\
\hline $\operatorname{DPP}(11)$ & $2,161 \dagger$ & $\begin{array}{l}\mathrm{IGT}, \mathrm{BMI} \geq 24 \mathrm{~kg} / \mathrm{m}^{2} \\
\quad \mathrm{FPG}>5.3 \mathrm{mmol} / \mathrm{l}\end{array}$ & 51 & 3 & I-D\&E & 10.4 & $58(48-66)$ & 6.9 \\
\hline Da Qing (13) & $259 \dagger$ & $\begin{array}{l}\text { IGT (randomized } \\
\text { groups) }\end{array}$ & 45 & 6 & G-D\&E & 14.5 & $38(14-56)$ & 7.9 \\
\hline Toranomon Study (31) & 458 & $\begin{array}{l}\text { IGT (men), BMI }=24 \\
\text { kg/m } \mathrm{m}^{2}\end{array}$ & $\sim 55$ & 4 & I-D\&E & 2.4 & $67(P<0.043) \ddagger$ & 20.6 \\
\hline Indian DPP (17) & $269 \dagger$ & IGT & 46 & 2.5 & I-D\&E & 23 & $29(21-37)$ & 6.4 \\
\hline \multicolumn{9}{|l|}{ Medications } \\
\hline $\mathrm{DPP}(11)$ & $2,155 \dagger$ & $\begin{array}{c}\mathrm{IGT}, \mathrm{BMI}>24 \mathrm{~kg} / \mathrm{m}^{2} \\
\quad \mathrm{FPG}>5.3 \mathrm{mmol} / \mathrm{l}\end{array}$ & 51 & 2.8 & $\begin{array}{l}\text { Metformin } \\
\quad(1,700 \\
\mathrm{mg})\end{array}$ & 10.4 & $31(17-43)$ & 13.9 \\
\hline Indian DPP (17) & $269 \dagger$ & IGT & 46 & 2.5 & $\begin{array}{l}\text { Metformin } \\
\quad(500 \mathrm{mg})\end{array}$ & 23 & $26(19-35)$ & 6.9 \\
\hline STOP NIDDM (15) & 1,419 & $\begin{array}{l}\mathrm{IGT}, \mathrm{FPG}>5.6 \\
\mathrm{mmol} / \mathrm{l}\end{array}$ & 54 & 3.2 & $\begin{array}{l}\text { Acarbose } \\
\quad(300 \mathrm{mg})\end{array}$ & 12.4 & $25(10-37)$ & 9.6 \\
\hline XENDOS (32) & 3,277 & $\mathrm{BMI}>30 \mathrm{~kg} / \mathrm{m}^{2}$ & 43 & 4 & $\begin{array}{l}\text { Orlistat (360 } \\
\text { mg) }\end{array}$ & 2.4 & $37(14-54)$ & 45.5 \\
\hline DREAM (16) & 5,269 & IGT or IFG & 55 & 3.0 & $\begin{array}{l}\text { Rosiglitazone } \\
\quad(8 \mathrm{mg})\end{array}$ & 9.1 & $60(54-65)$ & 6.9 \\
\hline Voglibose Ph-3 (33) & 1,780 & IGT & 56 & 3.0 (1-year Rx) & $\begin{array}{r}\text { Vogliobose } \\
(0.2 \mathrm{mg})\end{array}$ & 12.0 & $40(18-57)$ & $\begin{array}{l}21 \text { (1-year } \\
\mathrm{Rx} \text { ) }\end{array}$ \\
\hline ACT-NOW (34) & 602 & IGT or IFG & 52 & 2.6 & $\begin{array}{c}\text { Pioglitizone } \\
\text { (45 mg) }\end{array}$ & 6.8 & $81(61-91)$ & 6.3 \\
\hline
\end{tabular}

Modified and reprinted with permission (35). Percentage points: *Number needed to treat to prevent 1 case of diabetes, standardized for a 3-year period to improve comparisons across studies. $\uparrow$ Number of participants in the indicated comparisons, not necessarily in entire study. $¥$ Calculated from information in the article. ACT-NOW, ACTos Now Study for the Prevention of Diabetes; DPP, Diabetes Prevention Program; DPS, Diabetes Prevention Study; DREAM, Diabetes Reduction Assessment with Ramipril and Rosiglitazone Medication; STOP NIDDM, Study to Prevent Non-Insulin Dependent Diabetes; XENDOS, Xenical in the prevention of Diabetes in Obese Subjects. I, individual; G, group; D\&E, diet and exercise.

of care. In developing the plan, consideration should be given to the patient's age, school or work schedule and conditions, physical activity, eating patterns, social situation and cultural factors, and presence of complications of diabetes or other medical conditions.

\section{Glycemic control}

\section{Assessment of glycemic control}

Two primary techniques are available for health providers and patients to assess the effectiveness of the management plan on glycemic control: patient self-monitoring of blood glucose (SMBG) or interstitial glucose and AlC.

\section{a. Glucose monitoring}

\section{Recommendations}

- SMBG should be carried out three or more times daily for patients using mul- tiple insulin injections or insulin pump therapy. (A)

- For patients using less frequent insulin injections, noninsulin therapies, or medical nutrition therapy (MNT) alone, SMBG may be useful as a guide to the success of therapy. (E)

- To achieve postprandial glucose targets, postprandial SMBG may be appropriate. (E)

- When prescribing SMBG, ensure that patients receive initial instruction in, and routine follow-up evaluation of, SMBG technique and using data to adjust therapy. (E)

- Continuous glucose monitoring (CGM) in conjunction with intensive insulin regimens can be a useful tool to lower AlC in selected adults (age $\geq 25$ years) with type 1 diabetes (A).

- Although the evidence for A1C lowering is less strong in children, teens, and younger adults, CGM may be helpful in these groups. Success correlates with adherence to ongoing use of the device. (C)

- CGM may be a supplemental tool to SMBG in those with hypoglycemia unawareness and/or frequent hypoglycemic episodes. (E)

The ADA consensus and position statements on SMBG provide a comprehensive review of the subject $(37,38)$. Major clinical trials of insulin-treated patients that demonstrated the benefits of intensive glycemic control on diabetes complications have included SMBG as part of multifactorial interventions, suggesting that SMBG is a component of effective therapy. SMBG allows patients to evaluate their individual response to therapy and assess whether glycemic targets are being achieved. Results of SMBG can be useful in preventing hypoglycemia and adjusting medications (particularly prandial insulin doses), MNT, and physical activity. 
Table 8-Components of the comprehensive diabetes evaluation

Medical history

- Age and characteristics of onset of diabetes (e.g., DKA, asymptomatic laboratory finding)

- Eating patterns, physical activity habits, nutritional status, and weight history; growth and development in children and adolescents

- Diabetes education history

- Review of previous treatment regimens and response to therapy (AlC records)

Current treatment of diabetes, including medications, meal plan, physical activity patterns, and results of glucose monitoring and patient's use of data

- DKA frequency, severity, and cause

- Hypoglycemic episodes

- Hypoglycemia awareness

- Any severe hypoglycemia: frequency and cause

- History of diabetes-related complications

- Microvascular: retinopathy, nephropathy, neuropathy (sensory, including history of foot lesions; autonomic, including sexual dysfunction and gastroparesis)

- Macrovascular: CHD, cerebrovascular disease, PAD

- Other: psychosocial problems*, dental disease*

Physical examination

- Height, weight, BMI

- Blood pressure determination, including orthostatic measurements when indicated

- Fundoscopic examination*

- Thyroid palpation

- Skin examination (for acanthosis nigricans and insulin injection sites)

- Comprehensive foot examination:

- Inspection

- Palpation of dorsalis pedis and posterior tibial pulses

- Presence/absence of patellar and Achilles reflexes

- Determination of proprioception, vibration, and monofilament sensation

Laboratory evaluation

- AlC, if results not available within past 2-3 months

- If not performed/available within past year:

- Fasting lipid profile, including total, LDL- and HDL cholesterol and triglycerides

- Liver function tests

- Test for urine albumin excretion with spot urine albumin/creatinine ratio

- Serum creatinine and calculated GFR

- TSH in type 1 diabetes, dyslipidemia, or women over age 50 years

Referrals

- Annual dilated eye exam

- Family planning for women of reproductive age

- Registered dietitian for MNT

- DSME

- Dental examination

- Mental health professional, if needed

* See appropriate referrals for these categories.

The frequency and timing of SMBG should be dictated by the particular needs and goals of the patient. SMBG is especially important for patients treated with insulin in order to monitor for and prevent asymptomatic hypoglycemia and hyperglycemia. For most patients with type 1 diabetes and pregnant women taking insulin, SMBG is recommended three or more times daily. For these populations, significantly more frequent testing may be required to reach AlC targets safely without hypoglycemia. The optimal frequency and timing of SMBG for patients with type 2 diabetes on noninsulin therapy is un- clear. A meta-analysis of SMBG in noninsulin-treated patients with type 2 diabetes concluded that some regimen of SMBG was associated with a reduction in AlC of $0.4 \%$. However, many of the studies in this analysis also included patient education with diet and exercise counseling and, in some cases, pharmacologic intervention, making it difficult to assess the contribution of SMBG alone to improved control (39). Several recent trials have called into question the clinical utility and cost-effectiveness of routine SMBG in non-insulin-treated patients $(40-42)$.

Because the accuracy of SMBG is in- strument and user dependent (43), it is important to evaluate each patient's monitoring technique, both initially and at regular intervals thereafter. In addition, optimal use of SMBG requires proper interpretation of the data. Patients should be taught how to use the data to adjust food intake, exercise, or pharmacological therapy to achieve specific glycemic goals, and these skills should be reevaluated periodically.

CGM through the measurement of interstitial glucose (which correlates well with $P G)$ is available. These sensors require calibration with SMBG, and the latter are still recommended for making acute treatment decisions. CGM devices also have alarms for hypo- and hyperglycemic excursions. Small studies in selected patients with type 1 diabetes have suggested that CGM use reduces the time spent in hypo- and hyperglycemic ranges and may modestly improve glycemic control. A larger 26-week randomized trial of 322 type 1 diabetic patients showed that adults age 25 years and older using intensive insulin therapy and CGM experienced a $0.5 \%$ reduction in $\mathrm{AlC}$ (from $\sim 7.6$ to $7.1 \%$ ) compared with usual intensive insulin therapy with SMBG (44). Sensor use in children, teens, and adults to age 24 years did not result in significant AlC lowering, and there was no significant difference in hypoglycemia in any group. Importantly, the greatest predictor of AlC lowering in this study for all agegroups was frequency of sensor use, which was lower in younger age-groups. In a smaller randomized controlled trial of 129 adults and children with baseline AlC $<7.0 \%$, outcomes combining AlC and hypoglycemia favored the group using CGM, suggesting that CGM is also beneficial for individuals with type 1 diabetes who have already achieved excellent control with AlC $<7.0 \%$ (45). Although CGM is an evolving technology, emerging data suggest that it may offer benefit in appropriately selected patients who are motivated to wear it most of the time. CGM may be particularly useful in those with hypoglycemia unawareness and/or frequent episodes of hypoglycemia, and studies in this area are ongoing.

\section{b. A1C}

\section{Recommendations}

- Perform the AlC test at least two times a year in patients who are meeting treatment goals (and who have stable glycemic control). (E) 
- Perform the AlC test quarterly in patients whose therapy has changed or who are not meeting glycemic goals. (E)

- Use of point-of-care testing for AlC allows for timely decisions on therapy changes, when needed. (E)

Because $\mathrm{AlC}$ is thought to reflect average glycemia over several months (43) and has strong predictive value for diabetes complications $(11,46)$, AlC testing should be performed routinely in all patients with diabetes, at initial assessment and then as part of continuing care. Measurement approximately every 3 months determines whether a patient's glycemic targets have been reached and maintained. For any individual patient, the frequency of AlC testing should be dependent on the clinical situation, the treatment regimen used, and the judgment of the clinician. Some patients with stable glycemia well within target may do well with testing only twice per year, while unstable or highly intensively managed patients (e.g., pregnant type 1 diabetic women) may be tested more frequently than every 3 months. The availability of the AlC result at the time that the patient is seen (point-of-care testing) has been reported to result in increased intensification of therapy and improvement in glycemic control $(47,48)$.

The AlC test is subject to certain limitations. Conditions that affect erythrocyte turnover (hemolysis, blood loss) and hemoglobin variants must be considered, particularly when the $\mathrm{AlC}$ result does not correlate with the patient's clinical situation (43). In addition, AlC does not provide a measure of glycemic variability or hypoglycemia. For patients prone to glycemic variability (especially type 1 diabetic patients, or type 2 diabetic patients with severe insulin deficiency), glycemic control is best judged by the combination of results of SMBG testing and the AlC. The AlC may also serve as a check on the accuracy of the patient's meter (or the patient's reported SMBG results) and the adequacy of the SMBG testing schedule.

Table 9 contains the correlation between AlC levels and mean PG levels based on data from the international AlCDerived Average Glucose (ADAG) trial using frequent SMBG and CGM in 507 adults (83\% Caucasian) with type 1, type 2 , and no diabetes (49). ADA and the American Association of Clinical Chemists have determined that the correlation $(r=0.92)$ is strong enough to justify re-
Table 9-Correlation of A1C with average glucose

\begin{tabular}{lcc} 
& \multicolumn{2}{c}{ Mean plasma glucose } \\
\cline { 2 - 3 } AlC (\%) & $\mathrm{mg} / \mathrm{dl}$ & $\mathrm{mmol} / \mathrm{l}$ \\
\hline 6 & 126 & 7.0 \\
7 & 154 & 8.6 \\
8 & 183 & 10.2 \\
9 & 212 & 11.8 \\
10 & 240 & 13.4 \\
11 & 269 & 14.9 \\
12 & 298 & 16.5 \\
\hline
\end{tabular}

These estimates are based on ADAG data of $\sim 2,700$ glucose measurements over 3 months per $\mathrm{AlC}$ measurement in 507 adults with type 1, type 2, and no diabetes. The correlation between $\mathrm{AlC}$ and average glucose was 0.92 (49). A calculator for converting $\mathrm{AlC}$ results into estimated average glucose (eAG), in either $\mathrm{mg} / \mathrm{dl}$ or $\mathrm{mmol} / \mathrm{h}$, is available at http://professional.diabetes.org/eAG.

porting both an AlC result and an estimated average glucose (eAG) result when a clinician orders the AlC test. In previous versions of the Standards of Medical Care in Diabetes, the table describing the correlation between AlC and mean glucose was derived from relatively sparse data (one seven-point profile over 1 day per AlC reading) in the primarily Caucasian type 1 participants in the DCCT (50). Clinicians should note that the numbers in the table are now different, as they are based on $\sim 2,800$ readings per AlC in the ADAG trial.

In the ADAG trial, there were no significant differences among racial and ethnic groups in the regression lines between $\mathrm{AlC}$ and mean glucose, although there was a trend toward a difference between Africans/African Americans participants and Caucasians that might have been significant had more Africans/African Americans been studied. A recent study comparing AlC to CGM data in 48 type 1 diabetic children found a highly statistically significant correlation between AlC and mean blood glucose, although the correlation ( $r=0.7)$ was significantly lower than in the ADAG trial (51). Whether there are significant differences in how $\mathrm{AlC}$ relates to average glucose in children or in African American patients is an area for further study. For the time being, the question has not led to different recommendations about testing $\mathrm{AlC}$ or different interpretations of the clinical meaning of given levels of $\mathrm{AlC}$ in those populations.

For patients in whom AlC/eAG and measured blood glucose appear discrep- ant, clinicians should consider the possibilities of hemoglobinopathy or altered red cell turnover and the options of more frequent and/or different timing of SMBG or use of CGM. Other measures of chronic glycemia such as fructosamine are available, but their linkage to average glucose and their prognostic significance are not as clear as is the case for AlC.

\section{Glycemic goals in adults}

- Lowering A1C to below or around 7\% has been shown to reduce microvascular and neuropathic complications of type 1 and type 2 diabetes. Therefore, for microvascular disease prevention, the $\mathrm{AlC}$ goal for nonpregnant adults in general is $<7 \%$. (A)

- In type 1 and type 2 diabetes, randomized controlled trials of intensive versus standard glycemic control have not shown a significant reduction in CVD outcomes during the randomized portion of the trials. Long-term follow-up of the DCCT and UK Prospective Diabetes Study (UKPDS) cohorts suggests that treatment to AlC targets below or around $7 \%$ in the years soon after the diagnosis of diabetes is associated with long-term reduction in risk of macrovascular disease. Until more evidence becomes available, the general goal of $<7 \%$ appears reasonable for many adults for macrovascular risk reduction. (B)

- Subgroup analyses of clinical trials such as the DCCT and UKPDS, and evidence for reduced proteinuria in the Action in Diabetes and Vascular Disease: Preterax and Diamicron Modified Release Controlled Evaluation (ADVANCE) trial suggest a small but incremental benefit in microvascular outcomes with AlC values closer to normal. Therefore, for selected individual patients, providers might reasonably suggest even lower AlC goals than the general goal of $<7 \%$, if this can be achieved without significant hypoglycemia or other adverse effects of treatment. Such patients might include those with short duration of diabetes, long life expectancy, and no significant CVD. (B)

- Conversely, less-stringent AlC goals than the general goal of $<7 \%$ may be appropriate for patients with a history of severe hypoglycemia, limited life expectancy, advanced microvascular or macrovascular complications, and extensive comorbid conditions and those with longstanding diabetes in whom the general goal is difficult to attain de- 
spite diabetes self-management education, appropriate glucose monitoring, and effective doses of multiple glucoselowering agents including insulin. (C)

Glycemic control is fundamental to the management of diabetes. The DCCT, a prospective, randomized, controlled trial of intensive versus standard glycemic control in patients with relatively recently diagnosed type 1 diabetes, showed definitively that improved glycemic control is associated with significantly decreased rates of microvascular (retinopathy and nephropathy) as well as neuropathic complications (53). Follow-up of the DCCT cohorts in the Epidemiology of Diabetes Interventions and Complications (EDIC) study has shown persistence of this effect in previously intensively treated subjects, even though their glycemic control has been equivalent to that of previous standard arm subjects during follow-up $(54,55)$.

In type 2 diabetes, the Kumamoto study $(56)$ and the UKPDS $(57,58)$ demonstrated significant reductions in microvascular and neuropathic complications with intensive therapy. Similar to the DCCT-EDIC findings, long-term follow-up of the UKPDS cohort has recently demonstrated a "legacy effect" of early intensive glycemic control on long-term rates of microvascular complications, even with loss of glycemic separation between the intensive and standard cohorts after the end of the randomized controlled trial (59). The more recent Veterans Affairs Diabetes Trial (VADT) in type 2 diabetes also showed significant reductions in albuminuria with intensive (achieved median AlC 6.9\%) compared with standard glycemic control but no difference in retinopathy and neuropathy $(60,61)$.

In each of these large randomized prospective clinical trials, treatment regimens that reduced average AlC to $7 \%$ ( $1 \%$ above the upper limits of normal) were associated with fewer markers of long-term microvascular complications; however, intensive control was found to increase the risk of severe hypoglycemia and led to weight gain $(46,60,62)$.

Epidemiological analyses of the DCCT and UKPDS $(46,53)$ demonstrate a curvilinear relationship between $\mathrm{AlC}$ and microvascular complications. Such analyses suggest that, on a population level, the greatest number of complications will be averted by taking patients from very poor control to fair or good control. These analyses also suggest that further lowering of AlC from 7 to $6 \%$ is associated with further reduction in the risk of microvascular complications, albeit the absolute risk reductions become much smaller. The ADVANCE study of intensive versus standard glycemic control in type 2 diabetes found a statistically significant reduction in albuminuria with an AlC target of $<6.5 \%$ (achieved median AlC $6.3 \%)$ compared with standard therapy achieving a median AlC of 7.0\% (63). Given the substantially increased risk of hypoglycemia (particularly in those with type 1 diabetes, but also in the recent type 2 diabetes trials described below), the concerning mortality findings in the Action to Control Cardiovascular Risk in Diabetes (ACCORD) trial described below and the relatively much greater effort required to achieve near-normoglycemia, the risks of lower targets may outweigh the potential benefits on microvascular complications on a population level. However, selected individual patients, especially those with little comorbidity and long life expectancy (who may reap the benefits of further lowering glycemia below 7\%) may, at patient and provider judgment, adopt glycemic targets as close to normal as possible as long as significant hypoglycemia does not become a barrier.

Whereas many epidemiologic studies and meta-analyses $(64,65)$ have clearly shown a direct relationship between AlC and CVD, the potential of intensive glycemic control to reduce CVD has been less clearly defined. In the DCCT, there was a trend toward lower risk of CVD events with intensive control (risk reduction $41 \%, 95 \%$ CI 10-68\%), but the number of events was small. However, 9-year post-DCCT follow-up of the cohort has shown that participants previously randomized to the intensive arm had a $42 \%$ reduction $(P=0.02)$ in CVD outcomes and a $57 \%$ reduction $(P=0.02)$ in the risk of nonfatal myocardial infarction (MI), stroke, or CVD death compared with participants previously in the standard arm (66). The benefit of intensive glycemic control in this type 1 diabetic cohort has recently been shown to persist for up to 30 years (67).

The UKPDS trial of type 2 diabetes observed a $16 \%$ reduction in cardiovascular complications (combined fatal or nonfatal MI and sudden death) in the intensive glycemic control arm, although this difference was not statistically significant $(P=0.052)$, and there was no suggestion of benefit on other CVD outcomes such as stroke. In an epidemiologic analysis of the study cohort, a continuous association was observed such that for every percentage point lower median on-study AlC (e.g., 8-7\%), there was a statistically significant $18 \%$ reduction in CVD events, again with no glycemic threshold. A recent report of 10 years of follow-up of the UKPDS cohort described, for the participants originally randomized to intensive glycemic control compared with those randomized to conventional glycemic control, long-term reductions in MI (15\% with sulfonylurea or insulin as initial pharmacotherapy, 33\% with metformin as initial pharmacotherapy, both statistically significant) and in all-cause mortality (13 and $27 \%$, respectively, both statistically significant) (59).

Because of ongoing uncertainty regarding whether intensive glycemic control can reduce the increased risk of CVD events in people with type 2 diabetes, several large long-term trials were launched in the past decade to compare the effects of intensive versus standard glycemic control on CVD outcomes in relatively high-risk participants with established type 2 diabetes. In 2008, results of three large trials (ACCORD, ADVANCE, and VADT) suggested no significant reduction in CVD outcomes with intensive glycemic control in these populations. Details of these three studies are shown in Table 10, and their results and implications are reviewed more extensively in a recent ADA position statement (52).

The ACCORD study randomized 10,251 participants with either history of a CVD event or significant CVD risk to a strategy of intensive glycemic control (target AlC $<6.0 \%$ ) or standard glycemic control (AlC target 7.0-7.9\%). Investigators used multiple glycemic medications in both arms. From a baseline median AlC of $8.1 \%$, the intensive arm reached a median AlC of $6.4 \%$ within 12 months of randomization, while the standard group reached a median AlC of $7.5 \%$. Other risk factors were treated aggressively and equally in both groups. The intensive glycemic control group had more use of insulin in combination with multiple oral agents, significantly more weight gain, and more episodes of severe hypoglycemia than the standard group.

In early 2008, the glycemic control arm of ACCORD was halted on the recommendation of the study's data safety monitoring board due to the finding of an increased rate of mortality in the intensive arm compared with the standard arm 


\begin{tabular}{|c|c|c|c|}
\hline & ACCORD & ADVANCE & VADT \\
\hline \multicolumn{4}{|l|}{ Participant characteristics } \\
\hline$n$ & 10,251 & 11,140 & 1,791 \\
\hline Mean age (years) & 62 & 66 & 60 \\
\hline Duration of diabetes (years) & 10 & 8 & 11.5 \\
\hline History of CVD (\%) & 35 & 32 & 40 \\
\hline Median baseline AlC (\%) & 8.1 & 7.2 & 9.4 \\
\hline On insulin at baseline (\%) & 35 & 1.5 & 52 \\
\hline \multicolumn{4}{|l|}{ Protocol characteristics } \\
\hline AlC goals (\%) (I vs. S)* & $<6.0$ vs. $7.0-7.9$ & $\leq 6.5$ vs. "based on local guidelines" & $\begin{array}{l}<6.0 \text { (action if }>6.5) \text { vs. } \\
\text { planned separation of } 1.5\end{array}$ \\
\hline $\begin{array}{l}\text { Protocol for glycemic control } \\
\quad \text { (I vs. S)* }\end{array}$ & $\begin{array}{l}\text { Multiple drugs in both } \\
\text { arms }\end{array}$ & $\begin{array}{l}\text { Multiple drugs added to gliclizide vs. } \\
\text { multiple drugs with no gliclizide }\end{array}$ & Multiple drugs in both arms \\
\hline $\begin{array}{l}\text { Management of other risk } \\
\text { factors }\end{array}$ & $\begin{array}{l}\text { Embedded blood pressure } \\
\text { and lipid trials }\end{array}$ & Embedded blood pressure trial & $\begin{array}{l}\text { Protocol for intensive } \\
\text { treatment in both arms }\end{array}$ \\
\hline \multicolumn{4}{|l|}{ On-study characteristics } \\
\hline \multicolumn{4}{|l|}{ On insulin at study end (\%) } \\
\hline$(\mathrm{I}$ vs. S)* & 77 vs. $55^{*}$ & 40 vs. 24 & 89 vs. 0.74 \\
\hline \multicolumn{4}{|l|}{ Weight changes (kg) } \\
\hline Intensive glycemic control arm & +3.5 & -0.1 & +7.8 \\
\hline \multicolumn{4}{|l|}{$\begin{array}{l}\text { Severe hypoglycemia } \\
\quad \text { (participants with one or more } \\
\text { episodes during study) (\%) }\end{array}$} \\
\hline Intensive glycemic control arm & 16.2 & 2.7 & 21.2 \\
\hline Standard glycemic control arm & 5.1 & 1.5 & 9.9 \\
\hline \multicolumn{4}{|l|}{ Outcomes } \\
\hline Definition of primary outcome & $\begin{array}{l}\text { Nonfatal MI, nonfatal } \\
\text { stroke, CVD death }\end{array}$ & $\begin{array}{l}\text { Microvascular plus macrovascular } \\
\text { (nonfatal MI, nonfatal stroke, CVD } \\
\text { death) outcomes }\end{array}$ & $\begin{array}{l}\text { Nonfatal MI, nonfatal stroke, } \\
\text { CVD death, } \\
\text { hospitalization for heart } \\
\text { failure, revascularization }\end{array}$ \\
\hline $\begin{array}{l}\text { HR for primary outcome } \\
\qquad(95 \% \mathrm{CI})\end{array}$ & $0.90(0.78-1.04)$ & $\begin{array}{l}0.9(0.82-0.98) \\
\text { macrovascular } 0.94(0.84-1.06)\end{array}$ & $0.88(0.74-1.05)$ \\
\hline $\begin{array}{l}\text { HR for mortality findings } \\
\quad(95 \% \mathrm{CI})\end{array}$ & $1.22(1.01-1.46)$ & $0.93(0.83-1.06)$ & $1.07(0.81-1.42)$ \\
\hline
\end{tabular}

*Insulin rates for ACCORD are for any use during the study. I, intensive glycemic control; S, standard glycemic control. Abridged from ref. 52.

(1.41 vs. 1.14\%/year, hazard ratio 1.22 , 95\% CI 1.01-1.46), with a similar increase in cardiovascular deaths. The primary outcome of ACCORD (MI, stroke, or cardiovascular death) was lower in the intensive glycemic control group due to a reduction in nonfatal $\mathrm{MI}$, although this finding was not statistically significant when the study was terminated (68). Of note, prespecified subset analyses showed that participants with no previous CVD event and those who had a baseline AlC $<8 \%$ had a statistically significant reduction in the primary CVD outcome, although overall mortality was not reduced in these groups.
The cause of excess deaths in the intensive group of the ACCORD has been difficult to pinpoint (and is discussed in some detail in a 2009 ADA position statement [52]). However, exploratory analyses of the mortality findings of ACCORD (evaluating variables including weight gain, use of any specific drug or drug combination, and hypoglycemia) were reportedly unable to identify a clear explanation for the excess mortality in the intensive arm. At the 69th Scientific Sessions of the American Diabetes Association, the ACCORD investigators presented additional analyses showing no increase in mortality in participants who achieved AlC levels $<7 \%$ or in those who lowered their AlC quickly after trial enrollment. In fact, the converse was observed: those at highest risk for mortality were participants in the intensive arm with the highest AlC levels.

The ADVANCE study randomized participants to a strategy of intensive glycemic control (with primary therapy being the sulfonylurea gliclizide and additional medications as needed to achieve a target $\mathrm{AlC}$ of $\leq 6.5 \%$ ) or to standard therapy (in which any medication but gliclizide could be used and the glycemic target was according to "local guidelines"). ADVANCE participants 
were slightly older than those in ACCORD and VADT and had similar high CVD risk. However, they had an average duration of diabetes that was 2 years shorter, lower baseline AlC (median $7.2 \%$ ), and almost no use of insulin at enrollment. The median AlC levels achieved in the intensive and standard arms were 6.3 and $7.0 \%$, respectively, and maximal separation between the arms took several years to achieve. Use of other drugs that favorably impact CVD risk (aspirin, statins, and angiotensin enzyme inhibitors) was lower in ADVANCE than in ACCORD or VADT.

The primary outcome of ADVANCE was a combination of microvascular events (nephropathy and retinopathy) and major adverse cardiovascular events (MI, stroke, and cardiovascular death). Intensive glycemic control significantly reduced the primary end point, although this was due to a significant reduction in the microvascular outcome, primarily development of macroalbuminuria, with no significant reduction in the macrovascular outcome. There was no difference in overall or cardiovascular mortality between the intensive compared with the standard glycemic control arms (63).

VADT randomized participants with type 2 diabetes uncontrolled on insulin or maximal dose oral agents (median entry AlC $9.4 \%$ ) to a strategy of intensive glycemic control (goal AlC $<6.0 \%$ ) or standard glycemic control, with a planned AlC separation of at least $1.5 \%$. Medication treatment algorithms were used to achieve the specified glycemic goals, with a goal of using similar medications in both groups. Median A1C levels of 6.9 and $8.4 \%$ were achieved in the intensive and standard arms, respectively, within the 1 st year of the study. Other CVD risk factors were treated aggressively and equally in both groups.

The primary outcome of VADT was a composite of CVD events. The cumulative primary outcome was nonsignificantly lower in the intensive arm. There were more CVD deaths in the intensive arm than in the standard arm, but the difference was not statistically significant (60). Post hoc subgroup analyses suggested that duration of diabetes interacted with randomization such that participants with duration of diabetes less than about 12 years appeared to have a CVD benefit of intensive glycemic control while those with longer duration of disease prior to study entry had a neutral or even adverse effect of intensive glycemic control. Other exploratory analyses suggested that severe hypoglycemia within the past 90 days was a strong predictor of the primary outcome and of CVD mortality (69).

All three of these trials were carried out in participants with established diabetes (mean duration 8-11 years) and either known CVD or multiple risk factors suggesting the presence of established atherosclerosis. Subset analyses of the three trials suggested a significant benefit of intensive glycemic control on CVD in participants with shorter duration of diabetes, lower AlC at entry, and/or absence of known CVD. The DCCT-EDIC study and the long-term follow-up of the UKPDS cohort both suggest that intensive glycemic control initiated soon after diagnosis of diabetes in patients with a lower level of CVD risk may impart long-term protection from CVD events. As is the case with microvascular complications, it may be that glycemic control plays a greater role before macrovascular disease is well developed and minimal or no role when it is advanced. Consistent with this concept, data from an ancillary study of VADT demonstrated that intensive glycemic control was quite effective in reducing CVD events in individuals with less atherosclerosis at baseline (assessed by coronary calcium) but not in people with more extensive baseline atherosclerosis (70).

The benefits of intensive glycemic control on microvascular and neuropathic complications are well established for both type 1 and type 2 diabetes. ADVANCE and VADT have added to that evidence base by demonstrating a significant reduction in the risk of new or worsening albuminuria with intensive glycemic control. The lack of significant reduction in CVD events with intensive glycemic control in ACCORD, ADVANCE, and VADT should not lead clinicians to abandon the general target of an AlC $<7.0 \%$ and thereby discount the benefit of good control on serious and debilitating microvascular complications.

The evidence for a cardiovascular benefit of intensive glycemic control primarily rests on long-term follow-up of study cohorts treated early in the course of type 1 and type 2 diabetes as well as subset analyses of ACCORD, ADVANCE, and VADT. A recent group-level metaanalysis of the three trials suggests that glucose lowering has a modest (9\%) but statistically significant reduction in major CVD outcomes, primarily nonfatal MI, with no significant increase in mortality.
A prespecified subgroup analysis suggested that major CVD outcome reduction occurred in patients without known CVD at baseline (HR 0.84 [95\% CI 0.740.94]) (71). Conversely, the mortality findings in ACCORD and subgroup analyses of VADT suggest that the potential risks of very intensive glycemic control may outweigh its benefits in some patients, such as those with very long duration of diabetes, known history of severe hypoglycemia, advanced atherosclerosis, and advanced age/frailty. Certainly, providers should be vigilant in preventing severe hypoglycemia in patients with advanced disease and should not aggressively attempt to achieve near-normal AlC levels in patients in whom such a target cannot be reasonably easily and safely achieved.

Recommended glycemic goals for nonpregnant adults are shown in Table 11. The recommendations are based on those for AlC values, with listed blood glucose levels that appear to correlate with achievement of an AlC of $<7 \%$. The issue of pre- versus postprandial SMBG targets is complex (72). Elevated postchallenge (2-h OGTT) glucose values have been associated with increased cardiovascular risk independent of FPG in some epidemiological studies. In diabetic subjects, some surrogate measures of vascular pathology, such as endothelial dysfunction, are negatively affected by postprandial hyperglycemia (73). It is clear that postprandial hyperglycemia, like preprandial hyperglycemia, contributes to elevated AIC levels, with its relative contribution being higher at $\mathrm{AlC}$ levels that are closer to $7 \%$. However, outcome studies have clearly shown AIC to be the primary predictor of complications, and landmark glycemic control trials such as the DCCT and UKPDS relied overwhelmingly on preprandial SMBG. Additionally, a randomized controlled trial in patients with known CVD found no CVD benefit of insulin regimens targeting postprandial glucose compared with those targeting preprandial glucose (74). For individuals who have premeal glucose values within target but A1C values above target, a reasonable recommendation for postprandial testing and targets is monitoring postprandial plasma glucose (PPG) 1-2 h after the start of the meal and treatment aimed at reducing PPG values to $<180 \mathrm{mg} / \mathrm{dl}$ to help lower AlC.

As noted above, less stringent treatment goals may be appropriate for adults with limited life expectancies or advanced 
Table 11 -Summary of glycemic recommendations for non-pregnant adults with diabetes

AlC

Preprandial capillary plasma glucose

Peak postprandial capillary plasma glucose ${ }^{\dagger}$

Key concepts in setting glycemic goals:

- $\mathrm{AlC}$ is the primary target for glycemic control

- Goals should be individualized based on:

- duration of diabetes

- age/life expectancy

- comorbid conditions

- known CVD or advanced microvascular complications

- hypoglycemia unawareness

- individual patient considerations

- More or less stringent glycemic goals may be appropriate for individual patients

Postprandial glucose may be targeted if AlC goals are not met despite reaching preprandial glucose goals

* Referenced to a nondiabetic range of $4.0-6.0 \%$ using a DCCT-based assay. $†$ Postprandial glucose measurements should be made $1-2 \mathrm{~h}$ after the beginning of the meal, generally peak levels in patients with diabetes.

vascular disease. Glycemic goals for children are provided in VII.A.1.a. Glycemic control. Severe or frequent hypoglycemia is an absolute indication for the modification of treatment regimens, including setting higher glycemic goals.

Regarding goals for glycemic control for women with GDM, recommendations from the Fifth International WorkshopConference on Gestational Diabetes (75) are to target maternal capillary glucose concentrations of:

- Preprandial $\leq 95 \mathrm{mg} / \mathrm{dl}$ (5.3 mmol/l) and either

- 1 -h postmeal $\leq 140 \mathrm{mg} / \mathrm{dl}$ (7.8 $\mathrm{mmol} / \mathrm{l}$ )

or

- 2-h postmeal $\leq 120 \mathrm{mg} / \mathrm{dl}$ (6.7 $\mathrm{mmol} / \mathrm{l}$ )

For women with preexisting type 1 or type 2 diabetes who become pregnant, a recent consensus statement (76) recommends the following as optimal glycemic goals, if they can be achieved without excessive hypoglycemia:

- premeal, bedtime, and overnight glucose 60-99 mg/dl (3.3-5.4 mmol/l)

- peak postprandial glucose 100-129 $\mathrm{mg} / \mathrm{dl}(5.4-7.1 \mathrm{mmol} / \mathrm{l})$

- $\mathrm{AlC}<6.0 \%$

\section{Approach to treatment}

a. Therapy for type 1 diabetes. The DCCT clearly showed that intensive insulin therapy (three or more injections per day of insulin or continuous subcutane- ous insulin infusion [CSII] or insulin pump therapy) was a key part of improved glycemia and better outcomes $(53,66)$. At the time of the study, therapy ter microvascular outcomes, intensive insulin therapy was associated with a high rate in severe hypoglycemia (62 episodes per 100 patient-years of therapy). Since the time of the DCCT, a number of rapidacting and long-acting insulin analogs have been developed. These analogs are associated with less hypoglycemia with equal AlC lowering in type 1 diabetes $(77,78)$.

Recommended therapy for type 1 diabetes therefore consists of the following components: 1) use of multiple dose insulin injections (3-4 injections per day of basal and prandial insulin) or CSII therapy; 2) matching of prandial insulin to carbohydrate intake, premeal blood glucose, and anticipated activity; and 3) for many patients (especially if hypoglycemia is a problem), use of insulin analogs. There are excellent reviews available that guide the initiation and management of insulin therapy to achieve desired glycemic goals $(3,77,79)$.

Because of the increased frequency of other autoimmune diseases in type 1 diabetes, screening for thyroid dysfunction, vitamin B12 deficiency, or celiac disease should be considered based on signs and symptoms. Periodic screening in the absence of symptoms has been recommended, but the effectiveness and optimal frequency are unclear. was carried out with short- and intermediate-acting human insulins. Despite bet- b. Therapy for type 2 diabetes. The ADA and the European Association for the Study of Diabetes (EASD) published a consensus statement on the approach to management of hyperglycemia in individuals with type 2 diabetes (80) and a subsequent update (81). Highlights of this approach include: intervention at the time of diagnosis with metformin in combination with lifestyle changes (MNT and exercise) and continuing timely augmentation of therapy with additional agents (including early initiation of insulin therapy) as a means of achieving and maintaining recommended levels of glycemic control (i.e., AlC $<7 \%$ for most patients). The overall objective is to achieve and maintain glycemic control and to change interventions when therapeutic goals are not being met.

The algorithm took into account the evidence for AlC lowering of the individual interventions, their additive effects, and their expense. The precise drugs used and their exact sequence may not be as important as achieving and maintaining glycemic targets safely. Medications not included in the consensus algorithm, owing to less glucose-lowering effectiveness, limited clinical data, and/or relative expense, still may be appropriate choices for individual patients to achieve glycemic goals. Initiation of insulin at the time of diagnosis is recommended for individuals presenting with weight loss or other severe hyperglycemic symptoms or signs.

\section{Medical nutrition therapy}

\section{General recommendations}

- Individuals who have pre-diabetes or diabetes should receive individualized MNT as needed to achieve treatment goals, preferably provided by a registered dietitian familiar with the components of diabetes MNT. (A)

- Because it can result in cost savings and improved outcomes (B), MNT should be covered by insurance and other payors (E).

\section{Energy balance, overweight, and obesity}

- In overweight and obese insulinresistant individuals, modest weight loss has been shown to reduce insulin resistance. Thus, weight loss is recommended for all overweight or obese individuals who have or are at risk for diabetes. (A)

- For weight loss, either low-carbohydrate or low-fat calorie-restricted diets 
may be effective in the short-term (up to 1 year). (A)

- For patients on low-carbohydrate diets, monitor lipid profiles, renal function, and protein intake (in those with nephropathy) and adjust hypoglycemic therapy as needed. (E)

- Physical activity and behavior modification are important components of weight loss programs and are most helpful in maintenance of weight loss. (B)

\section{Primary prevention of diabetes}

- Among individuals at high risk for developing type 2 diabetes, structured programs emphasizing lifestyle changes that include moderate weight loss (7\% body weight) and regular physical activity (150 min/week) with dietary strategies including reduced calories and reduced intake of dietary fat can reduce the risk for developing diabetes and are therefore recommended. (A)

- Individuals at high risk for type 2 diabetes should be encouraged to achieve the U.S. Department of Agriculture (USDA) recommendation for dietary fiber (14 g fiber/1,000 kcal) and foods containing whole grains (one-half of grain intake). (B)

\section{Dietary fat intake in diabetes management}

- Saturated fat intake should be $<7 \%$ of total calories. (A)

- Reducing intake of trans fat lowers LDL cholesterol and increases HDL cholesterol (A); therefore intake of trans fat should be minimized (E).

\section{Carbohydrate intake in diabetes management}

- Monitoring carbohydrate intake, whether by carbohydrate counting, exchanges, or experience-based estimation, remains a key strategy in achieving glycemic control. (A)

- For individuals with diabetes, use of the glycemic index and glycemic load may provide a modest additional benefit for glycemic control over that observed when total carbohydrate is considered alone. (B)

\section{Other nutrition recommendations}

- Sugar alcohols and nonnutritive sweeteners are safe when consumed within the acceptable daily intake levels established by the Food and Drug Administration (FDA). (A)
- If adults with diabetes choose to use alcohol, daily intake should be limited to a moderate amount (one drink per day or less for adult women and two drinks per day or less for adult men). (E)

- Routine supplementation with antioxidants, such as vitamins $E$ and $C$ and carotene, is not advised because of lack of evidence of efficacy and concern related to long-term safety. (A)

- Benefit from chromium supplementation in people with diabetes or obesity has not been conclusively demonstrated and therefore cannot be recommended. (C)

- Individualized meal planning should include optimization of food choices to meet recommended dietary allowances (RDAs)/dietary reference intakes (DRIs) for all micronutrients. (E)

MNT is an integral component of diabetes prevention, management, and selfmanagement education. In addition to its role in preventing and controlling diabetes, ADA recognizes the importance of nutrition as an essential component of an overall healthy lifestyle. A full review of the evidence regarding nutrition in preventing and controlling diabetes and its complications and additional nutritionrelated recommendations can be found in the ADA position statement, Nutrition Recommendations and Interventions for Diabetes, published in 2006 and updated for 2008 (82). Achieving nutritionrelated goals requires a coordinated team effort that includes the active involvement of the person with pre-diabetes or diabetes. Because of the complexity of nutrition issues, it is recommended that a registered dietitian who is knowledgeable and skilled in implementing nutrition therapy into diabetes management and education be the team member who provides MNT.

Clinical trials/outcome studies of MNT have reported decreases in AlC at 3-6 months ranging from 0.25 to $2.9 \%$ with higher reductions seen in type 2 diabetes of shorter duration. Multiple studies have demonstrated sustained improvements in AlC at 12 months and longer when a registered dietitian provided follow-up visits ranging from monthly to three sessions per year (8390). Meta-analyses of studies in nondiabetic, free-living subjects report that MNT reduces LDL cholesterol by $15-25 \mathrm{mg} / \mathrm{dl}$ (91) or by up to $16 \%$ (92), while clinical trials support a role for lifestyle modification in treating hypertension $(92,93)$.
Because of the effects of obesity on insulin resistance, weight loss is an important therapeutic objective for overweight or obese individuals with prediabetes or diabetes (94). Short-term studies have demonstrated that moderate weight loss ( $5 \%$ of body weight) in subjects with type 2 diabetes is associated with decreased insulin resistance, improved measures of glycemia and lipemia, and reduced blood pressure (95); longerterm studies ( $\geq 52$ weeks) showed mixed effects on AlC in adults with type 2 diabetes (96-99), and results were confounded by pharmacologic weight loss therapy. A systematic review of 80 weight loss studies of $\geq 1$ year duration demonstrated that moderate weight loss achieved through diet alone, diet and exercise, and meal replacements can be achieved and maintained over the long term (4.8-8\% weight loss at 12 months [100]). The multifactorial intensive lifestyle intervention used in the DPP, which included reduced intake of fat and calories, led to weight loss averaging $7 \%$ at 6 months and maintenance of $5 \%$ weight loss at 3 years, associated with a 58\% reduction in incidence of type 2 diabetes (11). Look AHEAD (Action for Health in Diabetes) is a large clinical trial designed to determine whether long-term weight loss will improve glycemia and prevent cardiovascular events in subjects with type 2 diabetes. One-year results of the intensive lifestyle intervention in this trial show an average of $8.6 \%$ weight loss, significant reduction of $\mathrm{AlC}$, and reduction in several CVD risk factors (101). When completed, the Look AHEAD study should provide insight into the effects of long-term weight loss on important clinical outcomes.

The optimal macronutrient distribution of weight loss diets has not been established. Although low-fat diets have traditionally been promoted for weight loss, several randomized controlled trials found that subjects on low-carbohydrate diets ( $<130 \mathrm{~g}$ /day of carbohydrate) lost more weight at 6 months than subjects on low-fat diets $(102,103)$; however, at 1 year, the difference in weight loss between the low-carbohydrate and low-fat diets was not significant and weight loss was modest with both diets. Another study of overweight women randomized to one of four diets showed significantly more weight loss at 12 months with the Atkins low-carbohydrate diet than with higher-carbohydrate diets (104). Changes in serum triglyceride and HDL 
cholesterol were more favorable with the low-carbohydrate diets. In one study, those subjects with type 2 diabetes demonstrated a greater decrease in $\mathrm{AlC}$ with a low-carbohydrate diet than with a low-fat diet (103). A recent meta-analysis showed that at 6 months, low-carbohydrate diets were associated with greater improvements in triglyceride and HDL cholesterol concentrations than low-fat diets; however, LDL cholesterol was significantly higher with the low-carbohydrate diets (105). In a 2-year dietary intervention study, Mediterranean and low-carbohydrate diets were found to be effective and safe alternatives to a low-fat diet for weight reduction in moderately obese participants (99).

The RDA for digestible carbohydrate is $130 \mathrm{~g} /$ day and is based on providing adequate glucose as the required fuel for the central nervous system without reliance on glucose production from ingested protein or fat. Although brain fuel needs can be met on lower-carbohydrate diets, long-term metabolic effects of very-lowcarbohydrate diets are unclear, and such diets eliminate many foods that are important sources of energy, fiber, vitamins, and minerals that are important in dietary palatability (106).

Although numerous studies have attempted to identify the optimal mix of macronutrients for meal plans of people with diabetes, it is unlikely that one such combination of macronutrients exists. The best mix of carbohydrate, protein, and fat appears to vary depending on individual circumstances. For those individuals seeking guidance as to macronutrient distribution in healthy adults, DRIs may be helpful (106). It must be clearly recognized that regardless of the macronutrient mix, the total caloric intake must be appropriate to the weight management goal. Further, individualization of the macronutrient composition will depend on the metabolic status of the patient (e.g., lipid profile and renal function) and/or food preferences. Plantbased diets (vegan or vegetarian) that are well planned and nutritionally adequate have also been shown to improve metabolic control $(107,108)$.

The primary goal with respect to dietary fat in individuals with diabetes is to limit saturated fatty acids, trans fatty acids, and cholesterol intake so as to reduce risk for CVD. Saturated and trans fatty acids are the principal dietary determinants of plasma LDL cholesterol. There is a lack of evidence on the effects of specific fatty acids on people with diabetes; therefore, the recommended goals are consistent with those for individuals with CVD $(92,109)$.

The FDA has approved five nonnutritive sweeteners for use in the U.S.: acesulfame potassium, aspartame, neotame, saccharin, and sucralose. Before being allowed on the market, all underwent rigorous scrutiny and were shown to be safe when consumed by the public, including people with diabetes and women during pregnancy. Reduced calorie sweeteners approved by the FDA include sugar alcohols (polyols) such as erythritol, isomalt, lactitol, maltitol, mannitol, sorbitol, xylitol, tagatose, and hydrogenated starch hydrolysates. The use of sugar alcohols appears to be safe; however, they may cause diarrhea, especially in children. Stevia (Rebaudioside A) has been designated by the FDA as being generally recognized as safe (GRAS).

\section{Reimbursement for MNT}

MNT, when delivered by a registered dietitian according to nutrition practice guidelines, is reimbursed as part of the Medicare program as overseen by the Centers for Medicare and Medicaid Services (www. cms.hhs.gov/ medicalnutritiontherapy).

\section{E. Bariatric surgery}

\section{Recommendations}

- Bariatric surgery should be considered for adults with BMI $>35 \mathrm{~kg} / \mathrm{m}^{2}$ and type 2 diabetes, especially if the diabetes or associated comorbidities are difficult to control with lifestyle and pharmacologic therapy. (B)

- Patients with type 2 diabetes who have undergone bariatric surgery need lifelong lifestyle support and medical monitoring. (E)

- Although small trials have shown glycemic benefit of bariatric surgery in patients with type 2 diabetes and BMI of 30-35 $\mathrm{kg} / \mathrm{m}^{2}$, there is currently insufficient evidence to generally recommend surgery in patients with BMI $<35 \mathrm{~kg} / \mathrm{m}^{2}$ outside of a research protocol. (E)

- The long-term benefits, cost-effectiveness, and risks of bariatric surgery in individuals with type 2 diabetes should be studied in well-designed, randomized controlled trials with optimal medical and lifestyle therapy as the comparator. (E)

Gastric reduction surgery, either gastric banding or procedures that involve by- passing or transposing sections of the small intestine, when part of a comprehensive team approach, can be an effective weight loss treatment for severe obesity, and national guidelines support its consideration for people with type 2 diabetes who have BMI $>35 \mathrm{~kg} / \mathrm{m}^{2}$. Bariatric surgery has been shown to lead to near or complete normalization of glycemia in $~ 55-95 \%$ of patients with type 2 diabetes, depending on the surgical procedure. A meta-analysis of studies of bariatric surgery reported that $78 \%$ of individuals with type 2 diabetes had complete "resolution" of diabetes (normalization of blood glucose levels in the absence of medications) and that the resolution rates were sustained in studies that had follow-up exceeding 2 years (110). Resolution rates are lower with procedures that only constrict the stomach and higher with those that bypass portions of the small intestine. Additionally, there is a suggestion that intestinal bypass procedures may have glycemic effects that are independent of their effects on weight.

A recent randomized controlled trial compared adjustable gastric banding to the "best available" medical and lifestyle therapy in subjects with type 2 diabetes diagnosed $<2$ years before randomization and with BMI $30-40 \mathrm{~kg} / \mathrm{m}^{2}$ (111). In this trial, $73 \%$ of surgically treated patients achieved "remission" of their diabetes, compared with $13 \%$ of those treated medically. The latter group lost only $1.7 \%$ of body weight, suggesting that their therapy was not optimal. Overall the trial had 60 subjects, and only 13 had a BMI $<35$ $\mathrm{kg} / \mathrm{m}^{2}$, making it difficult to generalize these results to diabetic patients who are less severely obese or with longer duration of diabetes.

Bariatric surgery is costly in the short term and has some risks. Rates of morbidity and mortality directly related to the surgery have been reduced considerably in recent years, with 30-day mortality rates now $0.28 \%$, similar to those of laparoscopic cholecystectomy (112). Longer-term concerns include vitamin and mineral deficiencies, osteoporosis, and rare but often severe hypoglycemia from insulin hypersecretion. Cohort studies attempting to match subjects suggest that the procedure may reduce longer-term mortality rates (113), and it is reasonable to postulate that there may be recouping of costs over the long term. However, studies of the mechanisms of glycemic improvement, long-term benefits and risks, and cost-effectiveness of bariatric surgery in individuals with type 2 diabetes will require 
well-designed, randomized clinical trials with optimal medical and lifestyle therapy of diabetes and cardiovascular risk factors as the comparators.

\section{F. Diabetes self-management education}

\section{Recommendations}

- People with diabetes should receive DSME according to national standards when their diabetes is diagnosed and as needed thereafter. (B)

- Effective self-management and quality of life are the key outcomes of DSME and should be measured and monitored as part of care. (C)

- DSME should address psychosocial issues, since emotional well-being is associated with positive diabetes outcomes. (C)

- Because DSME can result in costsavings and improved outcomes (B), DSME should be reimbursed by thirdparty payors. (E)

DSME is an essential element of diabetes care (114-120), and national standards for DSME (121) are based on evidence for its benefits. Education helps people with diabetes initiate effective selfmanagement and cope with diabetes when they are first diagnosed. Ongoing DSME and support also help people with diabetes maintain effective selfmanagement throughout a lifetime of diabetes as they face new challenges and as treatment advances become available. DSME helps patients optimize metabolic control, prevent and manage complications, and maximize quality of life in a cost-effective manner (122).

DSME is the on-going process of facilitating the knowledge, skill, and ability necessary for diabetes self-care (121). This process incorporates the needs, goals, and life experiences of the person with diabetes. The overall objectives of DSME are to support informed decisionmaking, self-care behaviors, problemsolving, and active collaboration with the health care team and to improve clinical outcomes, health status, and quality of life in a cost-effective manner (121).

Current best practice of DSME is a skills-based approach that focuses on helping those with diabetes make informed self-management choices. DSME has changed from a didactic approach focusing on providing information, to a more theoretically based empowerment model that focuses on helping those with diabetes make informed self-management decisions. Care of diabetes has shifted to an approach that is more patient centered and places the person with diabetes at the center of the care model working in collaboration with health care professionals. Patient-centered care is respectful of and responsive to individual patient preferences, needs, and values and ensures that patient values guide all decision making (123).

1. Evidence for the benefits of DSME Multiple studies have found that DSME is associated with improved diabetes knowledge and self-care behavior (115); improved clinical outcomes such as lower AlC $(116,117,119,120,124)$, lower selfreported weight (115), improved quality of life $(118,125)$, and healthy coping (126); and lower costs (127). Better outcomes were reported for DSME interventions that were longer and included follow-up support $(115,128-131)$, that were culturally (132) and age appropriate $(133,134)$ and tailored to individual needs and preferences (114), and that addressed psychosocial issues $(114,115$, $119,135)$. Both individual and group approaches have been found effective (136138). There is growing evidence for the role of community health workers and peer (139) and lay leaders (140) in delivering DSME and support in addition to the core team (141).

Diabetes education is associated with increased use of primary and preventive services and lower use of acute, inpatient hospital services (127). Patients who participate in diabetes education are more likely to follow best practice treatment recommendations, particularly among the medicare population, and to have lower Medicare and commercial claim costs (142).

\section{National Standards for DSME}

The National Standards for DSME are designed to define quality diabetes selfmanagement education and to assist diabetes educators in a variety of settings to provide evidence-based education (121). The standards, most recently revised in 2007, are reviewed and updated every 5 years by a task force representing key organizations involved in the field of diabetes education and care.

\section{Reimbursement for DSME}

DSME, when provided by a program that meets ADA recognition standards, is reimbursed as part of the Medicare program overseen by the Centers for Medicare and Medicaid Services (www.cms.hhs.gov/ DiabetesSelfManagement).

\section{G. Physical activity}

\section{Recommendations}

- People with diabetes should be advised to perform at least $150 \mathrm{~min} /$ week of moderate-intensity aerobic physical activity (50-70\% of maximum heart rate). (A)

- In the absence of contraindications, people with type 2 diabetes should be encouraged to perform resistance training three times per week. (A)

ADA technical reviews on exercise in patients with diabetes, currently being updated, have summarized the value of exercise in the diabetes management plan $(143,144)$. Regular exercise has been shown to improve blood glucose control, reduce cardiovascular risk factors, contribute to weight loss, and improve well being. Furthermore, regular exercise may prevent type 2 diabetes in high-risk individuals (11-13). Structured exercise interventions of at least 8 weeks' duration have been shown to lower AlC by an average of $0.66 \%$ in people with type 2 diabetes, even with no significant change in BMI (145). Higher levels of exercise intensity are associated with greater improvements in AlC and fitness (146).

\section{Frequency and type of exercise}

The U.S. Department of Health and Human Services' Physical Activity Guidelines for Americans (147) suggest that adults over age 18 years perform $150 \mathrm{~min} /$ week of moderate-intensity or $75 \mathrm{~min} /$ week of vigorous aerobic physical activity or an equivalent combination of the two. In addition, the guidelines suggest that adults also do muscle-strengthening activities that involve all major muscle groups two or more days per week. The guidelines suggest that adults over age 65 years, or those with disabilities, follow the adult guidelines if possible or (if this is not possible) be as physically active as they are able. Studies included in the metaanalysis of effects of exercise interventions on glycemic control (145) had a mean number of sessions per week of 3.4, with a mean of $49 \mathrm{~min} / \mathrm{session}$. The DPP lifestyle intervention, which included 150 min/week of moderate intensity exercise, had a beneficial effect on glycemia in those with pre-diabetes. Therefore, it seems reasonable to recommend that peo- 
ple with diabetes try to follow the physical activity guidelines for the general population.

Progressive resistance exercise improves insulin sensitivity in older men with type 2 diabetes to the same or even to a greater extent as aerobic exercise (148) Clinical trials have provided strong evidence for the AlC-lowering value of resistance training in older adults with type 2 diabetes $(149,150)$ and for an additive benefit of combined aerobic and resistance exercise in adults with type 2 diabetes (151).

\section{Evaluation of the diabetic patient before recommending an exercise program}

Prior guidelines have suggested that before recommending a program of physical activity, the provider should assess patients with multiple cardiovascular risk factors for coronary artery disease (CAD). As further discussed in VI.A.5. Coronary heart disease screening and treatment, the area of screening asymptomatic diabetic patients for CAD remains unclear, and a recent $\mathrm{ADA}$ consensus statement on this issue concluded that routine screening is not recommended (152). Providers should use clinical judgment in this area. Certainly, high-risk patients should be encouraged to start with short periods of low-intensity exercise and to increase the intensity and duration slowly.

Providers should assess patients for conditions that might contraindicate certain types of exercise or predispose to injury, such as uncontrolled hypertension, severe autonomic neuropathy, severe peripheral neuropathy or history of foot lesions, and unstable proliferative retinopathy. The patient's age and previous physical activity level should be considered.

\section{Exercise in the presence of nonoptimal glycemic control}

a. Hyperglycemia. When people with type 1 diabetes are deprived of insulin for 12-48 $\mathrm{h}$ and are ketotic, exercise can worsen hyperglycemia and ketosis (153); therefore, vigorous activity should be avoided in the presence of ketosis. However, it is not necessary to postpone exercise simply based on hyperglycemia, provided the patient feels well and urine and/or blood ketones are negative.

b. Hypoglycemia. In individuals taking insulin and/or insulin secretagogues, physical activity can cause hypoglycemia if medication dose or carbohydrate con- sumption is not altered. For individuals on these therapies, added carbohydrate should be ingested if pre-exercise glucose levels are $<100 \mathrm{mg} / \mathrm{dl}(5.6 \mathrm{mmol} / \mathrm{l})$ $(154,155)$. Hypoglycemia is rare in diabetic individuals who are not treated with insulin or insulin secretagogues, and no preventive measures for hypoglycemia are usually advised in these cases.

\section{Exercise in the presence of specific long-term complications of diabetes} a. Retinopathy. In the presence of proliferative diabetic retinopathy (PDR) or severe non-proliferative diabetic retinopathy (NPDR), vigorous aerobic or resistance exercise may be contraindicated because of the risk of triggering vitreous hemorrhage or retinal detachment (156). b. Peripheral neuropathy. Decreased pain sensation in the extremities results in increased risk of skin breakdown and infection and of Charcot joint destruction. Prior recommendations have advised non-weight-bearing exercise for patients with severe peripheral neuropathy. Studies have shown that moderate-intensity walking may not lead to increased risk of foot ulcers or reulceration in those with peripheral neuropathy (157). All individuals with peripheral neuropathy should wear proper footwear and examine their feet daily for early detection of lesions. Anyone with a foot injury or open sore should be restricted to non-weightbearing activities.

c. Autonomic neuropathy. Autonomic neuropathy can increase the risk of exercise-induced injury or adverse events through decreased cardiac responsiveness to exercise, postural hypotension, impaired thermoregulation, impaired night vision due to impaired papillary reaction, and unpredictable carbohydrate delivery from gastroparesis predisposing to hypoglycemia (158). Autonomic neuropathy is also strongly associated with CVD in people with diabetes $(159,160)$. People with diabetic autonomic neuropathy should undergo cardiac investigation before beginning physical activity more intense than that to which they are accustomed.

d. Albuminuria and nephropathy. Physical activity can acutely increase urinary protein excretion. However, there is no evidence that vigorous exercise increases the rate of progression of diabetic kidney disease and likely no need for any specific exercise restrictions for people with diabetic kidney disease (161).

\section{H. Psychosocial assessment and care}

\section{Recommendations}

- Assessment of psychological and social situation should be included as an ongoing part of the medical management of diabetes. (E)

- Psychosocial screening and follow-up should include, but is not limited to, attitudes about the illness, expectations for medical management and outcomes, affect/mood, general and diabetes-related quality of life, resources (financial, social, and emotional), and psychiatric history. (E)

- Screen for psychosocial problems such as depression and diabetes-related distress, anxiety, eating disorders, and cognitive impairment when selfmanagement is poor. (C)

Psychological and social problems can impair the ability of the individual (162164) or the family to carry out diabetes care tasks and therefore compromise health status. There are opportunities for the clinician to assess psychosocial status in a timely and efficient manner so that referral for appropriate services can be accomplished.

Key opportunities for screening of psychosocial status occur at diagnosis, during regularly scheduled management visits, during hospitalizations, at discovery of complications, or when problems with glucose control, quality of life, or adherence are identified. Patients are likely to exhibit psychological vulnerability at diagnosis and when their medical status changes, i.e., the end of the honeymoon period, when the need for intensified treatment is evident, and when complications are discovered (164).

Issues known to impact selfmanagement and health outcomes include but are not limited to: attitudes about the illness, expectations for medical management and outcomes, affect $/ \mathrm{mood}$, general and diabetes-related quality of life, diabetes-related distress (165), resources (financial, social, and emotional) (166), and psychiatric history $(167,168)$. Screening tools are available for a number of these areas (135). Indications for referral to a mental health specialist familiar with diabetes management may include gross noncompliance with medical regimen (by self or others) (168), depression with the possibility of self-harm $(169,170)$, debilitating anxiety (alone or with depression), indications of an eating disorder, or cognitive functioning that 
significantly impairs judgment. It is preferable to incorporate psychological assessment and treatment into routine care rather than waiting for identification of a specific problem or deterioration in psychological status (135). Although the clinician may not feel qualified to treat psychological problems, using the patient-provider relationship as a foundation for further treatment can increase the likelihood that the patient will accept referral for other services. It is important to establish that emotional well-being is part of diabetes management.

\section{When treatment goals are not met}

For a variety of reasons, some people with diabetes and their health care providers do not achieve the desired goals of treatment (Table 11). Rethinking the treatment regimen may require assessment of barriers including income, health literacy, diabetes distress, depression, and competing demands, including those related to family responsibilities and dynamics. Other strategies may include culturally appropriate and enhanced DSME, comanagement with a diabetes team, referral to a medical social worker for assistance with insurance coverage, or change in pharmacological therapy. Initiation of or increase in SMBG, utilization of CGM, frequent contact with the patient, or referral to a mental health professional or physician with special expertise in diabetes may be useful. Providing patients with an algorithm for self-titration of insulin doses based on SMBG results may be helpful for type 2 patients who take insulin (171).

\section{J. Intercurrent illness}

The stress of illness, trauma, and/or surgery frequently aggravates glycemic control and may precipitate diabetic ketoacidosis (DKA) or nonketotic hyperosmolar state, life-threatening conditions that require immediate medical care to prevent complications and death (172). Any condition leading to deterioration in glycemic control necessitates more frequent monitoring of blood glucose and (in ketosis-prone patients) urine or blood ketones. Marked hyperglycemia requires temporary adjustment of the treatment program and, if accompanied by ketosis, vomiting, or alteration in level of consciousness, immediate interaction with the diabetes care team. The patient treated with noninsulin therapies or MNT alone may temporarily require insulin. Adequate fluid and caloric intake must be as- sured. Infection or dehydration are more likely to necessitate hospitalization of the person with diabetes than the person without diabetes.

The hospitalized patient should be treated by a physician with expertise in the management of diabetes. For further information on management of patients with hyperglycemia in the hospital, see VIII.A. Diabetes care in the hospital. For further information on management of DKA or nonketotic hyperosmolar state, refer to the ADA consensus statement on hyperglycemic crises (173).

\section{K. Hypoglycemia}

\section{Recommendations}

- Glucose (15-20 g) is the preferred treatment for the conscious individual with hypoglycemia, although any form of carbohydrate that contains glucose may be used. If SMBG 15 min after treatment shows continued hypoglycemia, the treatment should be repeated. Once SMBG glucose returns to normal, the individual should consume a meal or snack to prevent recurrence of hypoglycemia. (E)

- Glucagon should be prescribed for all individuals at significant risk of severe hypoglycemia, and caregivers or family members of these individuals should be instructed in its administration. Glucagon administration is not limited to health care professionals. (E)

- Individuals with hypoglycemia unawareness or one or more episodes of severe hypoglycemia should be advised to raise their glycemic targets to strictly avoid further hypoglycemia for at least several weeks to partially reverse hypoglycemia unawareness and reduce risk of future episodes. (B)

Hypoglycemia is the leading limiting factor in the glycemic management of type 1 and insulin-treated type 2 diabetes (174). Treatment of hypoglycemia (PG $<70 \mathrm{mg}$ / dl) requires ingestion of glucose- or carbohydrate-containing foods. The acute glycemic response correlates better with the glucose content than with the carbohydrate content of the food. Although pure glucose is the preferred treatment, any form of carbohydrate that contains glucose will raise blood glucose. Added fat may retard and then prolong the acute glycemic response (175). Ongoing activity of insulin or insulin secretagogues may lead to recurrence of hypoglycemia unless further food is ingested after recovery.
Severe hypoglycemia (where the individual requires the assistance of another person and cannot be treated with oral carbohydrate due to confusion or unconsciousness) should be treated using emergency glucagon kits, which require a prescription. Those in close contact with or who have custodial care of people with hypoglycemia-prone diabetes (family members, roommates, school personnel, child care providers, correctional institution staff, or coworkers) should be instructed in use of such kits. An individual does not need to be a health care professional to safely administer glucagon. Care should be taken to ensure that unexpired glucagon kits are available.

Prevention of hypoglycemia is a critical component of diabetes management. Teaching people with diabetes to balance insulin use, carbohydrate intake, and exercise is a necessary but not always sufficient strategy. In type 1 diabetes and severely insulin-deficient type 2 diabetes, the syndrome of hypoglycemia unawareness, or hypoglycemia-associated autonomic failure, can severely compromise stringent diabetes control and quality of life. The deficient counter-regulatory hormone release and autonomic responses in this syndrome are both risk factors for and are caused by hypoglycemia. A corollary to this "vicious cycle" is that several weeks of avoidance of hypoglycemia has been demonstrated to improve counterregulation and awareness to some extent in many patients $(174,176,177)$. Hence, patients with one or more episodes of severe hypoglycemia may benefit from at least short-term relaxation of glycemic targets.

\section{Immunization}

\section{Recommendations}

- Annually provide an influenza vaccine to all diabetic patients $\geq 6$ months of age. (C)

- Administer pneumococcal polysaccharide vaccine to all diabetic patients $\geq 2$ years of age. A one-time revaccination is recommended for individuals $>64$ years of age previously immunized when they were $<65$ years of age if the vaccine was administered $>5$ years ago. Other indications for repeat vaccination include nephrotic syndrome, chronic renal disease, and other immunocompromised states, such as after transplantation. (C) 
Influenza and pneumonia are common, preventable infectious diseases associated with high mortality and morbidity in the elderly and in people with chronic diseases. Though there are limited studies reporting the morbidity and mortality of influenza and pneumococcal pneumonia specifically in people with diabetes, observational studies of patients with a variety of chronic illnesses, including diabetes, show that these conditions are associated with an increase in hospitalizations for influenza and its complications. People with diabetes may be at increased risk of the bacteremic form of pneumococcal infection and have been reported to have a high risk of nosocomial bacteremia, which has a mortality rate as high as 50\% (178).

Safe and effective vaccines are available that can greatly reduce the risk of serious complications from these diseases $(179,180)$. In a case-control series, influenza vaccine was shown to reduce diabetes-related hospital admission by as much as 79\% during flu epidemics (179). There is sufficient evidence to support that people with diabetes have appropriate serologic and clinical responses to these vaccinations. The Centers for Disease Control and Prevention's Advisory Committee on Immunization Practices recommends influenza and pneumococcal vaccines for all individuals with diabetes (http://www.cdc.gov/vaccines/recs/). For a complete discussion on the prevention of influenza and pneumococcal disease in people with diabetes, consult the technical review and position statement on this subject $(178,181)$.

\section{PREVENTION AND MANAGEMENT OF DIABETES COMPLICATIONS}

\section{A. Cardiovascular disease}

CVD is the major cause of morbidity and mortality for individuals with diabetes and the largest contributor to the direct and indirect costs of diabetes. The common conditions coexisting with type 2 diabetes (e.g., hypertension and dyslipidemia) are clear risk factors for CVD, and diabetes itself confers independent risk. Numerous studies have shown the efficacy of controlling individual cardiovascular risk factors in preventing or slowing CVD in people with diabetes. Large benefits are seen when multiple risk factors are addressed globally $(182,183)$. Risk for coronary heart disease and CVD in general can be estimated using multivariable risk factor approaches, and such a strategy may be desirable to undertake in adult patients prior to instituting preventive therapy.

\section{Hypertension/blood pressure control}

\section{Recommendations}

\section{Screening and diagnosis}

- Blood pressure should be measured at every routine diabetes visit. Patients found to have systolic blood pressure $\geq 130 \mathrm{mmHg}$ or diastolic blood pressure $\geq 80 \mathrm{mmHg}$ should have blood pressure confirmed on a separate day. Repeat systolic blood pressure $\geq 130$ $\mathrm{mmHg}$ or diastolic blood pressure $\geq 80$ mmHg confirms a diagnosis of hypertension. (C)

\section{Goals}

- Patients with diabetes should be treated to a systolic blood pressure $<130$ mmHg. (C)

- Patients with diabetes should be treated to a diastolic blood pressure $<80$ mmHg. (B)

\section{Treatment}

- Patients with a systolic blood pressure 130-139 mmHg or a diastolic blood pressure $80-89 \mathrm{mmHg}$ may be given lifestyle therapy alone for a maximum of 3 months, and then if targets are not achieved, patients should be treated with the addition of pharmacological agents. (E)

- Patients with more severe hypertension (systolic blood pressure $\geq 140 \mathrm{mmHg}$ or diastolic blood pressure $\geq 90$ $\mathrm{mmHg}$ ) at diagnosis or follow-up should receive pharmacologic therapy in addition to lifestyle therapy. (A)

- Lifestyle therapy for hypertension consists of weight loss if overweight, DASH-style dietary pattern including reducing sodium and increasing potassium intake, moderation of alcohol intake, and increased physical activity. (B)

- Pharmacologic therapy for patients with diabetes and hypertension should be paired with a regimen that includes either an ACE inhibitor or an angiotensin II receptor blocker (ARB). If one class is not tolerated, the other should be substituted. If needed to achieve blood pressure targets, a thiazide diuretic should be added to those with an estimated glomerular filtration rate (GFR) (see below) $\geq 30 \mathrm{ml} \cdot \mathrm{min} / 1.73$ $\mathrm{m}^{2}$ and a loop diuretic for those with an estimated GFR $<30 \mathrm{ml} \cdot \mathrm{min} / 1.73 \mathrm{~m}^{2}$. (C)

- Multiple drug therapy (two or more agents at maximal doses) is generally required to achieve blood pressure targets. (B)

- If ACE inhibitors, ARBs, or diuretics are used, kidney function and serum potassium levels should be closely monitored. (E)

- In pregnant patients with diabetes and chronic hypertension, blood pressure target goals of 110-129/65-79 mmHg are suggested in the interest of longterm maternal health and minimizing impaired fetal growth. ACE inhibitors and $A R B s$ are contraindicated during pregnancy.

Hypertension is a common comorbidity of diabetes that affects the majority of patients, with prevalence depending on type of diabetes, age, obesity, and ethnicity. Hypertension is a major risk factor for both CVD and microvascular complications. In type 1 diabetes, hypertension is often the result of underlying nephropathy, while in type 2 diabetes it usually coexists with other cardiometabolic risk factors.

a. Screening and diagnosis. Measurement of blood pressure in the office should be done by a trained individual and should follow the guidelines established for nondiabetic individuals: measurement in the seated position, with feet on the floor and arm supported at heart level, after 5 min of rest. Cuff size should be appropriate for the upper arm circumference. Elevated values should be confirmed on a separate day. Because of the clear synergistic risks of hypertension and diabetes, the diagnostic cutoff for a diagnosis of hypertension is lower in people with diabetes (blood pressure $\geq 130 / 80$ $\mathrm{mmHg}$ ) than in those without diabetes (blood pressure $\geq 140 / 90 \mathrm{mmHg}$ ) (184).

Home blood pressure self-monitoring and 24-h ambulatory blood pressure monitoring may provide additional evidence of "white coat" and masked hypertension and other discrepancies between office and "true" blood pressure, and studies in nondiabetic populations show that home measurements may correlate better with CVD risk than office measurements $(185,186)$. However, the preponderance of the clear evidence of benefits of treatment of hypertension in people with diabetes is based on office measurements. 
b. Treatment goals. Randomized clinical trials have demonstrated the benefit (reduction of coronary heart disease [CHD] events, stroke, and nephropathy) of lowering blood pressure to $<140 \mathrm{mmHg}$ systolic and $<80 \mathrm{mmHg}$ diastolic in individuals with diabetes (184,187-189). Epidemiologic analyses show that blood pressure $>115 / 75 \mathrm{mmHg}$ is associated with increased cardiovascular event rates and mortality in individuals with diabetes $(184,190,191)$. Therefore, a target blood pressure goal of $<130 / 80 \mathrm{mmHg}$ is reasonable if it can be achieved safely. The ongoing ACCORD trial is designed to determine whether blood pressure lowering to systolic blood pressure $<120 \mathrm{mmHg}$ provides greater cardiovascular protection than a systolic blood pressure level of $<140 \mathrm{mmHg}$ in patients with type 2 diabetes (192).

c. Treatment strategies. Although there are no well-controlled studies of diet and exercise in the treatment of hypertension in individuals with diabetes, the Dietary Approaches to Stop Hypertension (DASH) study in nondiabetic individuals has shown antihypertensive effects similar to those of pharmacologic monotherapy. Lifestyle therapy consists of reducing sodium intake (to $<1,500 \mathrm{mg} /$ day) and excess body weight; increasing consumption of fruits, vegetables (8-10 servings/day), and low-fat dairy products (2-3 servings/day); avoiding excessive alcohol consumption (no more than two servings per day in men and no more than one serving per day in women); and increasing activity levels $(184,193)$. These nonpharmacological strategies may also positively affect glycemia and lipid control. Their effects on cardiovascular events have not been established. An initial trial of nonpharmacologic therapy may be reasonable in diabetic individuals with mild hypertension (systolic 130-139 mmHg or diastolic 80-89 $\mathrm{mmHg}$ ). If the blood pressure is $\geq 140$ $\mathrm{mmHg}$ systolic and/or $\geq 90 \mathrm{mmHg}$ diastolic at the time of diagnosis, pharmacologic therapy should be initiated along with nonpharmacologic therapy (184).

Lowering of blood pressure with regimens based on a variety of antihypertensive drugs, including ACE inhibitors, ARBs, $\beta$-blockers, diuretics, and calcium channel blockers, has been shown to be effective in reducing cardiovascular events. Several studies suggested that ACE inhibitors may be superior to dihydropyridine calcium channel blockers in reducing cardiovascular events (194196). However, a variety of other studies have shown no specific advantage to ACE inhibitors as initial treatment of hypertension in the general hypertensive population, but rather an advantage on cardiovascular outcomes of initial therapy with low-dose thiazide diuretics $(184,197,198)$.

In people with diabetes, inhibitors of the renin-angiotensin system (RAS) may have unique advantages for initial or early therapy of hypertension. In a nonhypertension trial of high-risk individuals including a large subset with diabetes, an ACE inhibitor reduced CVD outcomes (199). In patients with congestive heart failure (CHF), including diabetic subgroups, ARBs have been shown to reduce major CVD outcomes (200-203), and in type 2 patients with significant nephropathy, ARBs were superior to calcium channel blockers for reducing heart failure (204-206). Though evidence for distinct advantages of RAS inhibitors on CVD outcomes in diabetes remains conflicting $(187,207)$, the high CVD risks associated with diabetes, and the high prevalence of undiagnosed CVD, may still favor recommendations for their use as first-line hypertension therapy in people with diabetes (184). Recently, the blood pressure arm of the ADVANCE trial demonstrated that routine administration of a fixed combination of the ACE inhibitor perindopril and the diuretic indapamide significantly reduced combined microvascular and macrovascular outcomes, as well as CVD and total mortality. The improved outcomes also could have been due to lower achieved blood pressure in the perindopril-indapamide arm (208). In addition, the ACCOMPLISH (Avoiding Cardiovascular Events in Combination Therapy in Patients Living with Systolic Hypertension) trial showed a decrease in morbidity and mortality in those receiving benazapril and amlodipine versus benazapril and hydrochlorothiazide. The compelling benefits of RAS inhibitors in diabetic patients with albuminuria or renal insufficiency provide additional rationale for use of these agents (see below, VI.B. Nephropathy screening and treatment).

An important caveat is that most patients with hypertension require multidrug therapy to reach treatment goals, especially diabetic patients whose targets are lower. Many patients will require three or more drugs to reach target goals (184). If blood pressure is refractory to optimal doses of at least three antihypertensive agents of different classifications, one of which should be a diuretic, clinicians should consider an evaluation for secondary forms of hypertension.

During pregnancy in diabetic women with chronic hypertension, target blood pressure goals of 110-129 mmHg systolic and 65-79 $\mathrm{mmHg}$ diastolic are reasonable, as they contribute to long-term maternal health. Lower blood pressure levels may be associated with impaired fetal growth. During pregnancy, treatment with ACE inhibitors and ARBs is contraindicated, since they can cause fetal damage. Antihypertensive drugs known to be effective and safe in pregnancy include methyldopa, labetalol, diltiazem, clonidine, and prazosin. Chronic diuretic use during pregnancy has been associated with restricted maternal plasma volume, which might reduce uteroplacental perfusion (209).

\section{Dyslipidemia/lipid management}

\section{Recommendations}

\section{Screening}

- In most adult patients, measure fasting lipid profile at least annually. In adults with low-risk lipid values (LDL cholesterol <100 mg/dl, HDL cholesterol $>50 \mathrm{mg} / \mathrm{dl}$, and triglycerides <150 $\mathrm{mg} / \mathrm{dl}$ ), lipid assessments may be repeated every 2 years. (E)

\section{Treatment recommendations and goals}

- Lifestyle modification focusing on the reduction of saturated fat, trans fat, and cholesterol intake; increase of n-3 fatty acids, viscous fiber, and plant stanols/sterols; weight loss (if indicated); and increased physical activity should be recommended to improve the lipid profile in patients with diabetes. (A)

- Statin therapy should be added to lifestyle therapy, regardless of baseline lipid levels, for diabetic patients:

- with overt CVD. (A)

- without CVD who are over the age of 40 years and have one or more other CVD risk factors. (A)

- For patients at lower risk than described above (e.g., without overt CVD and under the age of 40 years), statin therapy should be considered in addition to lifestyle therapy if LDL cholesterol remains $>100 \mathrm{mg} / \mathrm{dl}$ or in those with multiple CVD risk factors. (E)

- In individuals without overt CVD, the 
Table 12-Reduction in 10-year risk of major CVD endpoints (CHD death/non-fatal MI) in major statin trials, or sub-studies of major trials, in diabetic subjects $(\mathrm{N}=16,032)$

\begin{tabular}{|c|c|c|c|c|c|c|}
\hline$\underline{\text { Study (ref.) }}$ & $\begin{array}{c}\text { CVD } \\
\text { prevention } \\
\end{array}$ & Statin dose and comparator & Risk reduction & $\begin{array}{c}\text { Relative risk } \\
\text { reduction }\end{array}$ & $\begin{array}{c}\text { Absolute risk } \\
\text { reduction }\end{array}$ & $\begin{array}{c}\text { LDL } \\
\text { cholesterol } \\
\text { reduction }\end{array}$ \\
\hline 4S-DM (211) & $2^{\circ}$ & Simvastatin $20-40 \mathrm{mg}$ vs. placebo & 85.7 to $43.2 \%(50 \%)$ & $42.5 \%$ & 186 to $119 \mathrm{mg} / \mathrm{dl}$ & $36 \%$ \\
\hline ASPEN $2^{\circ}(216)$ & $2^{\circ}$ & Atorvastatin 10 mg vs. placebo & 39.5 to $24.5 \%(34 \%)$ & $12.7 \%$ & 112 to $79 \mathrm{mg} / \mathrm{dl}$ & $29 \%$ \\
\hline HPS-DM (212) & $2^{\circ}$ & Simvastatin 40 mg vs. placebo & 43.8 to $36.3 \%(17 \%)$ & $7.5 \%$ & 123 to $84 \mathrm{mg} / \mathrm{dl}$ & $31 \%$ \\
\hline CARE-DM (213) & $2^{\circ}$ & Pravastatin 40 mg vs. placebo & 40.8 to $35.4 \%(13 \%)$ & $5.4 \%$ & 136 to $99 \mathrm{mg} / \mathrm{dl}$ & $27 \%$ \\
\hline TNT-DM (214) & $2^{\circ}$ & Atorvastatin $80 \mathrm{mg}$ vs. $10 \mathrm{mg}$ & 26.3 to $21.6 \%(18 \%)$ & $4.7 \%$ & 99 to $77 \mathrm{mg} / \mathrm{dl}$ & $22 \%$ \\
\hline HPS-DM (212) & $1^{\circ}$ & Simvastatin $40 \mathrm{mg}$ vs. placebo & 17.5 to $11.5 \%(34 \%)$ & $6.0 \%$ & 124 to $86 \mathrm{mg} / \mathrm{dl}$ & $31 \%$ \\
\hline CARDS (234) & $1^{\circ}$ & Atorvastatin $10 \mathrm{mg}$ vs. placebo & 11.5 to $7.5 \%(35 \%)$ & $4.0 \%$ & 118 to $71 \mathrm{mg} / \mathrm{dl}$ & $40 \%$ \\
\hline ASPEN $1^{\circ}(216)$ & $1^{\circ}$ & Atorvastatin $10 \mathrm{mg}$ vs. placebo & 9.8 to $7.9 \%(19 \%)$ & $1.9 \%$ & 114 to $80 \mathrm{mg} / \mathrm{dl}$ & $30 \%$ \\
\hline ASCOT-DM (215) & $1^{\circ}$ & Atorvastatin $10 \mathrm{mg}$ vs. placebo & 11.1 to $10.2 \%(8 \%)$ & $0.9 \%$ & 125 to $82 \mathrm{mg} / \mathrm{dl}$ & $34 \%$ \\
\hline
\end{tabular}

Studies were of differing lengths (3.3-5.4 years) and used somewhat different outcomes, but all reported rates of CVD death and non-fatal MI. In this tabulation, results of the statin on 10-year risk of major CVD endpoints (CHD death/non-fatal MI) are listed for comparison between studies. Correlation between 10-year CVD risk of the control group and the absolute risk reduction with statin therapy is highly significant $(P=0.0007)$. Analyses provided by $\mathrm{Craig}$ Williams, PharmD, Oregon Health \& Science University, 2007.

primary goal is an LDL cholesterol $<100 \mathrm{mg} / \mathrm{dl}(2.6 \mathrm{mmol} / \mathrm{l})$. (A)

- In individuals with overt CVD, a lower LDL cholesterol goal of $<70 \mathrm{mg} / \mathrm{dl}(1.8$ $\mathrm{mmol} / \mathrm{l})$, using a high dose of a statin, is an option. (B)

- If drug-treated patients do not reach the above targets on maximal tolerated statin therapy, a reduction in LDL cholesterol of $\sim 30-40 \%$ from baseline is an alternative therapeutic goal. (A)

- Triglycerides levels $<150$ mg/dl (1.7 $\mathrm{mmol} / \mathrm{l}$ ) and HDL cholesterol $>40$ $\mathrm{mg} / \mathrm{dl}(1.0 \mathrm{mmol} / \mathrm{l})$ in men and $>50$ $\mathrm{mg} / \mathrm{dl}(1.3 \mathrm{mmol} / \mathrm{l})$ in women, are desirable. However, LDL cholesteroltargeted statin therapy remains the preferred strategy. (C)

- If targets are not reached on maximally tolerated doses of statins, combination therapy using statins and other lipidlowering agents may be considered to achieve lipid targets but has not been evaluated in outcome studies for either CVD outcomes or safety. (E)

- Statin therapy is contraindicated in pregnancy. (E)

\footnotetext{
a. Evidence for benefits of lipid-lowering therapy. Patients with type 2 diabetes have an increased prevalence of lipid abnormalities, contributing to their high risk of CVD. Over the past decade or more, multiple clinical trials have demonstrated significant effects of pharmacologic (primarily statin) therapy on CVD outcomes in subjects with CHD and for primary CVD prevention (210). Analyses of diabetic subgroups of larger trials (211-215) and trials specifically in subjects with diabetes $(216,217)$ showed sig-

Table 13-Summary of recommendations for glycemic, blood pressure, and lipid control for adults with diabetes

\begin{tabular}{ll}
\hline AlC & $<7.0 \% *$ \\
Blood pressure & $<130 / 80 \mathrm{mmHg}$ \\
Lipids & \\
$\quad$ LDL cholesterol & $<100 \mathrm{mg} / \mathrm{dl}(<2.6$ \\
& $\mathrm{mmol} / \mathrm{l}) \dagger$
\end{tabular}

*Referenced to a nondiabetic range of $4.0-6.0 \%$ using a DCCT-based assay. †In individuals with overt CVD, a lower LDL cholesterol goal of $<70$ $\mathrm{mg} / \mathrm{dl}(1.8 \mathrm{mmol} / \mathrm{l})$, using a high dose of a statin, is an option.
}

nificant primary and secondary prevention of CVD events with and without CHD deaths in diabetic populations. As shown in Table 12, and similar to findings in nondiabetic subjects, reduction in "hard" CVD outcomes (CHD death and nonfatal MI) can be more clearly seen in diabetic subjects with high baseline CVD risk (known CVD and/or very high LDL cholesterol levels), but overall the benefits of statin therapy in people with diabetes at moderate or high risk for CVD are convincing.

Low levels of HDL cholesterol, often associated with elevated triglyceride levels, are the most prevalent pattern of dyslipidemia in people with type 2 diabetes. However, the evidence base for drugs that target these lipid fractions is significantly less robust than that for statin therapy (217). Nicotinic acid has been shown to reduce CVD outcomes (218), although the study was done in a nondiabetic cohort. Gemfibrozil has been shown to decrease rates of CVD events in subjects without diabetes $(219,220)$ and in a di- abetic subgroup of a larger trial (219). However, in a large trial specific to diabetic patients, fenofibrate failed to reduce overall cardiovascular outcomes (221).

b. Dyslipidemia treatment and target lipid levels. For most patients with diabetes, the first priority of dyslipidemia therapy (unless severe hypertriglyceridemia is the immediate issue) is to lower LDL cholesterol to a target goal of $<100$ $\mathrm{mg} / \mathrm{dl}(2.60 \mathrm{mmol} / \mathrm{l})(222)$. Lifestyle intervention, including MNT, increased physical activity, weight loss, and smoking cessation, may allow some patients to reach lipid goals. Nutrition intervention should be tailored according to each patient's age, type of diabetes, pharmacological treatment, lipid levels, and other medical conditions and should focus on the reduction of saturated fat, cholesterol, and trans unsaturated fat intake and increases in n-3 fatty acids, viscous fiber (such as in oats, legumes, citrus), and plant stanols/sterols. Glycemic control can also beneficially modify plasma lipid levels, particularly in patients with very high triglycerides and poor glycemic control.

In those with clinical CVD or who are over age 40 years and have CVD risk factors, pharmacological treatment should be added to lifestyle therapy regardless of baseline lipid levels. Statins are the drugs of choice for lowering LDL cholesterol.

In patients other than those described above, statin treatment should be considered if there is an inadequate LDL cholesterol response to lifestyle modifications and improved glucose control or if the patient has increased cardiovascular risk 
(e.g., multiple cardiovascular risk factors or long duration of diabetes). Very little clinical trial evidence exists for type 2 diabetic patients under the age of 40 years and for type 1 diabetic patients of any age. In the Heart Protection Study (lower age limit 40 years), the subgroup of 600 patients with type 1 diabetes had a proportionately similar reduction in risk as patients with type 2 diabetes although not statistically significant (212). Although the data are not definitive, consideration should be given to lipid-lowering goals for type 1 diabetic patients similar to those for type 2 diabetic patients, particularly if other cardiovascular risk factors are present.

c. Alternative LDL cholesterol goals. Virtually all trials of statins and CVD outcome have tested specific doses of statins against placebo, other doses of statin, or other statins, rather than aiming for specific LDL cholesterol goals (223). As can be seen in Table 10, placebo-controlled trials generally achieved LDL cholesterol reductions of $30-40 \%$ from baseline. Hence, LDL cholesterol lowering of this magnitude is an acceptable outcome for patients who cannot reach LDL cholesterol goals due to severe baseline elevations in LDL cholesterol and/or intolerance of maximal, or any, statin doses. Additionally, for those with baseline LDL cholesterol minimally $>100 \mathrm{mg} /$ $\mathrm{dl}$, prescribing statin therapy to lower LDL cholesterol to $~ 30-40 \%$ from baseline is probably more effective than prescribing just enough to get LDL cholesterol slightly $<100 \mathrm{mg} / \mathrm{dl}$.

Recent clinical trials in high-risk patients, such as those with acute coronary syndromes or previous cardiovascular events (224-226), have demonstrated that more aggressive therapy with high doses of statins to achieve an LDL cholesterol of $<70 \mathrm{mg} / \mathrm{dl}$ led to a significant reduction in further events. Therefore, a reduction in LDL cholesterol to a goal of $<70 \mathrm{mg} / \mathrm{dl}$ is an option in very-high-risk diabetic patients with overt CVD (227).

In individual patients, LDL cholesterol lowering with statins is highly variable, and this variable response is poorly understood (228). Reduction of CVD events with statins correlates very closely with LDL cholesterol lowering (229). When maximally tolerated doses of statins fail to significantly lower LDL cholesterol $(<30 \%$ reduction from patients baseline), the primary aim of combination therapy should be to achieve additional LDL cholesterol lowering. Niacin, fenofi- brate, ezetimibe, and bile acid sequestrants all offer additional LDL cholesterol lowering. The evidence that combination therapy provides a significant increment in CVD risk reduction over statin therapy alone is still elusive.

d. Treatment of other lipoprotein fractions or targets. Severe hypertriglyceridemia may warrant immediate therapy of this abnormality with lifestyle and usually pharmacologic therapy (fibric acid derivative or niacin) to reduce the risk of acute pancreatitis. In the absence of severe hypertriglyceridemia, therapy targeting HDL cholesterol or triglycerides has intuitive appeal but lacks the evidence base of statin therapy (186). If the HDL cholesterol is $<40 \mathrm{mg} / \mathrm{dl}$ and the LDL cholesterol is $100-129 \mathrm{mg} / \mathrm{dl}$, gemfibrozil or niacin might be used, especially if a patient is intolerant to statins. Niacin is the most effective drug for raising HDL cholesterol. It can significantly increase blood glucose at high doses, but recent studies demonstrate that at modest doses (7502,000 mg/day), significant improvements in LDL cholesterol, HDL cholesterol, and triglyceride levels are accompanied by only modest changes in glucose that are generally amenable to adjustment of diabetes therapy $(230,231)$.

Combination therapy with a statin and a fibrate or a statin and niacin may be efficacious for treatment of all three lipid fractions, but this combination is associated with an increased risk for abnormal transaminase levels, myositis, or rhabdomyolysis. The risk of rhabdomyolysis is higher with higher doses of statins and with renal insufficiency and seems to be lower when statins are combined with fenofibrate than gemfibrozil (232). Several ongoing trials may provide much-needed evidence for the effects of combination therapy on cardiovascular outcomes.

In 2008, a consensus panel convened by ADA and the American College of Cardiology (ACC) recommended a greater focus on non-HDL cholesterol and apo lipoprotein B (apo B) in patients who are likely to have small LDL particles, such as people with diabetes (233). The consensus panel suggested that for statin-treated patients in whom the LDL cholesterol goal would be $<70 \mathrm{mg} / \mathrm{dl}$ (non-HDL cholesterol $<100 \mathrm{mg} / \mathrm{dl}$ ), apo B should be measured and treated to $<80 \mathrm{mg} / \mathrm{dl}$. For patients on statins with an LDL cholesterol goal of $<100 \mathrm{mg} / \mathrm{dl}$ (non-HDL cholesterol $<130 \mathrm{mg} / \mathrm{dl}$ ), apo B should be measured and treated to $<90 \mathrm{mg} / \mathrm{dl}$.

For a summary of recommendations for glycemic, blood pressure, and lipid control for adults with diabetes, see Table 13.

\section{Antiplatelet agents}

\section{Recommendations}

- Consider aspirin therapy (75-162 mg/ day) as a primary prevention strategy in those with type 1 or type 2 diabetes at increased cardiovascular risk (10-year risk $>10 \%$ ). This includes most men $>50$ years of age or women $>60$ years of age who have at least one additional major risk factor (family history of CVD, hypertension, smoking, dyslipidemia, or albuminuria). (C)

- There is not sufficient evidence to recommend aspirin for primary prevention in lower risk individuals, such as men $<50$ years of age or women $<60$ years of age without other major risk factors. For patients in these age-groups with multiple other risk factors, clinical judgment is required. (C)

- Use aspirin therapy (75-162 mg/day) as a secondary prevention strategy in those with diabetes with a history of CVD. (A)

- For patients with CVD and documented aspirin allergy, clopidogrel (75 mg/day) should be used. (B)

- Combination therapy with ASA (75$162 \mathrm{mg} /$ day) and clopidogrel (75 mg/ day) is reasonable for up to a year after an acute coronary syndrome. (B)

ADA and the American Heart Association (AHA) have, in the past, jointly recommended that low-dose aspirin therapy be used as a primary prevention strategy in those with diabetes at increased cardiovascular risk, including those who are over 40 years of age or those with additional risk factors (family history of CVD, hypertension, smoking, dyslipidemia, or albuminuria) (235). These recommendations were derived from several older trials that included small numbers of patients with diabetes.

Aspirin has been shown to be effective in reducing cardiovascular morbidity and mortality in high-risk patients with previous MI or stroke (secondary prevention). Its net benefit in primary prevention among patients with no previous cardiovascular events is more controversial, both for patients with and without a history of diabetes (236). The U.S. Preventive Services Task Force recently updated its evidence base and recommendations about aspirin use for primary pre- 
vention $(237,238)$. The Task Force recommended encouraging aspirin use in men 45-79 and women 55-79 years of age and not encouraging aspirin use in younger adults and did not differentiate based on the presence or absence of diabetes.

Two recent randomized controlled trials of aspirin specifically in patients with diabetes failed to show a significant reduction in CVD end points, raising further questions about the efficacy of aspirin for primary prevention in people with diabetes $(239,240)$. In 2009, ADA AHA, and ACC convened a group of experts to review and synthesize the available evidence and use this information to create an updated recommendation. Their report, including analyses in addition to those described below, will be published in early 2010.

The ATT (Anti-Thrombotic Trialists') collaborators recently published an individual patient-level meta-analysis of the six large trials of aspirin for primary prevention in the general population (236). These trials collectively enrolled over 95,000 participants, including almost 4,000 with diabetes. Overall, they found that aspirin reduced the risk of vascular events by $12 \%$ (RR 0.88 [95\% CI 0.82 $0.94])$. The largest reduction was for nonfatal MI (0.77 [0.67-0.89]). Aspirin had little effect on CHD death (0.95 [0.781.15]) or total stroke (0.95 [0.85-1.06]). The net effect on total stroke reflected a relative reduction in risk of ischemic stroke $(-14 \%)$ and a relative increased risk of hemorrhagic stroke $(+32 \%)$. There was some evidence of a difference in aspirin effect by sex. Aspirin reduced CHD events in men (0.77 [0.67-0.89]) but not in women (0.95 [0.77-1.17]). Conversely, aspirin had no effect on stroke in men (1.01 [0.74-1.39]) but reduced stroke in women $(0.77$ [0.590.99]). These potential differences in effect by sex were of borderline statistical significance, were affected strongly by the results of one trial, and cannot be considered definitive. Notably, sex differences in aspirin's effects have not been observed in studies of secondary prevention (236). In the six trials examined by the ATT collaborators, the effect of aspirin on major vascular events was similar for patients with and without diabetes (0.88 [0.67-1.15] and $0.87[0.79-0.96]$, respectively). The CI was wider for those with diabetes because of their smaller number.
Based on the currently available evidence, aspirin appears to have a modest effect on ischemic vascular events with the absolute decrease in events depending on the underlying CVD risk. The main adverse effects appear to be an increased risk of gastrointestinal bleeding. The excess risk may be as high as $1-5$ per 1,000 per year in real-world settings. In adults with CVD risk greater than $1 \%$ per year, the number of CVD events prevented will be similar to or greater than the number of episodes of bleeding induced, although these complications do not have equal effects on long-term health (241).

Average daily dosages used in most clinical trials involving patients with diabetes ranged from 50-650 mg but were mostly in the range of $100-325 \mathrm{mg} /$ day. There is little evidence to support any specific dose, but using the lowest possible dosage may help reduce side effects (242). Although platelets from patients with diabetes have altered function, it is unclear what, if any, impact that finding has on the required dose of aspirin for cardioprotective effects in the patient with diabetes. Many alternate pathways for platelet activation exist that are independent of thromboxane $A_{2}$ and thus not sensitive to the effects of aspirin (243). Therefore, while "aspirin resistance" appears higher in diabetic patients when measured by a variety of ex vivo and in vitro methods (platelet aggrenometry, measurement of thromboxane $B_{2}$ ), these observations alone are insufficient to empirically recommend at this time that higher doses of aspirin be used in the diabetic patient (244-246).

Aspirin use for secondary prevention continues to have a strong evidence base and is recommended. Until further evidence is available, low-dose (75-162 mg/ day) aspirin use for primary prevention is reasonable for adults with diabetes and no previous history of vascular disease who are at increased CVD risk (10-year risk of CVD events $>10 \%$ ) and who are not at increased risk for bleeding. This generally includes most men over age 50 years and women over age 60 years who also have one or more of the following major risk factors: smoking, hypertension, dyslipidemia, family history of premature CVD, and albuminuria.

Aspirin should not be recommended for those at low CVD risk (women under age 60 years and men under age 50 years with no major CVD risk factors; 10-year CVD risk $<5 \%$ ), as the low benefit is off- set by the incidence of significant bleeding. Clinical judgment should be used for those at intermediate risk (younger patients with one or risk factors or older patients with no risk factors; those with 10year CVD risk 5-10\%) until further research is available. Use of aspirin in patients under the age of 21 years is contraindicated due to the associated risk of Reye's syndrome.

Clopidogrel has been demonstrated to reduce CVD events in diabetic individuals (247). It is recommended as adjunctive therapy in the 1st year after an acute coronary syndrome or as alternative therapy in aspirin-intolerant patients.

\section{Smoking cessation}

\section{Recommendations}

- Advise all patients not to smoke. (A)

- Include smoking cessation counseling and other forms of treatment as a routine component of diabetes care. (B)

Issues of smoking and diabetes are reviewed in detail in the ADA technical review (248) and position statement (249) on this topic. A large body of evidence from epidemiological, case-control, and cohort studies provides convincing documentation of the causal link between cigarette smoking and health risks. Cigarette smoking contributes to one of every five deaths in the U.S. and is the most important modifiable cause of premature death. Much of the prior work documenting the impact of smoking on health did not separately discuss results on subsets of individuals with diabetes, suggesting that the identified risks are at least equivalent to those found in the general population. Other studies of individuals with diabetes consistently found a heightened risk of CVD and premature death among smokers. Smoking is also related to the premature development of microvascular complications of diabetes and may have a role in the development of type 2 diabetes.

A number of large randomized clinical trials have demonstrated the efficacy and cost-effectiveness of smoking cessation counseling in changing smoking behavior and reducing tobacco use. The routine and thorough assessment of tobacco use is important as a means of preventing smoking or encouraging cessation. Special considerations should include assessment of level of nicotine dependence, which is associated with difficulty in quitting and relapse $(250,251)$. 


\section{Coronary heart disease screening and treatment}

\section{Recommendations}

\section{Screening}

- In asymptomatic patients, evaluate risk factors to stratify patients by 10 -year risk, and treat risk factors accordingly. (B)

\section{Treatment}

- In patients with known CVD, ACE inhibitor (C), aspirin (A), and statin therapy (A) (if not contraindicated) should be used to reduce the risk of cardiovascular events.

- In patients with a prior MI, $\beta$-blockers should be continued for at least 2 years after the event. (B)

- Longer-term use of $\beta$-blockers in the absence of hypertension is reasonable if well tolerated, but data are lacking. (E)

- Avoid thiazolidinedione (TZD) treatment in patients with symptomatic heart failure. (C)

- Metformin may be used in patients with stable CHF if renal function is normal. It should be avoided in unstable or hospitalized patients with $\mathrm{CHF}$. (C)

Screening for CAD is reviewed in a recently updated consensus statement (93). To identify the presence of CAD in diabetic patients without clear or suggestive symptoms, a risk factor-based approach to the initial diagnostic evaluation and subsequent follow-up has intuitive appeal. However, recent studies concluded that using this approach fails to identify which patients will have silent ischemia on screening tests $(159,252)$.

Candidates for cardiac testing include those with 1) typical or atypical cardiac symptoms and 2) an abnormal resting electrocardiogram (ECG). The screening of asymptomatic patients remains controversial, especially since intensive medical therapy, indicated in diabetic patients at high risk for CVD, has an increasing evidence base for providing equal outcomes to invasive revascularization, including in diabetic patients $(253,254)$. There is also recent preliminary evidence that silent myocardial ischemia may reverse over time, adding to the controversy concerning aggressive screening strategies (255). Finally, a recent randomized observational trial demonstrated no clinical benefit to routine screening of asymptomatic patients with type 2 diabetes and normal ECGs (256). Despite abnormal myocar- dial perfusion imaging in more than one in five patients, cardiac outcomes were essentially equal (and very low) in screened versus unscreened patients. Accordingly, the overall effectiveness, especially the cost-effectiveness, of such an indiscriminate screening strategy is in question.

In all patients with diabetes, cardiovascular risk factors should be assessed at least annually. These risk factors include dyslipidemia, hypertension, smoking, a positive family history of premature coronary disease, and the presence of microor macroalbuminuria. Abnormal risk factors should be treated as described elsewhere in these guidelines. Patients at increased CHD risk should receive aspirin and a statin, and ACE inhibitor, or ARB therapy if hypertensive, unless there are contraindications to a particular drug class. While clear benefit exists for ACE inhibitor and ARB therapy in patients with nephropathy or hypertension, the benefits in patients with CVD in the absence of these conditions is less clear, especially when LDL cholesterol is concomitantly controlled $(257,258)$.

\section{B. Nephropathy screening and treatment}

\section{Recommendations}

\section{General recommendations}

- To reduce the risk or slow the progression of nephropathy, optimize glucose control. (A)

- To reduce the risk or slow the progression of nephropathy, optimize blood pressure control. (A)

\section{Screening}

- Perform an annual test to assess urine albumin excretion in type 1 diabetic patients with diabetes duration of 5 years and in all type 2 diabetic patients, starting at diagnosis. (E)

- Measure serum creatinine at least annually in all adults with diabetes regardless of the degree of urine albumin excretion. The serum creatinine should be used to estimate GFR and stage the level of chronic kidney disease (CKD), if present. (E)

\section{Treatment}

- In the treatment of the nonpregnant patient with micro- or macroalbuminuria, either ACE inhibitors or ARBs should be used. (A)

- While there are no adequate head-tohead comparisons of ACE inhibitors and ARBs, there is clinical trial support for each of the following statements:

- In patients with type 1 diabetes, hypertension, and any degree of albuminuria, ACE inhibitors have been shown to delay the progression of nephropathy. (A)

- In patients with type 2 diabetes, hypertension, and microalbuminuria, both ACE inhibitors and ARBs have been shown to delay the progression to macroalbuminuria. (A)

- In patients with type 2 diabetes, hypertension, macroalbuminuria, and renal insufficiency (serum creatinine $>1.5 \mathrm{mg} / \mathrm{dl}$ ), ARBs have been shown to delay the progression of nephropathy. (A)

- If one class is not tolerated, the other should be substituted. (E)

- Reduction of protein intake to 0.8-1.0 $\mathrm{g} \cdot \mathrm{kg}$ body $\mathrm{wt}^{-1} \cdot \mathrm{day}^{-1}$ in individuals with diabetes and the earlier stages of CKD and to $0.8 \mathrm{~g} \cdot \mathrm{kg}$ body $\mathrm{wt}^{-1} \cdot$ day $^{-1}$ in the later stages of CKD may improve measures of renal function (urine albumin excretion rate and GFR) and is recommended. (B)

- When ACE inhibitors, ARBs, or diuretics are used, monitor serum creatinine and potassium levels for the development of acute kidney disease and hyperkalemia. (E)

- Continued monitoring of urine albumin excretion to assess both response to therapy and progression of disease is recommended. (E)

- Consider referral to a physician experienced in the care of kidney disease when there is uncertainty about the etiology of kidney disease (active urine sediment, absence of retinopathy, or rapid decline in GFR), difficult management issues, or advanced kidney disease. (B)

Diabetic nephropathy occurs in 20-40\% of patients with diabetes and is the single leading cause of end-stage renal disease (ESRD). Persistent albuminuria in the range of 30-299 mg/24 h (microalbuminuria) has been shown to be the earliest stage of diabetic nephropathy in type 1 diabetes and a marker for development of nephropathy in type 2 diabetes. Microalbuminuria is also a well-established marker of increased CVD risk $(259,260)$. Patients with microalbuminuria who progress to macroalbuminuria ( $\geq 300$ $\mathrm{mg} / 24 \mathrm{~h}$ ) are likely to progress to ESRD $(261,262)$. However, a number of interventions have been demonstrated to re- 
duce the risk and slow the progression of renal disease.

Intensive diabetes management with the goal of achieving nearnormoglycemia has been shown in large prospective randomized studies to delay the onset of microalbuminuria and the progression of micro- to macroalbuminuria in patients with type 1 $(263,264)$ and type $2(57,58)$ diabetes. The UKPDS provided strong evidence that control of blood pressure can reduce the development of nephropathy (187). In addition, large prospective randomized studies in patients with type 1 diabetes have demonstrated that achievement of lower levels of systolic blood pressure $(<140 \mathrm{mmHg}$ ) resulting from treatment using ACE inhibitors provides a selective benefit over other antihypertensive drug classes in delaying the progression from micro- to macroalbuminuria and can slow the decline in GFR in patients with macroalbuminuria $(205,206,265)$. In type 2 diabetes with hypertension and normoalbuminuria, RAS inhibition has been demonstrated to delay onset of microalbuminuria (266).

In addition, ACE inhibitors have been shown to reduce major CVD outcomes (i.e., MI, stroke, and death) in patients with diabetes (199), thus further supporting the use of these agents in patients with microalbuminuria, a CVD risk factor. ARBs do not prevent microalbuminuria in normotensive patients with type 1 or type 2 diabetes $(267,268)$; however, ARBs have been shown to reduce the rate of progression from micro- to macroalbuminuria as well as ESRD in patients with type 2 diabetes (269-271). Some evidence suggests that ARBs have a smaller magnitude of rise in potassium compared with ACE inhibitors in people with nephropathy $(272,273)$. It is important to note that both ACE inhibitors and ARBs reduce loss of kidney function in people with diabetic nephropathy, above and beyond any such effect attributable to a reduction in systemic blood pressure. Combinations of drugs that block the rennin-angiotensin-aldosterone system (e.g., an ACE inhibitor plus an ARB, a mineralocorticoid antagonist, or a direct renin inhibitor) have been shown to provide additional lowering of albuminuria (274277). However, the long-term effects of such combinations on renal or cardiovascular outcomes have not yet been evaluated in clinical trials.

Other drugs, such as diuretics, calcium channel blockers, and $\beta$-blockers,
Table 14-Definitions of abnormalities in albumin excretion

\begin{tabular}{lc}
\hline Category & $\begin{array}{c}\text { Spot collection } \\
(\mu \mathrm{g} / \mathrm{mg} \\
\text { creatinine })\end{array}$ \\
\hline Normal & $<30$ \\
Microalbuminuria & $30-299$ \\
Macroalbuminuria (clinical) & $\geq 300$ \\
\hline
\end{tabular}

should be used as additional therapy to further lower blood pressure in patients already treated with ACE inhibitors or ARBs (204) or as alternate therapy in the rare individual unable to tolerate ACE inhibitors or ARBs.

Studies in patients with varying stages of nephropathy have shown that protein restriction helps slow the progression of albuminuria, GFR decline, and occurrence of ESRD (278-281). Protein restriction should be considered particularly in patients whose nephropathy seems to be progressing despite optimal glucose and blood pressure control and use of ACE inhibitor and/or ARBs (281).

\section{Assessment of albuminuria status and renal function}

Screening for microalbuminuria can be performed by measurement of the albumin-to-creatinine ratio in a random spot collection (preferred method); 24-h or timed collections are more burdensome and add little to prediction or accuracy $(282,283)$. Measurement of a spot urine for albumin only, whether by immunoassay or by using a dipstick test specific for microalbumin, without simultaneously measuring urine creatinine, is somewhat less expensive but susceptible to falsenegative and -positive determinations as a result of variation in urine concentration due to hydration and other factors.

Abnormalities of albumin excretion are defined in Table 14. Because of variability in urinary albumin excretion, two of three specimens collected within a 3- to 6-month period should be abnormal before considering a patient to have crossed one of these diagnostic thresholds. Exercise within $24 \mathrm{~h}$, infection, fever, CHF, marked hyperglycemia, and marked hypertension may elevate urinary albumin excretion over baseline values.

Information on presence of abnormal urine albumin excretion in addition to level of GFR may be used to stage CKD. The National Kidney Foundation classifi- cation (Table 15) is primarily based on GFR levels and therefore differs from other systems, in which staging is based primarily on urinary albumin excretion (284). Studies have found decreased GFR in the absence of increased urine albumin excretion in a substantial percentage of adults with diabetes $(285,286)$. Epidemiologic evidence suggests that a substantial fraction of those with CKD in the setting of diabetes have little or no detectable albuminuria (285). Serum creatinine should therefore be measured at least annually in all adults with diabetes, regardless of the degree of urine albumin excretion.

Serum creatinine should be used to estimate GFR and to stage the level of CKD, if present. Estimated GFR (eGFR) is commonly co-reported by laboratories or can be estimated using formulae such as the Modification of Diet in Renal Disease (MDRD) study equation (287). Recent reports have indicated that the MDRD is more accurate for the diagnosis and stratification of CKD in patients with diabetes than the Cockcroft-Gault formula (288). GFR calculators are available at http:// www.nkdep.nih.gov.

The role of continued annual quantitative assessment of albumin excretion after diagnosis of microalbuminuria and institution of ACE inhibitor or ARB therapy and blood pressure control is unclear. Continued surveillance can assess both response to therapy and progression of disease. Some suggest that reducing abnormal albuminuria $(>30 \mathrm{mg} / \mathrm{g}$ ) to the normal or near-normal range may improve renal and cardiovascular prognosis, but this approach has not been formally evaluated in prospective trials.

Complications of kidney disease correlate with level of kidney function. When the eGFR is less than $60 \mathrm{ml} \cdot \mathrm{min} / 1.73 \mathrm{~m}^{2}$, screening for anemia, malnutrition, and metabolic bone disease is indicated. Early vaccination against Hepatitis B is indicated in patients likely to progress to endstage kidney disease.

Consider referral to a physician experienced in the care of kidney disease when there is uncertainty about the etiology of kidney disease (active urine sediment, absence of retinopathy, or rapid decline in GFR), difficult management issues, or advanced kidney disease. The threshold for referral may vary depending on the frequency with which a provider encounters diabetic patients with significant kidney disease. Consultation with a nephrologist when stage 4 CKD develops has been 
Table 15-Stages of CKD

\begin{tabular}{llc} 
Stage & \multicolumn{1}{c}{ Description } & $\begin{array}{c}\text { GFR (ml/min per } \\
1.73 \mathrm{~m}^{2} \text { body } \\
\text { surface area) }\end{array}$ \\
\hline 1 & Kidney damage* with normal or increased GFR $_{2}$ & $\mathbf{2 9 0}$ \\
3 & Kidney damage* with mildly decreased GFR $^{2}$ & $60-89$ \\
4 & Moderately decreased GFR & $30-59$ \\
5 & Severely decreased GFR & $15-29$ \\
\hline
\end{tabular}

*Kidney damage defined as abnormalities on pathologic, urine, blood, or imaging tests. Adapted from ref. 283.

found to reduce cost, improve quality of care, and keep people off dialysis longer $(289,290)$. However, nonrenal specialists should not delay educating their patients about the progressive nature of diabetic kidney disease, the renal preservation benefits of aggressive treatment of blood pressure, blood glucose, and hyperlipidemia, and the potential need for renal replacement therapy.

\section{Retinopathy screening and treatment}

\section{Recommendations}

\section{General recommendations}

- To reduce the risk or slow the progression of retinopathy, optimize glycemic control. (A)

- To reduce the risk or slow the progression of retinopathy, optimize blood pressure control. (A)

\section{Screening}

- Adults and children aged 10 years or older with type 1 diabetes should have an initial dilated and comprehensive eye examination by an ophthalmologist or optometrist within 5 years after the onset of diabetes. (B)

- Patients with type 2 diabetes should have an initial dilated and comprehensive eye examination by an ophthalmologist or optometrist shortly after the diagnosis of diabetes. (B)

- Subsequent examinations for type 1 and type 2 diabetic patients should be repeated annually by an ophthalmologist or optometrist. Less frequent exams (every 2-3 years) may be considered following one or more normal eye exams. Examinations will be required more frequently if retinopathy is progressing. (B)

- High-quality fundus photographs can detect most clinically significant dia- betic retinopathy. Interpretation of the images should be performed by a trained eye care provider. While retinal photography may serve as a screening tool for retinopathy, it is not a substitute for a comprehensive eye exam, which should be performed at least initially and at intervals thereafter as recommended by an eye care professional. (E)

- Women with preexisting diabetes who are planning pregnancy or who have become pregnant should have a comprehensive eye examination and be counseled on the risk of development and/or progression of diabetic retinopathy. Eye examination should occur in the first trimester with close follow-up throughout pregnancy and for 1 year postpartum. (B)

\section{Treatment}

- Promptly refer patients with any level of macular edema, severe NPDR, or any PDR to an ophthalmologist who is knowledgeable and experienced in the management and treatment of diabetic retinopathy. (A)

- Laser photocoagulation therapy is indicated to reduce the risk of vision loss in patients with high-risk PDR, clinically significant macular edema, and in some cases of severe NPDR. (A)

- The presence of retinopathy is not a contraindication to aspirin therapy for cardioprotection, as this therapy does not increase the risk of retinal hemorrhage. (A)

Diabetic retinopathy is a highly specific vascular complication of both type 1 and type 2 diabetes, with prevalence strongly related to duration of diabetes. Diabetic retinopathy is the most frequent cause of new cases of blindness among adults aged 20-74 years. Glaucoma, cataracts, and other disorders of the eye occur earlier and more frequently in people with diabetes.

In addition to duration of diabetes, other factors that increase the risk of, or are associated with, retinopathy include chronic hyperglycemia (291), the presence of nephropathy (292), and hypertension (293). Intensive diabetes management with the goal of achieving near normoglycemia has been shown in large prospective randomized studies to prevent and/or delay the onset and progression of diabetic retinopathy $(53,57,58)$. Lowering blood pressure has been shown to decrease the progression of retinopathy (187). Several case series and a controlled prospective study suggest that pregnancy in type 1 diabetic patients may aggravate retinopathy $(294,295)$; laser photocoagulation surgery can minimize this risk (295).

One of the main motivations for screening for diabetic retinopathy is the established efficacy of laser photocoagulation surgery in preventing vision loss. Two large trials, the Diabetic Retinopathy Study (DRS) and the Early Treatment Diabetic Retinopathy Study (ETDRS), provide the strongest support for the therapeutic benefits of photocoagulation surgery.

The DRS (296) showed that panretinal photocoagulation surgery reduced the risk of severe vision loss from PDR from $15.9 \%$ in untreated eyes to $6.4 \%$ in treated eyes. The benefit was greatest among patients whose baseline evaluation revealed high-risk characteristics (chiefly disc neovascularization or vitreous hemorrhage). Given the risks of modest loss of visual acuity and contraction of the visual field from panretinal laser surgery, such therapy is primarily recommended for eyes with PDR approaching or having high-risk characteristics.

The ETDRS (297) established the benefit of focal laser photocoagulation surgery in eyes with macular edema, particularly those with clinically significant macular edema, with reduction of doubling of the visual angle (e.g., 20/ $50-20 / 100$ ) from $20 \%$ in untreated eyes to $8 \%$ in treated eyes. The ETDRS also verified the benefits of panretinal photocoagulation for high-risk PDR, but not for mild or moderate NPDR. In older-onset patients with severe NPDR or less-than-high-risk PDR, the risk of severe vision loss or vitrectomy was reduced $50 \%$ by early laser photocoagulation surgery at these stages. 
Laser photocoagulation surgery in both trials was beneficial in reducing the risk of further vision loss, but generally not beneficial in reversing already diminished acuity. This preventive effect and the fact that patients with PDR or macular edema may be asymptomatic provide strong support for a screening program to detect diabetic retinopathy.

As retinopathy is estimated to take at least 5 years to develop after the onset of hyperglycemia (298), patients with type 1 diabetes should have an initial dilated and comprehensive eye examination within 5 years after the onset of diabetes. Patients with type 2 diabetes who generally have had years of undiagnosed diabetes (299) and who have a significant risk of prevalent diabetic retinopathy at the time of diabetes diagnosis should have an initial dilated and comprehensive eye examination soon after diagnosis. Examinations should be performed by an ophthalmologist or optometrist who is knowledgeable and experienced in diagnosing the presence of diabetic retinopathy and is aware of its management. Subsequent examinations for type 1 and type 2 diabetic patients are generally repeated annually. Less frequent exams (every 2-3 years) may be cost effective after one or more normal eye exams (300-302), while examinations will be required more frequently if retinopathy is progressing.

Examinations can also be done with retinal photographs (with or without dilation of the pupil) read by experienced experts. In-person exams are still necessary when the photos are unacceptable and for follow-up of abnormalities detected. Photos are not a substitute for a comprehensive eye exam, which should be performed at least initially and at intervals thereafter as recommended by an eye care professional. This technology has great potential in areas where qualified eye care professionals are not available and may also enhance efficiency and reduce costs when the expertise of ophthalmologists can be used for more complex examinations and for therapy (303).

Results of eye examinations should be documented and transmitted to the referring health care professional. For a detailed review of the evidence and further discussion of diabetic retinopathy, see the ADA technical review and position statement on this subject $(304,305)$.

\section{Neuropathy screening and treatment (306)}

\section{Recommendations}

- All patients should be screened for distal symmetric polyneuropathy (DPN) at diagnosis and at least annually thereafter using simple clinical tests. (B)

- Electrophysiological testing is rarely needed, except in situations where the clinical features are atypical. (E)

- Screening for signs and symptoms of cardiovascular autonomic neuropathy should be instituted at diagnosis of type 2 diabetes and 5 years after the diagnosis of type 1 diabetes. Special testing is rarely needed and may not affect management or outcomes. (E)

- Medications for the relief of specific symptoms related to DPN and autonomic neuropathy are recommended, as they improve the quality of life of the patient. (E)

The diabetic neuropathies are heterogeneous with diverse clinical manifestations. They may be focal or diffuse. Most common among the neuropathies are chronic sensorimotor DPN and autonomic neuropathy. Although DPN is a diagnosis of exclusion, complex investigations to exclude other conditions are rarely needed.

The early recognition and appropriate management of neuropathy in the patient with diabetes is important for a number of reasons: 1) nondiabetic neuropathies may be present in patients with diabetes and may be treatable; 2 ) a number of treatment options exist for symptomatic diabetic neuropathy; 3) up to $50 \%$ of DPN may be asymptomatic, and patients are at risk of insensate injury to their feet; 4) autonomic neuropathy may involve every system in the body; and 5) cardiovascular autonomic neuropathy causes substantial morbidity and mortality. Specific treatment for the underlying nerve damage is not currently available, other than improved glycemic control, which may slow progression but not reverse neuronal loss. Effective symptomatic treatments are available for some manifestations of DPN and autonomic neuropathy.

\section{Diagnosis of neuropathy}

a. Distal symmetric polyneuropathy. Patients with diabetes should be screened annually for DPN using tests such as pinprick sensation, vibration perception (using a $128-\mathrm{Hz}$ tuning fork), 10-g monofilament pressure sensation at the distal plantar aspect of both great toes and metatarsal joints, and assessment of ankle reflexes. Combinations of more than one test have $>87 \%$ sensitivity in detecting DPN. Loss of 10-g monofilament perception and reduced vibration perception predict foot ulcers (306).

b. Diabetic autonomic neuropathy (307). The symptoms and signs of autonomic dysfunction should be elicited carefully during the history and physical examination. Major clinical manifestations of diabetic autonomic neuropathy include resting tachycardia, exercise intolerance, orthostatic hypotension, constipation, gastroparesis, erectile dysfunction, sudomotor dysfunction, impaired neurovascular function, "brittle diabetes," and hypoglycemic autonomic failure.

Cardiovascular autonomic neuropathy, a CVD risk factor (93), is the most studied and clinically important form of diabetic autonomic neuropathy. Cardiovascular autonomic neuropathy may be indicated by resting tachycardia ( $>100$ bpm), orthostasis (a fall in systolic blood pressure $>20 \mathrm{mmHg}$ upon standing without an appropriate heart rate response), or other disturbances in autonomic nervous system function involving the skin, pupils, or gastrointestinal and genitourinary systems.

Gastrointestinal neuropathies (e.g., esophageal enteropathy, gastroparesis, constipation, diarrhea, and fecal incontinence) are common, and any section of the gastrointestinal tract may be affected. Gastroparesis should be suspected in individuals with erratic glucose control or with upper gastrointestinal symptoms without other identified cause. Evaluation of solid-phase gastric emptying using double-isotope scintigraphy may be done if symptoms are suggestive, but test results often correlate poorly with symptoms. Constipation is the most common lowergastrointestinal symptom but can alternate with episodes of diarrhea.

Diabetic autonomic neuropathy is also associated with genitourinary tract disturbances. In men, diabetic autonomic neuropathy may cause erectile dysfunction and/or retrograde ejaculation. Evaluation of bladder dysfunction should be performed for individuals with diabetes who have recurrent urinary tract infections, pyelonephritis, incontinence, or a palpable bladder. 
Table 16-Table of drugs to treat symptomatic DPN

\begin{tabular}{lll} 
Class & \multicolumn{1}{c}{ Examples } & \multicolumn{1}{c}{ Typical doses* } \\
\hline Tricyclic drugs & Amitriptyline & 10-75 mg at bedtime \\
& Nortriptyline & $25-75 \mathrm{mg}$ at bedtime \\
& Imipramine & $25-75 \mathrm{mg}$ at bedtime \\
Anticonvulsants & Gabapentin & $300-1,200 \mathrm{mg}$ t.i.d. \\
& Carbamazepine & $200-400 \mathrm{mg}$ t.i.d. \\
& Pregabalin ${ }^{\dagger}$ & $100 \mathrm{mg}$ t.i.d. \\
5-Hydroxytryptamine and & Duloxetine $\dagger$ & $60-120 \mathrm{mg}$ daily fs \\
norepinephrine uptake & & \\
inhibitor & & \\
Substance P inhibitor & Capsaicin cream & $0.025-0.075 \%$ applied t.i.d.-q.i.d.
\end{tabular}

*Dose response may vary; initial doses need to be low and titrated up. $†$ Has FDA indication for treatment of painful diabetic neuropathy.

\section{Symptomatic treatments}

a. Distal symmetric polyneuropathy. The first step in management of patients with DPN should be to aim for stable and optimal glycemic control. Although controlled trial evidence is lacking, several observational studies suggest that neuropathic symptoms improve not only with optimization of control, but also with the avoidance of extreme blood glucose fluctuations. Patients with painful DPN may benefit from pharmacological treatment of their symptoms: many agents have efficacy confirmed in published randomized controlled trials, with several FDAapproved for the management of painful DPN. See Table 16 for examples of agents to treat DPN pain.

b. Diabetic autonomic neuropathy. Gastroparesis symptoms may improve with dietary changes and prokinetic agents such as metoclopramide or erythromycin. Treatments for erectile dysfunction may include phosphodiesterase type 5 inhibitors, intracorporeal or intraurethral prostaglandins, vacuum devices, or penile prostheses. Interventions for other manifestations of autonomic neuropathy are described in the ADA statement on neuropathy (306). As with DPN treatments, these interventions do not change the underlying pathology and natural history of the disease process but may have a positive impact on the quality of life of the patient.

\section{E. Foot care}

\section{Recommendations}

- For all patients with diabetes, perform an annual comprehensive foot examination to identify risk factors predictive of ulcers and amputations. The foot examination should include inspection, assessment of foot pulses, and testing for loss of protective sensation (LOPS) (10-g monofilament plus testing any one of: vibration using $128-\mathrm{Hz}$ tuning fork, pinprick sensation, ankle reflexes, or vibration perception threshold). (B)

- Provide general foot self-care education to all patients with diabetes. (B)

- A multidisciplinary approach is recommended for individuals with foot ulcers and high-risk feet, especially those with a history of prior ulcer or amputation. (B) and structural abnormalities, or have history of prior lower-extremity complications to foot care specialists for ongoing preventive care and life-long surveillance. (C)

- Initial screening for peripheral arterial disease (PAD) should include a history for claudication and an assessment of the pedal pulses. Consider obtaining an ankle-brachial index (ABI), as many patients with PAD are asymptomatic. (C)

- Refer patients with significant claudication or a positive ABI for further vascular assessment and consider exercise, medications, and surgical options. (C)

Amputation and foot ulceration, consequences of diabetic neuropathy and/or $\mathrm{PAD}$, are common and major causes of morbidity and disability in people with diabetes. Early recognition and management of risk factors can prevent or delay adverse outcomes.

The risk of ulcers or amputations is increased in people who have the following risk factors:

- previous amputation

- past foot ulcer history

- peripheral neuropathy

- foot deformity

- peripheral vascular disease
- Refer patients who smoke, have LOPS
- visual impairment

- diabetic nephropathy (especially patients on dialysis)

- poor glycemic control

- cigarette smoking

Many studies have been published proposing a range of tests that might usefully identify patients at risk of foot ulceration, creating confusion among practitioners as to which screening tests should be adopted in clinical practice. An ADA task force was therefore assembled in 2008 to concisely summarize recent literature in this area and recommend what should be included in the comprehensive foot exam for adult patients with diabetes. Their recommendations are summarized below, but clinicians should refer to the task force report (308) for further details and practical descriptions of how to perform components of the comprehensive foot examination.

At least annually, all adults with diabetes should undergo a comprehensive foot examination to identify high-risk conditions. Clinicians should ask about history of previous foot ulceration or amputation, neuropathic or peripheral vascular symptoms, impaired vision, tobacco use, and foot care practices. A general inspection of skin integrity and musculoskeletal deformities should be done in a well-lit room. Vascular assessment would include inspection and assessment of pedal pulses.

The neurologic exam recommended is designed to identify LOPS rather than early neuropathy. The clinical examination to identify LOPS is simple and requires no expensive equipment. Five simple clinical tests (use of a 10-g monofilament, vibration testing using a $128-\mathrm{Hz}$ tuning fork, tests of pinprick sensation, ankle reflex assessment, and testing vibration perception threshold with a biothesiometer), each with evidence from wellconducted prospective clinical cohort studies, are considered useful in the diagnosis of LOPS in the diabetic foot. The task force agrees that any of the five tests listed could be used by clinicians to identify LOPS, although ideally two of these should be regularly performed during the screening exam-normally the 10-g monofilament and one other test. One or more abnormal tests would suggest LOPS, while at least two normal tests (and no abnormal test) would rule out LOPS. The last test listed, vibration assessment using a biothesiometer or similar instrument, is widely used in the U.S.; however, 
identification of the patient with LOPS can easily be carried out without this or other expensive equipment.

Initial screening for PAD should include a history for claudication and an assessment of the pedal pulses. A diagnostic ABI should be performed in any patient with symptoms of PAD. Due to the high estimated prevalence of PAD in patients with diabetes and the fact that many patients with PAD are asymptomatic, an ADA consensus statement on PAD (309) suggested that a screening of $\mathrm{ABI}$ be performed in patients over 50 years of age and considered in patients under 50 years of age who have other PAD risk factors (e.g., smoking, hypertension, hyperlipidemia, or duration of diabetes $>10$ years). Refer patients with significant symptoms or a positive $\mathrm{ABI}$ for further vascular assessment and consider exercise, medications, and surgical options (309).

Patients with diabetes and high-risk foot conditions should be educated regarding their risk factors and appropriate management. Patients at risk should understand the implications of the LOPS, the importance of foot monitoring on a daily basis, the proper care of the foot including nail and skin care, and the selection of appropriate footwear. Patients with LOPS should be educated on ways to substitute other sensory modalities (hand palpation, visual inspection) for surveillance of early foot problems. Patients' understanding of these issues and their physical ability to conduct proper foot surveillance and care should be assessed. Patients with visual difficulties, physical constraints preventing movement, or cognitive problems that impair their ability to assess the condition of the foot and to institute appropriate responses will need other people, such as family members, to assist in their care.

People with neuropathy or evidence of increased plantar pressure (e.g., erythema, warmth, callus, or measured pressure) may be adequately managed with well-fitted walking shoes or athletic shoes that cushion the feet and redistribute pressure. Callus can be debrided with a scalpel by a foot care specialist or other health professional with experience and training in foot care. People with bony deformities (e.g., hammertoes, prominent metatarsal heads, or bunions) may need extra-wide or -depth shoes. People with extreme bony deformities (e.g., Charcot foot) who cannot be accom- modated with commercial therapeutic footwear may need custom-molded shoes.

Foot ulcers and wound care may require care by a podiatrist, orthopedic or vascular surgeon, or rehabilitation specialist experienced in the management of individuals with diabetes. For a complete discussion, see the ADA consensus statement on diabetic foot wound care (310).

\section{DIABETES CARE IN SPECIFIC POPULATIONS}

\section{A. Children and adolescents}

\section{Type 1 diabetes}

Three-quarters of all cases of type 1 diabetes are diagnosed in individuals $<18$ years of age. Because children are not simply "small adults," it is appropriate to consider the unique aspects of care and management of children and adolescents with type 1 diabetes. Children with diabetes differ from adults in many respects, including changes in insulin sensitivity related to sexual maturity and physical growth, ability to provide self-care, supervision in child care and school, and unique neurologic vulnerability to hypoglycemia and DKA. Attention to such issues as family dynamics, developmental stages, and physiologic differences related to sexual maturity are all essential in developing and implementing an optimal diabetes regimen. Although recommendations for children and adolescents are less likely to be based on clinical trial evidence, because of current and historical restraints placed on conducting research in children, expert opinion and a review of available and relevant experimental data are summarized in the ADA statement on care of children and adolescents with type 1 diabetes (311).

Ideally, the care of a child or adolescent with type 1 diabetes should be provided by a multidisciplinary team of specialists trained in the care of children with pediatric diabetes. At the very least, education of the child and family should be provided by health care providers trained and experienced in childhood diabetes and sensitive to the challenges posed by diabetes in this age-group. At the time of initial diagnosis, it is essential that diabetes education be provided in a timely fashion, with the expectation that the balance between adult supervision and self-care should be defined by, and will evolve according to, physical, psychological, and emotional maturity. MNT should be provided at diagnosis, and at least annually thereafter, by an individual experienced with the nutritional needs of the growing child and the behavioral issues that have an impact on adolescent diets, including risk for disordered eating.

\section{a. Glycemic control}

\section{Recommendations}

- Consider age when setting glycemic goals in children and adolescents with type 1 diabetes, with less stringent goals for younger children. (E)

While current standards for diabetes management reflect the need to maintain glucose control as near to normal as safely possible, special consideration must be given to the unique risks of hypoglycemia in young children. Glycemic goals need to be modified to take into account the fact that most children $<6$ or 7 years of age have a form of "hypoglycemic unawareness." Their counterregulatory mechanisms are immature and they may lack the cognitive capacity to recognize and respond to hypoglycemic symptoms, placing them at greater risk for severe hypoglycemia and its sequelae. In addition, and unlike the case in adults, young children under the age of 5 years are at risk for permanent cognitive impairment after episodes of severe hypoglycemia (312-314). Extensive evidence indicates that near normalization of blood glucose levels is seldom attainable in children and adolescents after the honeymoon (remission) period. The AlC level achieved in the "intensive" adolescent cohort of the DCCT group was $>1 \%$ higher than that achieved by adult DCCT subjects and above current ADA recommendations for patients in general. However, the increased frequency of use of basal bolus regimens (including insulin pumps) in youth from infancy through adolescence has been associated with more children reaching ADA blood glucose targets $(315,316)$ in those families in which both parents and the child with diabetes are motivated to perform the required diabetes-related tasks.

In selecting glycemic goals, the benefits on long-term health outcomes of achieving a lower AlC must be weighed against the unique risks of hypoglycemia and the difficulties achieving nearnormoglycemia in children and youth. Age-specific glycemic and AlC goals are presented in Table 17. 


\begin{tabular}{|c|c|c|c|c|}
\hline \multirow[b]{2}{*}{ Values by age (years) } & \multicolumn{2}{|c|}{$\begin{array}{l}\text { Plasma blood glucose goal } \\
\text { range }(\mathrm{mg} / \mathrm{dl})\end{array}$} & \multirow[b]{2}{*}{$\mathrm{A} 1 \mathrm{C}$} & \multirow[b]{2}{*}{ Rationale } \\
\hline & Before meals & $\begin{array}{l}\text { Bedtime/ } \\
\text { overnight }\end{array}$ & & \\
\hline Toddlers and preschoolers (0-6) & $100-180$ & $110-200$ & $<8.5 \%$ (but $>7.5 \%)$ & High risk and vulnerability to hypoglycemia \\
\hline School age (6-12) & $90-180$ & $100-180$ & $<8 \%$ & $\begin{array}{l}\text { Risks of hypoglycemia and relatively low risk of } \\
\text { complications prior to puberty }\end{array}$ \\
\hline Adolescents and young adults (13-19) & $90-130$ & $90-150$ & $<7.5 \%$ & $\begin{array}{l}\text { Risk of severe hypoglycemia } \\
\text { Developmental and psychological issues } \\
\text { A lower goal }(<7.0 \%) \text { is reasonable if it can be } \\
\text { achieved without excessive hypoglycemia }\end{array}$ \\
\hline \multicolumn{5}{|c|}{$\begin{array}{l}\text { Key concepts in setting glycemic goals: } \\
\text { - Goals should be individualized and lower goals may be reasonable based on benefit-risk assessment. } \\
\text { - Blood glucose goals should be higher than those listed above in children with frequent hypoglycemia or hypoglycemia unawareness. } \\
\text { - Postprandial blood glucose values should be measured when there is a discrepancy between pre-prandial blood glucose values and AlC } \\
\text { levels and to help assess glycemia in those on basal/bolus regimens. }\end{array}$} \\
\hline
\end{tabular}

\section{b. Screening and management of chronic complications in children and adolescents with type 1 diabetes}

\section{i. Nephropathy}

\section{Recommendations}

- Annual screening for microalbuminuria, with a random spot urine sample for microalbumin-to-creatinine ratio, should be initiated once the child is 10 years of age and has had diabetes for 5 years. (E)

- Confirmed, persistently elevated microalbumin levels on two additional urine specimens should be treated with an ACE inhibitor, titrated to normalization of microalbumin excretion if possible. (E)

\section{ii. Hypertension}

\section{Recommendations}

- Treatment of high-normal blood pressure (systolic or diastolic blood pressure consistently above the 90th percentile for age, sex, and height) should include dietary intervention and exercise aimed at weight control and increased physical activity, if appropriate. If target blood pressure is not reached with 3-6 months of lifestyle intervention, pharmacologic treatment should be initiated. (E)

- Pharmacologic treatment of hypertension (systolic or diastolic blood pressure consistently above the 95th percentile for age, sex, and height or consistently $>130 / 80 \mathrm{mmHg}$, if $95 \%$ exceeds that value) should be initiated as soon as the diagnosis is confirmed. (E)
- ACE inhibitors should be considered for the initial treatment of hypertension. (E)

- The goal of treatment is a blood pressure consistently $<130 / 80$ or below the 90th percentile for age, sex, and height, whichever is lower. (E)

Hypertension in childhood is defined as an average systolic or diastolic blood pressure 95th percentile for age, sex, and height percentile measured on at least three separate days. "High-normal" blood pressure is defined as an average systolic or diastolic blood pressure $\geq 90$ th but $<95$ th percentile for age, sex, and height percentile measured on at least 3 separate days. Normal blood pressure levels for age, sex, and height and appropriate methods for determinations are available online at www.nhlbi.nih.gov/health/ prof/heart/hbp/hbp_ped.pdf.

\section{iii. Dyslipidemia}

\section{Recommendations}

\section{Screening}

- If there is a family history of hypercholesterolemia (total cholesterol >240 $\mathrm{mg} / \mathrm{dl}$ ) or a cardiovascular event before age 55 years, or if family history is unknown, then a fasting lipid profile should be performed on children $>2$ years of age soon after diagnosis (after glucose control has been established). If family history is not of concern, then the first lipid screening should be performed at puberty ( $\geq 10$ years). All children diagnosed with diabetes at or after puberty should have a fasting lipid profile performed soon after diagnosis (after glucose control has been established). (E)

- For both age-groups, if lipids are abnormal, annual monitoring is recommended. If LDL cholesterol values are within the accepted risk levels $(<100$ $\mathrm{mg} / \mathrm{dl}[2.6 \mathrm{mmol} / \mathrm{l}]$ ), a lipid profile should be repeated every 5 years. (E)

\section{Treatment}

- Initial therapy should consist of optimization of glucose control and MNT using a Step II AHA diet aimed at a decrease in the amount of saturated fat in the diet. (E)

- After the age of 10 years, the addition of a statin is recommended in patients who, after MNT and lifestyle changes, have LDL cholesterol $>160 \mathrm{mg} / \mathrm{dl}$ (4.1 $\mathrm{mmol} / \mathrm{l})$ or LDL cholesterol $>130$ $\mathrm{mg} / \mathrm{dl}(3.4 \mathrm{mmol} / \mathrm{l})$ and one or more CVD risk factors. (E)

- The goal of therapy is an LDL cholesterol value $<100 \mathrm{mg} / \mathrm{dl}(2.6 \mathrm{mmol} / \mathrm{l})$. (E)

People diagnosed with type 1 diabetes in childhood have a high risk of early subclinical (317-319) and clinical (320) CVD. Although intervention data are lacking, the AHA categorizes type 1 diabetic children in the highest tier for cardiovascular risk and recommends both lifestyle and pharmacologic treatment for those with elevated LDL cholesterol levels $(321,322)$. Initial therapy should be with a Step II AHA diet, which restricts saturated fat to $7 \%$ of total calories and restricts dietary cholesterol to $200 \mathrm{mg}$ per day. Data from randomized clinical trials 
in children as young as 7 months of age indicate that this diet is safe and does not interfere with normal growth and development $(323,324)$.

For children over the age of 10 years with persistent elevation of LDL cholesterol despite lifestyle therapy, statins should be considered. Neither long-term safety nor cardiovascular outcome efficacy has been established for children. However, recent studies have shown short-term safety equivalent to that seen in adults and efficacy in lowering LDL cholesterol levels, improving endothelial function, and causing regression of carotid intimal thickening (325-327). No statin is approved for use under the age of 10 years, and statin treatment should generally not be used in type 1 diabetic children prior to this age.

\section{iv. Retinopathy}

\section{Recommendations}

- The first ophthalmologic examination should be obtained once the child is 10 years of age and has had diabetes for 3-5 years. (E)

- After the initial examination, annual routine follow-up is generally recommended. Less frequent examinations may be acceptable on the advice of an eye care professional. (E)

Although retinopathy most commonly occurs after the onset of puberty and after 5-10 years of diabetes duration, it has been reported in prepubertal children and with diabetes duration of only 1-2 years. Referrals should be made to eye care professionals with expertise in diabetic retinopathy, an understanding of the risk for retinopathy in the pediatric population, and experience in counseling the pediatric patient and family on the importance of early prevention/intervention.

\section{v. Celiac disease}

\section{Recommendations}

- Children with type 1 diabetes should be screened for celiac disease by measuring tissue transglutaminase or antiendomysial antibodies, with documentation of normal serum IgA levels, soon after the diagnosis of diabetes. (E)

- Testing should be repeated if growth failure, failure to gain weight, weight loss, or gastroenterologic symptoms occur. (E)

- Consideration should be given to peri- odic rescreening of asymptomatic individuals. (E)

- Children with positive antibodies should be referred to a gastroenterologist for evaluation. (E)

- Children with confirmed celiac disease should have consultation with a dietitian and be placed on a gluten-free diet. (E)

Celiac disease is an immune-mediated disorder that occurs with increased frequency in patients with type 1 diabetes (1-16\% of individuals compared with $0.3-1 \%$ in the general population) $(328,329)$. Symptoms of celiac disease include diarrhea, weight loss or poor weight gain, growth failure, abdominal pain, chronic fatigue, malnutrition due to malabsorption, other gastrointestinal problems, and unexplained hypoglycemia or erratic blood glucose concentrations.

\section{vi. Hypothyroidism}

\section{Recommendations}

- Children with type 1 diabetes should be screened for thyroid peroxidase and thyroglobulin antibodies at diagnosis. (E)

- Thyroid-stimulating hormone (TSH) concentrations should be measured after metabolic control has been established. If normal, they should be rechecked every $1-2$ years or if the patient develops symptoms of thyroid dysfunction, thyromegaly, or an abnormal growth rate. Free T4 should be measured if TSH is abnormal. (E)

Autoimmune thyroid disease is the most common autoimmune disorder associated with diabetes, occurring in $17-30 \%$ of patients with type 1 diabetes (330). The presence of thyroid auto-antibodies is predictive of thyroid dysfunction, generally hypothyroidism and less commonly hyperthyroidism (331). Subclinical hypothyroidism may be associated with increased risk of symptomatic hypoglycemia (332) and with reduced linear growth (333). Hyperthyroidism alters glucose metabolism, potentially resulting in deterioration of metabolic control.

c. Self-management. No matter how sound the medical regimen, it can only be as good as the ability of the family and/or individual to implement it. Family involvement in diabetes remains an important component of optimal diabetes management throughout childhood and into adolescence. Health care providers who care for children and adolescents therefore must be capable of evaluating the behavioral, emotional, and psychosocial factors that interfere with implementation and then must work with the individual and family to resolve problems that occur and/or to modify goals as appropriate.

d. School and day care. Since a sizable portion of a child's day is spent in school, close communication with school or day care personnel is essential for optimal diabetes management, safety, and maximal academic opportunities. See VIII.B. Diabetes Care in the School and Day Care Setting, for further discussion.

\section{Type 2 diabetes}

The incidence of type 2 diabetes in adolescents is increasing, especially in ethnic minority populations (21). Distinction between type 1 and type 2 diabetes in children can be difficult, since the prevalence of overweight in children continues to rise and since autoantigens and ketosis may be present in a substantial number of patients with features of type 2 diabetes (including obesity and acanthosis nigricans). Such a distinction at the time of diagnosis is critical because treatment regimens, educational approaches, and dietary counsel will differ markedly between the two diagnoses.

Type 2 diabetes has a significant incidence of comorbidities already present at the time of diagnosis (334). It is recommended that blood pressure measurement, a fasting lipid profile, microalbuminuria assessment, and dilated eye examination be performed at the time of diagnosis. Thereafter, screening guidelines and treatment recommendations for hypertension, dyslipidemia, microalbuminuria, and retinopathy in youth with type 2 diabetes are similar to those for youth with type 1 diabetes. Additional problems that may need to be addressed include polycystic ovary disease and the various comorbidities associated with pediatric obesity such as sleep apnea, hepatic steatosis, orthopedic complications, and psychosocial concerns. The ADA consensus statement on this subject (23) provides guidance on the prevention, screening, and treatment of type 2 diabetes and its comorbidities in young people. 


\section{B. Preconception care}

\section{Recommendations}

- AlC levels should be as close to normal as possible $(<7 \%)$ in an individual patient before conception is attempted. (B)

- Starting at puberty, preconception counseling should be incorporated in the routine diabetes clinic visit for all women of child-bearing potential. (C)

- Women with diabetes who are contemplating pregnancy should be evaluated and, if indicated, treated for diabetic retinopathy, nephropathy, neuropathy, and CVD. (E)

- Medications used by such women should be evaluated prior to conception because drugs commonly used to treat diabetes and its complications may be contraindicated or not recommended in pregnancy, including statins, ACE inhibitors, ARBs, and most noninsulin therapies. (E)

Major congenital malformations remain the leading cause of mortality and serious morbidity in infants of mothers with type 1 or type 2 diabetes. Observational studies indicate that the risk of malformations increases continuously with increasing maternal glycemia during the first 6-8 weeks of gestation, as defined by firsttrimester A1C concentrations. There is no threshold for AlC values below which risk disappears entirely. However, malformation rates above the 1-2\% background rate of nondiabetic pregnancies appear to be limited to pregnancies in which first-trimester AlC concentrations are $>1 \%$ above the normal range for a nondiabetic pregnant woman.

Preconception care of diabetes appears to reduce the risk of congenital malformations. Five nonrandomized studies compared rates of major malformations in infants between women who participated in preconception diabetes care programs and women who initiated intensive diabetes management after they were already pregnant. The preconception care programs were multidisciplinary and designed to train patients in diabetes selfmanagement with diet, intensified insulin therapy, and SMBG. Goals were set to achieve normal blood glucose concentrations, and $>80 \%$ of subjects achieved normal AlC concentrations before they became pregnant (335-339). In all five studies, the incidence of major congenital malformations in women who participated in preconception care (range 1.0
$1.7 \%$ of infants) was much lower than the incidence in women who did not participate (range 1.4-10.9\% of infants). One limitation of these studies is that participation in preconception care was selfselected rather than randomized. Thus, it is impossible to be certain that the lower malformation rates resulted fully from improved diabetes care. Nonetheless, the evidence supports the concept that malformations can be reduced or prevented by careful management of diabetes before pregnancy.

Planned pregnancies greatly facilitate preconception diabetes care. Unfortunately, nearly two-thirds of pregnancies in women with diabetes are unplanned, leading to a persistent excess of malformations in infants of diabetic mothers. To minimize the occurrence of these devastating malformations, standard care for all women with diabetes who have childbearing potential, beginning at the onset of puberty or at diagnosis, should include 1) education about the risk of malformations associated with unplanned pregnancies and poor metabolic control; and 2) use of effective contraception at all times, unless the patient has good metabolic control and is actively trying to conceive.

Women contemplating pregnancy need to be seen frequently by a multidisciplinary team experienced in the management of diabetes before and during pregnancy. The goals of preconception care are to 1) involve and empower the patient in the management of her diabetes, 2) achieve the lowest AlC test results possible without excessive hypoglycemia, 3) assure effective contraception until stable and acceptable glycemia is achieved, and 4) identify, evaluate, and treat longterm diabetes complications such as retinopathy, nephropathy, neuropathy, hypertension, and CHD (76).

Among the drugs commonly used in the treatment of patients with diabetes, a number may be relatively or absolutely contraindicated during pregnancy. Statins are category $\mathrm{X}$ (contraindicated for use in pregnancy) and should be discontinued before conception, as should ACE inhibitors (340). ARBs are category C (risk cannot be ruled out) in the first trimester but category D (positive evidence of risk) in later pregnancy and should generally be discontinued before pregnancy. Among the oral antidiabetic agents, metformin and acarbose are classified as category B (no evidence of risk in humans) and all others as category C. Potential risks and benefits of oral antidia- betic agents in the preconception period must be carefully weighed, recognizing that data are insufficient to establish the safety of these agents in pregnancy.

For further discussion of preconception care, see the related ADA consensus statement (76) and position statement (341) on preexisting diabetes and pregnancy.

\section{Older adults}

\section{Recommendations}

- Older adults who are functional, are cognitively intact, and have significant life expectancy should receive diabetes care using goals developed for younger adults. (E)

- Glycemic goals for older adults not meeting the above criteria may be relaxed using individual criteria, but hyperglycemia leading to symptoms or risk of acute hyperglycemic complications should be avoided in all patients. (E)

- Other cardiovascular risk factors should be treated in older adults with consideration of the time frame of benefit and the individual patient. Treatment of hypertension is indicated in virtually all older adults, and lipid and aspirin therapy may benefit those with life expectancy at least equal to the time frame of primary or secondary prevention trials. (E)

- Screening for diabetes complications should be individualized in older adults, but particular attention should be paid to complications that would lead to functional impairment. (E)

Diabetes is an important health condition for the aging population; at least 20\% of patients over the age of 65 years have diabetes, and this number can be expected to grow rapidly in the coming decades. Older individuals with diabetes have higher rates of premature death, functional disability, and coexisting illnesses such as hypertension, CHD, and stroke than those without diabetes. Older adults with diabetes are also at greater risk than other older adults for several common geriatric syndromes, such as polypharmacy, depression, cognitive impairment, urinary incontinence, injurious falls, and persistent pain.

The American Geriatric Society's guidelines for improving the care of the older person with diabetes (342) have influenced the following discussion and recommendations. The care of older 
adults with diabetes is complicated by their clinical and functional heterogeneity. Some older individuals developed diabetes years earlier and may have significant complications; others who are newly diagnosed may have had years of undiagnosed diabetes with resultant complications or may have few complications from the disease. Some older adults with diabetes are frail and have other underlying chronic conditions, substantial diabetes-related comorbidity, or limited physical or cognitive functioning. Other older individuals with diabetes have little comorbidity and are active. Life expectancies are highly variable for this population but often longer than clinicians realize. Providers caring for older adults with diabetes must take this heterogeneity into consideration when setting and prioritizing treatment goals

There are few long-term studies in older adults that demonstrate the benefits of intensive glycemic, blood pressure, and lipid control. Patients who can be expected to live long enough to reap the benefits of long-term intensive diabetes management and who are active, have good cognitive function, and are willing should be provided with the needed education and skills to do so and be treated using the goals for younger adults with diabetes.

For patients with advanced diabetes complications, life-limiting comorbid illness, or substantial cognitive or functional impairment, it is reasonable to set less-intensive glycemic target goals. These patients are less likely to benefit from reducing the risk of microvascular complications and more likely to suffer serious adverse effects from hypoglycemia. However, patients with poorly controlled diabetes may be subject to acute complications of diabetes, including dehydration, poor wound healing, and hyperglycemic hyperosmolar coma. Glycemic goals at a minimum should avoid these consequences.

Although control of hyperglycemia may be important in older individuals with diabetes, greater reductions in morbidity and mortality may result from control of other cardiovascular risk factors rather than from tight glycemic control alone. There is strong evidence from clinical trials of the value of treating hypertension in the elderly $(343,344)$. There is less evidence for lipid-lowering and aspirin therapy, although the benefits of these interventions for primary and secondary prevention are likely to apply to older adults whose life expectancies equal or exceed the time frames seen in clinical trials.

Special care is required in prescribing and monitoring pharmacologic therapy in older adults. Metformin is often contraindicated because of renal insufficiency or significant heart failure. TZDs can cause fluid retention, which may exacerbate or lead to heart failure. They are contraindicated in patients with CHF (New York Heart Association class III and IV), and if used at all should be used very cautiously in those with, or at risk for, milder degrees of CHF. Sulfonylureas, other insulin secretagogues, and insulin can cause hypoglycemia. Insulin use requires that patients or caregivers have good visual and motor skills and cognitive ability. Drugs should be started at the lowest dose and titrated up gradually until targets are reached or side effects develop.

Screening for diabetes complications in older adults also should be individualized. Particular attention should be paid to complications that can develop over short periods of time and/or that would significantly impair functional status, such as vision and lower-extremity complications.

\section{Cystic fibrosis-related diabetes}

Cystic fibrosis-related diabetes (CFRD) is the most common comorbidity in people with cystic fibrosis, occurring in 20\% of adolescents and $40-50 \%$ of adults. The additional diagnosis of diabetes in this population is associated with worse nutritional status, more severe inflammatory lung disease, and greater mortality from respiratory failure. For reasons that are not well understood, women with CFRD are particularly vulnerable to excess morbidity and mortality. Insulin insufficiency related to partial fibrotic destruction of the islet mass is the primary defect in CFRD. Genetically determined function of the remaining $\beta$-cells and insulin resistance associated with infection and inflammation may also play a role. Encouraging new data suggest that early detection and aggressive insulin therapy have narrowed the gap in mortality between cystic fibrosis patients with and without diabetes and have eliminated the sex difference in mortality.

A consensus conference on CFRD was cosponsored in 2009 by ADA, the Cystic Fibrosis Foundation, and the Lawson Wilkins Pediatric Endocrine Society. Recommendations for the clinical man- agement of CFRD will be found in the consensus report to be published in 2010.

\section{DIABETES CARE IN SPECIFIC SETTINGS}

\section{Diabetes care in the hospital}

\section{Recommendations}

- All patients with diabetes admitted to the hospital should have their diabetes clearly identified in the medical record. (E)

- All patients with diabetes should have an order for blood glucose monitoring, with results available to all members of the health care team. (E)

- Goals for blood glucose levels

- Critically ill patients: Insulin therapy should be initiated for treatment of persistent hyperglycemia starting at a threshold of $\leq 180 \mathrm{mg} / \mathrm{dl}$ (10 mmol/ 1). Once insulin therapy is started, a glucose range of $140-180 \mathrm{mg} / \mathrm{dl}$ $(7.8-10 \mathrm{mmol} / \mathrm{l})$ is recommended for the majority of critically ill patients. (A) These patients require an intravenous insulin protocol that has demonstrated efficacy and safety in achieving the desired glucose range without increasing risk for severe hypoglycemia. (E)

- Non-critically ill patients: There is no clear evidence for specific blood glucose goals. If treated with insulin, the premeal blood glucose target should generally be $<140 \mathrm{mg} / \mathrm{dl}$ (7.8 $\mathrm{mmol} / \mathrm{l}$ ) with random blood glucose $<180 \mathrm{mg} / \mathrm{dl}$ (10.0 mmol/l), provided these targets can be safely achieved. More stringent targets may be appropriate in stable patients with previous tight glycemic control. Less stringent targets may be appropriate in those with severe comorbidites. (E)

- Scheduled subcutaneous insulin with basal, nutritional, and correction components is the preferred method for achieving and maintaining glucose control in noncritically ill patients. (C) Using correction dose or "supplemental" insulin to correct premeal hyperglycemia in addition to scheduled prandial and basal insulin is recommended. (E)

- Glucose monitoring should be initiated in any patient not known to be diabetic who receives therapy associated with high risk for hyperglycemia, including high-dose glucocorticoid therapy, initiation of enteral or parenteral nutrition, or other medications such as octreotide 
or immunosuppressive medications (B) If hyperglycemia is documented and persistent, treatment is necessary. Such patients should be treated to the same glycemic goals as patients with known diabetes. (E)

- A plan for treating hypoglycemia should be established for each patient. Episodes of hypoglycemia in the hospital should be tracked. (E)

- All patients with diabetes admitted to the hospital should have an AlC obtained if the result of testing in the previous 2-3 months is not available. (E)

- Patients with hyperglycemia in the hospital who do not have a diagnosis of diabetes should have appropriate plans for follow-up testing and care documented at discharge. (E)

The subject of diabetes in the hospital is extensively reviewed in an ADA technical review (345). A recent updated consensus statement by the American Association of Clinical Endocrinologists (AACE) and the ADA (346) form the basis for the discussion and guidelines in this section.

The literature on hospitalized patients with hyperglycemia typically describes three categories:

- Medical history of diabetes: diabetes previously diagnosed and acknowledged by the patient's treating physician.

- Unrecognized diabetes: hyperglycemia (fasting blood glucose $\geq 126 \mathrm{mg} / \mathrm{dl}$ or random blood glucose $\geq 200 \mathrm{mg} / \mathrm{dl}$ ) occurring during hospitalization and confirmed as diabetes after hospitalization by standard diagnostic criteria but unrecognized as diabetes by the treating physician during hospitalization.

- Hospital-related hyperglycemia: hyperglycemia (fasting blood glucose $\geq 126$ $\mathrm{mg} / \mathrm{dl}$ or random blood glucose $\geq 200$ $\mathrm{mg} / \mathrm{dl}$ ) occurring during the hospitalization that reverts to normal after hospital discharge.

The management of hyperglycemia in the hospital has logically been considered secondary in importance to the condition that prompted admission (345). However, a body of literature now supports targeted glucose control in the hospital setting for potential improved clinical outcomes. Hyperglycemia in the hospital may result from stress; decompensation of type 1 , type 2 , or other forms of diabetes; and/or may be iatrogenic due to withholding of antihyperglycemic medications or administration of hyper- glycemia-provoking agents such as glucocorticoids or vasopressors.

People with diabetes are more likely to be hospitalized and to have longer lengths of stay than those without diabetes. A recent survey estimated that $22 \%$ of all hospital inpatient days were incurred by people with diabetes and that hospital inpatient care accounted for one-half of the $\$ 174$ billion total U.S. medical expenditures for this disease (347). This is due, in part, to the continued expansion of the worldwide epidemic of type 2 diabetes. In the U.S. alone, there are $\sim 1.6$ million new cases of diabetes each year with an overall prevalence of 23.6 million people $(7.8 \%$ of the population, with one-quarter of cases remaining undiagnosed). An additional 57 million American adults are at high risk for type 2 diabetes (348). While the costs of illness-related stress hyperglycemia are not known, they are likely to be significant given the poor prognosis of such patients (349-352).

There is substantial observational evidence linking hyperglycemia in hospitalized patients (with or without diabetes) to poor outcomes. Cohort studies as well as a few early randomized controlled trials (RCTs) suggested that intensive treatment of hyperglycemia improved hospital outcomes $(345,351,352)$. Interventions to normalize glycemia, however, have had inconsistent results. Indeed, recent trials in critically ill patients have failed to show a significant improvement in mortality with intensive glycemic control $(353,354)$ or have even shown increased mortality risk (355). Moreover, these recent RCTs have highlighted the risk of severe hypoglycemia resulting from such efforts (353-358).

The largest study to date, NICESUGAR, a multicenter, multinational RCT, tested the effect of tight glycemic control (target $81-108 \mathrm{mg} / \mathrm{dl}$ ) on outcomes among 6,104 critically ill participants, the majority of whom (>95\%) required mechanical ventilation (355). Ninety-day mortality was significantly higher in the intensive versus the conventional group (target $144-180 \mathrm{mg} / \mathrm{dl}$ ) (78 more deaths; 27.5 vs. $24.9 \%, P=0.02$ ) in both surgical and medical patients. Mortality from cardiovascular causes was more common in the intensive group (76 more deaths; 41.6 vs. $35.8 \% ; P=0.02$ ). Severe hypoglycemia was also more common in the intensively treated group (6.8 vs. $0.5 \% ; P<0.001)$. The precise reason for the increased mortality in the tightly controlled group is unknown. The results of this study lie in stark contrast to a famous 2001 single-center study that reported a $42 \%$ relative reduction in intensive care unit (ICU) mortality in critically ill surgical patients treated to a target blood glucose of $80-110 \mathrm{mg} / \mathrm{dl}$. Importantly, the control group in NICESUGAR had reasonably good blood glucose management, maintained at a mean glucose of $144 \mathrm{mg} / \mathrm{dl}$, only $29 \mathrm{mg} / \mathrm{dl}$ above the intensively managed patients. Accordingly, this study's findings do not disprove the notion that glycemic control in the ICU is important. However, they do strongly suggest that it is not necessary to target blood glucose values $<140 \mathrm{mg} / \mathrm{dl}$ and that a highly stringent target of $<110$ $\mathrm{mg} / \mathrm{dl}$ actually may be dangerous.

In a recent meta-analysis of 26 trials $(N=13,567)$, which included the NICESUGAR data, the pooled relative risk (RR) of death with intensive insulin therapy was 0.93 as compared with conventional therapy (95\% CI 0.83-1.04) (358). Approximately half of these trials reported hypoglycemia, with a pooled RR of intensive therapy of 6.0 (95\% CI 4.5-8.0). The specific ICU setting influenced the findings, with patients in surgical ICUs appearing to benefit from intensive insulin therapy (RR 0.63 [95\% CI 0.44-0.91]), while those in other critical care settings did not (medical ICU: 1.0 [0.78-1.28]; "mixed" ICU: 0.99 [0.86-1.12]). It was concluded that overall, intensive insulin therapy increased the risk of hypoglycemia but provided no overall benefit on mortality in the critically ill, although a benefit to patients admitted to the surgical ICU was suggested.

It is very clear that the management of hyperglycemia in the hospital presents unique challenges that stem from variations in a patient's nutritional status and level of consciousness, the practical limitations of intermittent glycemic monitoring, and the ultimate importance of patient safety. Accordingly, reasonable glucose targets in the hospital setting are modestly higher than may be routinely advised in patients with diabetes in the outpatient setting. The following recommendations represent a synthesis of the evidence base over the past decade and are somewhat less stringent than prior recommendations of the ADA Standards of Medical Care in Diabetes. For a comprehensive review of these data, the reader is referred to the latest consensus statement from AACE and ADA on inpatient management of hyperglycemia (346) 
1. Glycemic targets in hospitalized patients

a. Definition of glucose abnormalities in the hospital setting. Hyperglycemia has been defined as any blood glucose $>140$ $\mathrm{mg} / \mathrm{dl}(7.8 \mathrm{mmol} / \mathrm{l})$. Levels that are significantly and persistently above this may require treatment in hospitalized patients. In patients without a previous diagnosis of diabetes, elevated blood glucose may be due to "stress hyperglycemia," a condition that can be established by a review of prior records or measurement of an AlC. $\mathrm{AlC}$ values $>6.5 \%$ suggest that diabetes preceded hospitalization (359). Hypoglycemia has been defined as any blood glucose $<70 \mathrm{mg} / \mathrm{dl}$ ( $3.9 \mathrm{mmol} / \mathrm{l})$. This is the standard definition in outpatients and correlates with the initial threshold for the release of counterregulatory hormones (177). Severe hypoglycemia in hospitalized patients has been defined by many as $<40 \mathrm{mg} / \mathrm{dl}(2.2 \mathrm{mmol} / \mathrm{l})$, although this is lower than the $\sim 50 \mathrm{mg} / \mathrm{dl}(2.8$ $\mathrm{mmol} / \mathrm{l}$ ) level at which cognitive impairment begins in normal individuals $(177,360,361)$. As with hyperglycemia, hypoglycemia among inpatients is also associated with adverse short- and longterm outcomes. Early recognition and treatment of mild to moderate hypoglycemia (40 and $69 \mathrm{mg} / \mathrm{dl} \mathrm{[2.2} \mathrm{and} 3.8 \mathrm{mmol} /$ 1]) can prevent deterioration to a more severe episode with potential adverse sequelae $(361,362)$.

$i$. Critically ill patients. Based on the weight of the available evidence, for the majority of critically ill patients in the ICU setting, insulin infusion should be used to control hyperglycemia, with a starting threshold of $\leq 180 \mathrm{mg} / \mathrm{dl}(10.0 \mathrm{mmol} / \mathrm{l})$. Once intravenous insulin is started, the glucose level should be maintained between 140 and $180 \mathrm{mg} / \mathrm{dl}(7.8$ and 10.0 $\mathrm{mmol} / \mathrm{l})$. Greater benefit may be realized at the lower end of this range. Although strong evidence is lacking, somewhat lower glucose targets may be appropriate in selected patients. However, targets $<110 \mathrm{mg} / \mathrm{dl}(6.1 \mathrm{mmol} / \mathrm{l})$ are not recommended. Use of insulin infusion protocols with demonstrated safety and efficacy, resulting in low rates of hypoglycemia, are highly recommended.

ii. Noncritically ill patients. With no prospective, RCT data to inform specific glycemic targets in noncritically ill patients, recommendations are based on clinical experience and judgment. For the majority of noncritically ill patients treated with insulin, premeal glucose targets should generally be $<140 \mathrm{mg} / \mathrm{dl}(7.8 \mathrm{mmol} / \mathrm{l})$ with random blood glucose $<180 \mathrm{mg} / \mathrm{dl}$ (10.0 mmol/1), as long as these targets can be safely achieved. To avoid hypoglycemia, consideration should be given to reassessing the insulin regimen if blood glucose levels fall below $100 \mathrm{mg} / \mathrm{dl}(5.6$ $\mathrm{mmol} / \mathrm{l})$. Modification of the regimen is required when blood glucose values are $<70 \mathrm{mg} / \mathrm{dl}$ (3.9 mmol/l), unless the event is easily explained by other factors (such as a missed meal, etc.).

Occasional patients with a prior history of successful tight glycemic control in the outpatient setting who are clinically stable may be maintained with a glucose range below the above cut points. Conversely, higher glucose ranges may be acceptable in terminally ill patients or in patients with severe comorbidities, as well as in those in patient-care settings where frequent glucose monitoring or close nursing supervision is not feasible.

Clinical judgment, combined with ongoing assessment of the patient's clinical status, including changes in the trajectory of glucose measures, severity of illness, nutritional status, or concurrent use of medications that might affect glucose levels (e.g., steroids, octreotide) must be incorporated into the day-to-day decisions regarding insulin dosing (363).

\section{Treatment options in hospitalized patients}

In the hospital setting, insulin therapy is the preferred method of glycemic control in majority of clinical situations (346). In the ICU, intravenous infusion is the preferred route of insulin administration. Outside of critical care units, subcutaneous insulin is used much more frequently. Oral agents have a limited role in the inpatient setting.

a. Intravenous insulin infusions. In the critical care setting, continuous intravenous insulin infusion has been shown to be the most effective method for achieving specific glycemic targets (346). Because of the very short half-life of circulating insulin, intravenous delivery allows rapid dosing adjustments to address alterations in patients' status.

Intravenous insulin is ideally administered via validated written or computerized protocols that allow for predefined adjustments to the insulin infusion rate according to glycemic fluctuations and insulin dose. An extensive review of the merits and deficiencies of published protocols is beyond the intent of this statement, and the reader is referred to several available reports and reviews (364-366).
Continued education of staff with periodic ongoing review of patient data are critical for successful implementation of any insulin protocol (364-366).

Patients who receive intravenous insulin infusion will usually require transition to subcutaneous insulin when they begin eating regular meals or are transferred to lower intensity care. Typically, a percentage (usually $75-80 \%$ ) of the total daily intravenous infusion dose is proportionately divided into basal and prandial components (see below). Importantly, subcutaneous insulin must be given 1-4 $\mathrm{h}$ prior to discontinuation of intravenous insulin to prevent hyperglycemia (367).

b. Subcutaneous insulin. Scheduled subcutaneous insulin is the preferred method for achieving and maintaining glucose control in non-ICU patients with diabetes or stress hyperglycemia. The recommended components of inpatient subcutaneous insulin regimens include a basal, nutritional, and supplemental (correction) component $(345,346,368)$. Each component can be met by one of several available insulin products, depending on the particular hospital situation. The reader is referred to several recent publications and reviews that describe currently available insulin preparations and protocols (366-370).

A topic that deserves particular attention is the persistent overuse of what has been branded as sliding scale insulin (SSI) for management of hyperglycemia. The term "correction insulin," which refers to the use of additional short or rapid-acting insulin with scheduled insulin doses to treat blood glucose above desired targets, is preferred (345). Prolonged therapy with SSI as the sole regimen is ineffective in the majority of patients (and potentially dangerous in type 1 diabetes) (370-375). c. Noninsulin agents. These agents are inappropriate in the majority of hospitalized patients because they are less titratable than insulin in the short tem and are meant to be used in patients eating on a regular meal schedule. Continuation of these agents may be appropriate in selected stable patients who are expected to consume meals at regular intervals. Specific caution is required with metformin, due to the possibility that a contraindication may develop during the hospitalization, such as renal insufficiency, unstable hemodynamic status, or need for an imaging study that requires a radio-contrast dye $(345,376)$. Injectable noninsulin therapies such as exenatide and pramlint- 
ide have limitations similar to those of oral agents in the hospital setting.

\section{d. Specific clinical situations}

i. Insulin pumps. Patients who use CSII pump therapy in the outpatient setting can be candidates for diabetes selfmanagement in the hospital, provided that they have the mental and physical capacity to do so $(346,368)$. It is important that nursing personnel document basal rates and bolus doses on a regular basis (at least daily). The availability of hospital personnel with expertise in CSII therapy is essential.

ii. Enteral nutrition. Hyperglycemia is a common side effect of inpatient enteral nutrition therapy (377). A recent report using a combination of basal insulin with correction insulin achieved a mean glucose value of $160 \mathrm{mg} / \mathrm{dl}$ (8.9 mmol/l). Similar results were achieved in the group randomized to receive SSI alone; however, $48 \%$ of patients required the addition of intermediate-acting insulin to achieve glycemic targets (373).

iii. Parenteral nutrition. The high glucose load in standard parenteral nutrition frequently results in hyperglycemia, which is associated with a higher incidence of complications and mortality in critically ill ICU patients (378). Insulin therapy is highly recommended, with glucose targets as defined previously by severity of illness.

iv. Glucocorticoid therapy. Hyperglycemia is a common complication of corticosteroid therapy (363). Several approaches have been proposed for treatment of this condition, but there are no published protocols or studies that investigate the efficacy of these approaches. A reasonable approach is to institute glucose monitoring for at least $48 \mathrm{~h}$ in all patients receiving high dose glucocorticoid therapy and initiate insulin as appropriate. In patients who are already being treated for hyperglycemia, early adjustment of insulin doses is recommended. Importantly, during steroid tapers, insulin dosing should be proactively adjusted to avoid hypoglycemia.

v. Hypoglycemia prevention. Hypoglycemia, especially in insulin-treated patients, is the leading limiting factor in the glycemic management of type 1 and type 2 diabetes (174). In the hospital, multiple additional risk factors for hypoglycemia are present, even among patients who are neither "brittle" nor tightly controlled. Patients with or without diabetes may experience hypoglycemia in the hospital in association with altered nutritional state, heart failure, renal or liver disease, malignancy, infection, or sepsis $(379,379,380)$. Additional triggering events leading to iatrogenic hypoglycemia include sudden reduction of corticosteroid dose, altered ability of the patient to self-report symptoms, reduction of oral intake, emesis, new NPO status, inappropriate timing of short- or rapid-acting insulin in relation to meals, reduction of rate of administration of intravenous dextrose, and unexpected interruption of enteral feedings or parenteral nutrition.

Despite the preventable nature of many inpatient episodes of hypoglycemia, institutions are more likely to have nursing protocols for the treatment of hypoglycemia than for its prevention. Tracking such episodes and analyzing their causes are important quality improvement activities.

\section{Diabetes care providers in the hospital}

Inpatient diabetes management may be effectively provided by primary care physicians, endocrinologists, or hospitalists. Involvement of appropriately trained specialists or specialty teams may reduce length of stay, improve glycemic control, and improve outcomes (381-384). In the care of diabetes, implementation of standardized order sets for scheduled and correction-dose insulin may reduce reliance on sliding-scale management. A team approach is needed to establish hospital pathways. To achieve glycemic targets associated with improved hospital outcomes, hospitals will need multidisciplinary support to develop protocols for subcutaneous insulin therapy that effectively and safely achieve glycemic targets (385).

\section{Self-management in the hospital}

Self-management of diabetes in the hospital may be appropriate for competent adult patients who have a stable level of consciousness, have reasonably stable daily insulin requirements, successfully conduct self-management of diabetes at home, have physical skills needed to successfully self-administer insulin and perform SMBG, have adequate oral intake, and are proficient in carbohydrate counting, use of multiple daily insulin injections, or insulin pump therapy and sickday management. The patient and physician, in consultation with nursing staff, must agree that patient selfmanagement is appropriate under the conditions of hospitalization. For patients conducting self-management in the hospital, it is imperative that basal, prandial, and correction doses of insulin and results of bedside glucose monitoring be recorded as part of the patient's hospital medical record. While many institutions allow patients on insulin pumps to continue these devices in the hospital, others express concern regarding use of a device unfamiliar to staff, particularly in patients who are not able to manage their own pump therapy. If a patient is too ill to self-manage either multiple daily injections or CSII, then appropriate subcutaneous doses can be calculated on the basis of their basal and bolus insulin needs during hospitalization, with adjustments for changes in nutritional or metabolic status.

\section{DSME in the hospital}

Teaching diabetes self-management to patients in hospitals is a challenging task. Patients are ill, under increased stress related to their hospitalization and diagnosis, and in an environment not conducive to learning. Ideally, people with diabetes should be taught at a time and place conducive to learning - as an outpatient in a recognized program of diabetes education.

For the hospitalized patient, diabetes "survival skills" education is generally a feasible approach. Patients and/or family members receive sufficient information and training to enable safe care at home. Those newly diagnosed with diabetes or who are new to insulin and/or blood glucose monitoring need to be instructed before discharge. Those patients hospitalized because of a crisis related to diabetes management or poor care at home need education to prevent subsequent episodes of hospitalization. An assessment of the need for a home health referral or referral to an outpatient diabetes education program should be part of discharge planning for all patients.

\section{MNT in the hospital}

Hospital diets continue to be ordered by calorie levels based on the "ADA diet." However, since 1994 the ADA has not endorsed any single meal plan or specified percentages of macronutrients, and the term "ADA diet" should no longer be used. Current nutrition recommendations advise individualization based on treatment goals, physiologic parameters, and medication usage. Because of the complexity of nutrition issues in the hospital, a registered dietitian, knowledgeable and skilled in MNT, should serve as 
an inpatient team member. The dietitian is responsible for integrating information about the patient's clinical condition, eating, and lifestyle habits and for establishing treatment goals in order to determine a realistic plan for nutrition therapy $(386,387)$.

\section{Bedside blood glucose monitoring}

Bedside blood glucose monitoring using point-of-care glucose meters is performed before meals and bedtime in the majority of inpatients who are eating usual meals. In patients who are receiving continuous enteral or parenteral nutrition, glucose monitoring is optimally performed every 4-6 h. In patients who are receiving cycled enteral or parenteral nutrition, the schedule for glucose monitoring can be individualized but should be frequent enough to detect hyperglycemia during feedings and risk of hypoglycemia when feedings are interrupted $(374,376)$. More frequent blood glucose testing ranging from every $30 \mathrm{~min}$ to every $2 \mathrm{~h}$ is required for patients on intravenous insulin infusions.

Safe and rational glycemic management relies on the accuracy of blood glucose measurements using point-of-care blood glucose meters, which have several important limitations. Although the FDA allows a $\pm 20 \%$ error for glucose meters, questions about the appropriateness of this criterion have been raised (388). Glucose measures differ significantly between plasma and whole blood, terms which are often used interchangeably and can lead to misinterpretation. Most commercially available capillary glucose meters introduce a correction factor of $\sim 1.12$ to report a "plasma-adjusted" value (389).

Significant discrepancies between capillary, venous, and arterial plasma samples have been observed in patients with low or high hemoglobin concentrations, hypoperfusion, and the presence of interfering substances $(389,390)$. Analytical variability has been described with several point-of-care meters (391). Any glucose result that does not correlate with the patient's status should be confirmed through conventional laboratory sampling of $P G$.

While laboratory measurement of PG has less variability and interference, multiple daily phlebotomies are not practical. The use of indwelling lines as the sampling source also poses risks for infection. Studies performed using continuous interstitial glucose monitoring systems in the critical care setting (392) currently are limited by the lack of reliability in the hypoglycemic range as well as by cost.

\section{Discharge planning}

It is important to anticipate the postdischarge antihyperglycemic regimen in all patients with diabetes or newly discovered hyperglycemia. The optimal program will need to consider the type and severity of diabetes, the effects of the patient's illness on blood glucose levels, and the capacities and desires of the patient. Smooth transition to outpatient care should be ensured, especially in those new to insulin therapy or in whom the diabetes regimen has been substantially altered during the hospitalization. All patients in whom the diagnosis of diabetes is new should have, at minimum, "survival skills" training prior to discharge.

It is recommended that the following areas be reviewed and addressed prior to hospital discharge:

- level of understanding related to the diagnosis of diabetes

- SMBG and explanation of home blood glucose goals

- definition, recognition, treatment, and prevention of hyperglycemia and hypoglycemia

- identification of health care provider who will provide diabetes care after discharge

- information on consistent eating patterns

- when and how to take blood glucoselowering medications including insulin administration (if going home on insulin)

- sick-day management

- proper use and disposal of needles and syringes

More expanded diabetes education can be arranged in the community. An outpatient follow-up visit with the primary care provider, endocrinologist, or diabetes educator within 1 month of discharge is advised for all patients having hyperglycemia in the hospital. Clear communication with outpatient providers either directly or via hospital discharge summaries facilitates safe transitions to outpatient care. Providing information regarding the cause or the plan for determining the cause of hyperglycemia, related complications and comorbidities, and recommended treatments can assist outpatient providers as they assume ongoing care.

\section{STRATECIES FOR IMPROVING DIABETES}

CARE - The implementation of the standards of care for diabetes has been suboptimal in most clinical settings. A recent report (393) indicated that only $57.1 \%$ of adults with diagnosed diabetes achieved an AlC of $<7 \%$, only $45.5 \%$ had a blood pressure $<130 / 80 \mathrm{mmHg}$, and just $46.5 \%$ had a total cholesterol $<200 \mathrm{mg} / \mathrm{dl}$. Most distressing was that only $12.2 \%$ of people with diabetes achieved all three treatment goals.

While numerous interventions to improve adherence to the recommended standards have been implemented, the challenge of providing uniformly effective diabetes care has thus far defied a simple solution. A major contributor to suboptimal care is a delivery system that too often is fragmented, lacks clinical information capabilities, often duplicates services, and is poorly designed for the delivery of chronic care. The chronic care model (CCM) includes five core elements for the provision of optimal care of patients with chronic disease: delivery system design, self-management support, decision support, clinical information systems, and community resources and policies. Redefinition of the roles of the clinic staff and promoting self-management on the part of the patient are fundamental to the successful implementation of the CCM (394). Collaborative, multidisciplinary teams are best suited to provide such care for people with chronic conditions like diabetes and to empower patients' performance of appropriate self-management. Alterations in reimbursement that reward the provision of quality care, as defined by the attainment of quality measures developed by such programs as the ADA/ National Committee for Quality Assurance Diabetes Provider Recognition Program, will also be required to achieve desired outcome goals.

In recent years, numerous health care organizations, ranging from large health care systems such as the U.S. Veteran's Administration to small private practices, have implemented strategies to improve diabetes care. Successful programs have published results showing improvement in process measures such as measurement of AlC, lipids, and blood pressure. Effects on in important intermediate outcomes, such as mean AlC for populations, have been more difficult to demonstrate (395397), although examples do exist (398402), often taking more than 1 year to manifest (394). Features of successful 
programs reported in the literature include

- Delivery of DSME: increases adherence to standard of care and educating patients on glycemic targets and improves the percentage of patients who reach goal A1C $(142,403)$

- Adoption of practice guidelines, with participation of health care professionals in the process of development: Guidelines should be readily accessible at the point of service, preferably as computerized reminders at the point of care. Guidelines should begin with a summary of their major recommendations instructing health care professionals what to do and how to do it.

- Use of checklists that mirror guidelines: successful at improving adherence to standards of care

- Systems changes: such as provision of automated reminders to health care professionals and patients and audit and feedback of process and outcome data to providers

- Quality improvement programs combining continuous quality improvement or other cycles of analysis and intervention with provider performance data

- Practice changes: such as availability of point of care testing of AlC, scheduling planned diabetes visits, clustering of dedicated diabetes visits into specific times within a primary care practice schedule, or group visits and/or visits with multiple health care professionals on a single day

- Tracking systems with either an electronic medical record or patient registry: helpful at increasing adherence to standards of care by prospectively identifying those requiring assessments and/or treatment modifications. They likely could have greater efficacy if they suggested specific therapeutic interventions to be considered for a particular patient at a particular point in time (404).

- Availability of case or (preferably) care management services (405): Nurses, pharmacists, and other nonphysician health care professionals using detailed algorithms working under the supervision of physicians have demonstrated the greatest reduction in AlC and blood pressure $(406,407)$.

Evidence suggests that these individual initiatives work best when provided as components of a multifactorial interven- tion. When practices are compared, those that address more of the CCM elements demonstrate lower AlC levels and lower cardiovascular risk scores (408). The most successful practices have an institutional priority for quality of care, involve all of the staff in their initiatives, redesign their delivery system, activate and educate their patients, and use electronic health record tools $(409,410)$.

NDEP maintains an online resource (www.betterdiabetescare.nih.gov) to help health care professionals design and implement more effective health care delivery systems for those with diabetes.

It is clear that optimal diabetes management requires an organized, systematic approach and involvement of a coordinated team of dedicated health care professionals working in an environment where quality care is a priority.

\section{References}

1. American Diabetes Asociation. Medical Management of Type 1 Diabetes. Alexandria, VA, American Diabetes Association, 2008

2. American Diabetes Asociation. Medical Management of Type 2 Diabetes. Alexandria, VA, American Diabetes Association, 2008

3. American Diabetes Association. Intensive Diabetes Management. Alexandria, VA, American Diabetes Association, 2009

4. Expert Committee on the Diagnosis and Classification of Diabetes Mellitus. Report of the Expert Committee on the Diagnosis and Classification of Diabetes Mellitus. Diabetes Care 1997;20:11831197

5. International Expert Committee. International Expert Committee report on the role of the AlC assay in the diagnosis of diabetes. Diabetes Care 2009;32:13271334

6. American Diabetes Association. Diagnosis and classification of diabetes mellitus. Diabetes Care 2010;33(Suppl. 1):S62_ S69

7. Genuth S, Alberti KG, Bennett P, Buse J, Defronzo R, Kahn R, Kitzmiller J, Knowler WC, Lebovitz H, Lernmark A, Nathan D, Palmer J, Rizza R, Saudek C, Shaw J, Steffes M, Stern M, Tuomilehto J, Zimmet P, Expert Committee on the Diagnosis and Classification of Diabetes Mellitus. Follow-up report on the diagnosis of diabetes mellitus. Diabetes Care 2003;26:3160-3167

9. Engelgau MM, Narayan KM, Herman WH. Screening for type 2 diabetes. Diabetes Care 2000;23:1563-1580

10. Gabir MM, Hanson RL, Dabelea D, Imperatore G, Roumain J, Bennett PH, Knowler WC. The 1997 American Dia- betes Association and 1999 World Health Organization criteria for hyperglycemia in the diagnosis and prediction of diabetes. Diabetes Care 2000;23: 1108-1112

11. Knowler WC, Barrett-Connor E, Fowler SE, Hamman RF, Lachin JM, Walker EA, Nathan DM, Diabetes Prevention Program Research Group. Reduction in the incidence of type 2 diabetes with lifestyle intervention or metformin. N Engl J Med 2002;346:393-403

12. Tuomilehto J, Lindström J, Eriksson JG, Valle TT, Hämäläinen H, Ilanne-Parikka $\mathrm{P}$, Keinänen-Kiukaanniemi S, Laakso M, Louheranta A, Rastas M, Salminen V, Uusitupa M, Finnish Diabetes Prevention Study Group. Prevention of type 2 diabetes mellitus by changes in lifestyle among subjects with impaired glucose tolerance. N Engl J Med 2001;344: 1343-1350

13. Pan XR, Li GW, Hu YH, Wang JX, Yang WY, An ZX, Hu ZX, Lin J, Xiao JZ, Cao HB, Liu PA, Jiang XG, Jiang YY, Wang JP, Zheng H, Zhang H, Bennett PH, Howard $\mathrm{BV}$. Effects of diet and exercise in preventing NIDDM in people with impaired glucose tolerance. The Da Qing IGT and Diabetes Study. Diabetes Care 1997;20: 537-544

14. Buchanan TA, Xiang AH, Peters RK, Kjos SL, Marroquin A, Goico J, Ochoa C, Tan S, Berkowitz K, Hodis HN, Azen SP. Preservation of pancreatic beta-cell function and prevention of type 2 diabetes by pharmacological treatment of insulin resistance in high-risk hispanic women. Diabetes 2002;51:2796-2803

15. Chiasson JL, Josse RG, Gomis R, Hanefeld M, Karasik A, Laakso M, STOP-NIDDM Trail Research Group. Acarbose for prevention of type 2 diabetes mellitus: the STOP-NIDDM randomised trial. Lancet 2002;359:2072-2077

16. DREAM (Diabetes REduction Assessment with ramipril and rosiglitazone Medication) Trial Investigators, Gerstein HC, Yusuf S, Bosch J, Pogue J, Sheridan P, Dinccag N, Hanefeld M, Hoogwerf B, Laakso M, Mohan V, Shaw J, Zinman B, Holman RR. Effect of rosiglitazone on the frequency of diabetes in patients with impaired glucose tolerance or impaired fasting glucose: a randomised controlled trial. Lancet 2006;368:10961105

17. Ramachandran A, Snehalatha C, Mary S, Mukesh B, Bhaskar AD, Vijay V, Indian Diabetes Prevention Programme (IDPP). The Indian Diabetes Prevention Programme shows that lifestyle modification and metformin prevent type 2 diabetes in Asian Indian subjects with impaired glucose tolerance (IDPP-1). Diabetologia 2006;49:289-297

18. Johnson SL, Tabaei BP, Herman WH. The efficacy and cost of alternative strat- 
egies for systematic screening for type 2 diabetes in the U.S. population 45-74 years of age. Diabetes Care 2005;28: 307-311

19. Harris R, Donahue K, Rathore SS, Frame $\mathrm{P}$, Woolf SH, Lohr KN. Screening adults for type 2 diabetes: a review of the evidence for the U.S. Preventive Services Task Force. Ann Intern Med 2003;138: 215-229

20. U.S. Preventive Services Task Force. S. Preventive Services Task Force. S. Preventive Services Task Force: Screening for type 2 diabetes mellitus in adults: recommendations and rationale. Ann Intern Med 2003;138:212-214

21. Writing Group for the SEARCH for Diabetes in Youth Study Group, Dabelea D, Bell RA, D'Agostino RB Jr, Imperatore G, Johansen JM, Linder B, Liu LL, Loots B, Marcovina S, Mayer-Davis EJ, Pettitt DJ, Waitzfelder B. Incidence of diabetes in youth in the United States. JAMA 2007; 297:2716-2724

22. SEARCH for Diabetes in Youth Study Group, Liese AD, D'Agostino RB Jr, Hamman RF, Kilgo PD, Lawrence JM, Liu LL, Loots B, Linder B, Marcovina S, Rodriguez B, Standiford D, Williams DE. The burden of diabetes mellitus among US youth: prevalence estimates from the SEARCH for Diabetes in Youth Study. Pediatrics 2006;118:1510-1518

23. American Diabetes Association: Type 2 diabetes in children and adolescents (Consensus Statement). Diabetes Care 2000;23:381-389

24. Lawrence JM, Contreras R, Chen W, Sacks DA. Trends in the prevalence of preexisting diabetes and gestational diabetes mellitus among a racially/ethnically diverse population of pregnant women, 1999-2005. Diabetes Care 2008;31:899-904

25. American Diabetes Association: Gestational diabetes mellitus (Position Statement). Diabetes Care 2004;27(Suppl. 1):S88-S90

26. HAPO Study Cooperative Research Group, Metzger BE, Lowe LP, Dyer AR, Trimble ER, Chaovarindr U, Coustan DR, Hadden DR, McCance DR, Hod M, McIntyre HD, Oats JJ, Persson B, Rogers MS, Sacks DA. Hyperglycemia and adverse pregnancy outcomes. N Engl J Med 2008;358:1991-2002

27. Landon MB, Spong CY, Thom E, Carpenter MW, Ramin SM, Casey B, Wapner RJ, Varner MW, Rouse DJ, Thorp JM Jr, Sciscione A, Catalano P, Harper M, Saade G, Lain KY, Sorokin Y, Peaceman AM, Tolosa JE, Anderson GB, Eunice Kennedy Shriver National Institute of Child Health and Human Development Maternal-Fetal Medicine Units Network. A multicenter, randomized trial of treatment for mild gestational diabetes. N Engl J Med 2009;361:1339-1348
28. Kim C, Newton KM, Knopp RH. Gestational diabetes and the incidence of type 2 diabetes: a systematic review. Diabetes Care 2002;25:1862-1868

29. Lindström J, Ilanne-Parikka P, Peltonen M, Aunola S, Eriksson JG, Hemiö K, Hämäläinen $\mathrm{H}$, Härkönen $\mathrm{P}$, KeinänenKiukaanniemi S, Laakso M, Louheranta A, Mannelin M, Paturi M, Sundvall J, Valle TT, Uusitupa M, Tuomilehto J, Finnish Diabetes Prevention Study Group. Sustained reduction in the incidence of type 2 diabetes by lifestyle intervention: follow-up of the Finnish Diabetes Prevention Study. Lancet 2006; 368:1673-1679

30. Li G, Zhang P, Wang J, Gregg EW, Yang W, Gong Q, Li H, Li H, Jiang Y, An Y, Shuai Y, Zhang B, Zhang J, Thompson TJ, Gerzoff RB, Roglic G, Hu Y, Bennett $\mathrm{PH}$. The long-term effect of lifestyle interventions to prevent diabetes in the China Da Qing Diabetes Prevention Study: a 20-year follow-up study. Lancet 2008;371:1783-1789

31. Kosaka K, Noda M, Kuzuya T. Prevention of type 2 diabetes by lifestyle intervention: a Japanese trial in IGT males. Diabetes Res Clin Pract 2005;67:152162

32. Torgerson JS, Hauptman J, Boldrin MN, Sjöström L. XENical in the prevention of diabetes in obese subjects (XENDOS) study: a randomized study of orlistat as an adjunct to lifestyle changes for the prevention of type 2 diabetes in obese patients. Diabetes Care 2004:27:155161

33. Kawamori R, Tajima N, Iwamoto $\mathrm{Y}$, Kashiwagi A, Shimamoto K, Kaku K, Voglibose Ph-3 Study Group. Voglibose for prevention of type 2 diabetes mellitus: a randomised, double-blind trial in Japanese individuals with impaired glucose tolerance. Lancet 2009; 373:1607-1614

34. DeFronzo RA, for ACT NOW Study Group. ACTos NOW Study for the Prevention of Diabetes (ACT NOW) Study. Late-breaking abstract presented at 68th Annual Scientific Sessions of the American Diabetes Association, 6 June 2008, San Francisco, CA

35. Gerstein HC. Point: If it is important to prevent type 2 diabetes, it is important to consider all proven therapies within a comprehensive approach. Diabetes Care 2007;30:432-434

36. Nathan DM, Davidson MB, DeFronzo RA, Heine RJ, Henry RR, Pratley R, Zinman B, American Diabetes Association. Impaired fasting glucose and impaired glucose tolerance: implications for care. Diabetes Care 2007;30:753-759

37. American Diabetes Association: Consensus statement on self-monitoring of blood glucose. Diabetes Care 1987;10: 95-99
38. American Diabetes Association: Selfmonitoring of blood glucose. Diabetes Care 1994;17:81-86

39. Welschen LM, Bloemendal E, Nijpels G, Dekker JM, Heine RJ, Stalman WA, Bouter LM. Self-monitoring of blood glucose in patients with type 2 diabetes who are not using insulin: a systematic review. Diabetes Care 2005;28:15101517

40. Farmer A, Wade A, Goyder E, Yudkin $\mathrm{P}$, French D, Craven A, Holman R, Kinmonth AL, Neil A. Impact of self monitoring of blood glucose in the management of patients with non-insulin treated diabetes: open parallel group randomised trial. BMJ 2007;335:132

41. O'Kane MJ, Bunting B, Copeland M, Coates VE, ESMON study group. Efficacy of self monitoring of blood glucose in patients with newly diagnosed type 2 diabetes (ESMON study): randomised controlled trial. BMJ 2008;336:11741177

42. Simon J, Gray A, Clarke P, Wade A, Neil A, Farmer A, Diabetes Glycaemic Education and Monitoring Trial Group. Cost effectiveness of self monitoring of blood glucose in patients with non-insulin treated type 2 diabetes: economic evaluation of data from the DiGEM trial. BMJ 2008;336:1177-1180

43. Sacks DB, Bruns DE, Goldstein DE, Maclaren NK, McDonald JM, Parrott M. Guidelines and recommendations for laboratory analysis in the diagnosis and management of diabetes mellitus. Clin Chem 2002:48:436-472

44. Juvenile Diabetes Research Foundation Continuous Glucose Monitoring Study Group, Tamborlane WV, Beck RW, Bode BW, Buckingham B, Chase HP, Clemons R, Fiallo-Scharer R, Fox LA, Gilliam LK, Hirsch IB, Huang ES, Kollman C, Kowalski AJ, Laffel L, Lawrence JM, Lee J, Mauras N, O'Grady M, Ruedy KJ, Tansey M, Tsalikian E, Weinzimer S, Wilson DM, Wolpert H, Wysocki T, Xing D: Continuous glucose monitoring and intensive treatment of type 1 diabetes. N Engl J Med 2008; 359:1464-1476

45. Juvenile Diabetes Research Foundation Continuous Glucose Monitoring Study Group: The effect of continuous glucose monitoring in well-controlled type 1 diabetes. Diabetes Care 2009;32:13781383

46. Stratton IM, Adler AI, Neil HA, Matthews DR, Manley SE, Cull CA, Hadden D, Turner RC, Holman RR. Association of glycaemia with macrovascular and microvascular complications of type 2 diabetes (UKPDS 35): prospective observational study. BMJ 2000;321:405412

47. Cagliero E, Levina EV, Nathan DM. Immediate feedback of HbAlc levels improves glycemic control in type 1 and 
insulin-treated type 2 diabetic patients. Diabetes Care 1999;22:1785-1789

48. Miller CD, Barnes CS, Phillips LS, Ziemer DC, Gallina DL, Cook CB, Maryman SD, El-Kebbi IM. Rapid Alc availability improves clinical decisionmaking in an urban primary care clinic. Diabetes Care 2003;26:1158-1163

49. Nathan DM, Kuenen J, Borg R, Zheng H, Schoenfeld D, Heine RJ, Alc-Derived Average Glucose Study Group. Translating the $\mathrm{AlC}$ assay into estimated average glucose values. Diabetes Care 2008;31: 1473-1478

50. Rohlfing CL, Wiedmeyer HM, Little RR, England JD, Tennill A, Goldstein DE. Defining the relationship between plasma glucose and $\mathrm{HbA}(1 \mathrm{c})$ : analysis of glucose profiles and $\mathrm{HbA}(1 \mathrm{c})$ in the Diabetes Control and Complications Trial. Diabetes Care 2002;25:275-278

51. Diabetes Research in Children Network (DirecNet) Study Group, Wilson DM, Kollman. Relationship of AlC to glucose concentrations in children with type 1 diabetes: assessments by high-frequency glucose determinations by sensors. Diabetes Care 2008;31:381-385

52. Skyler JS, Bergenstal R, Bonow RO, Buse J, Deedwania P, Gale EA, Howard BV, Kirkman MS, Kosiborod M, Reaven P, Sherwin RS, American Diabetes Association, American College of Cardiology Foundation, American Heart Association. Intensive glycemic control and the prevention of cardiovascular events: implications of the ACCORD, ADVANCE, and VA diabetes trials: a position statement of the American Diabetes Association and a scientific statement of the American College of Cardiology Foundation and the American Heart Association. Diabetes Care 2009;32:187-192

53. The effect of intensive treatment of diabetes on the development and progression of long-term complications in insulin-dependent diabetes mellitus. The Diabetes Control and Complications Trial Research Group. N Engl J Med 1993;329:977-986

54. Retinopathy and nephropathy in patients with type 1 diabetes four years after a trial of intensive therapy. The Diabetes Control and Complications Trial/Epidemiology of Diabetes Interventions and Complications Research Group. N Engl J Med 2000;342:381-389

55. Martin CL, Albers J, Herman WH Cleary P, Waberski B, Greene DA, Stevens MJ, Feldman EL, DCCT/EDIC Research Group. Neuropathy among the diabetes control and complications trial cohort 8 years after trial completion. Diabetes Care 2006;29:340-344

56. Ohkubo Y, Kishikawa H, Araki E, Miyata T, Isami S, Motoyoshi S, Kojima Y, Furuyoshi $\mathrm{N}$, Shichiri $\mathrm{M}$. Intensive insulin therapy prevents the progression of dia- betic microvascular complications in Japanese patients with non-insulin-dependent diabetes mellitus: a randomized prospective 6-year study. Diabetes Res Clin Pract 1995;28:103-117

57. Effect of intensive blood-glucose control with metformin on complications in overweight patients with type 2 diabetes (UKPDS 34): UK Prospective Diabetes Study (UKPDS) Group. Lancet 1998; 352:854-865

58. Intensive blood-glucose control with sulphonylureas or insulin compared with conventional treatment and risk of complications in patients with type 2 diabetes (UKPDS 33): UK Prospective Diabetes Study (UKPDS) Group. Lancet 1998;352:837-853

59. Holman RR, Paul SK, Bethel MA, Matthews DR, Neil HA: 10-Year follow-up of intensive glucose control in type 2 diabetes. N Engl J Med 2008;359:15771589

60. Duckworth W, Abraira C, Moritz T, Reda D, Emanuele N, Reaven PD, Zieve FJ, Marks J, Davis SN, Hayward R, Warren SR, Goldman S, McCarren M, Vitek ME, Henderson WG, Huang GD, VADT Investigators. Glucose control and vascular complications in veterans with type 2 diabetes. N Engl J Med 2009;360: 129-139

61. Moritz T, Duckworth W, Abraira C. Veterans Affairs diabetes trial-corrections. N Engl J Med 2009;361:1024-1025

62. Lawson ML, Gerstein HC, Tsui E, Zinman B. Effect of intensive therapy on early macrovascular disease in young individuals with type 1 diabetes: A systematic review and meta-analysis. Diabetes Care 1999;22(Suppl. 2):B35-B39

63. ADVANCE Collaborative Group, Patel A, MacMahon S, Chalmers J, Neal B, Billot L, Woodward M, Marre M, Cooper M, Glasziou P, Grobbee D, Hamet P, Harrap S, Heller S, Liu L, Mancia G, Mogensen CE, Pan C, Poulter N, Rodgers A, Williams B, Bompoint S, de Galan BE, Joshi R, Travert F. Intensive blood glucose control and vascular outcomes in patients with type 2 diabetes. $\mathrm{N}$ Engl J Med 2008;358:2560-2572

64. Selvin E, Marinopoulos S, Berkenblit G, Rami T, Brancati FL, Powe NR, Golden SH. Meta-analysis: glycosylated hemoglobin and cardiovascular disease in diabetes mellitus. Ann Intern Med 2004; 141:421-431

65. Stettler C, Allemann S, Jüni P, Cull CA, Holman RR, Egger M, Krähenbühl S, Diem P. Glycemic control and macrovascular disease in types 1 and 2 diabetes mellitus: Meta-analysis of randomized trials. Am Heart J 2006;152:27-38

66. Nathan DM, Cleary PA, Backlund JY, Genuth SM, Lachin JM, Orchard TJ, Raskin P, Zinman B, Diabetes Control and Complications Trial/Epidemiology of Diabetes Interventions and Complications (DCCT/EDIC) Study Research Group. Intensive diabetes treatment and cardiovascular disease in patients with type 1 diabetes. N Engl J Med 2005;353: 2643-2653

67. Diabetes Control and Complications Trial/Epidemiology of Diabetes Interventions and Complications (DCCT/ EDIC) Research Group, Nathan DM, Zinman B, Cleary PA, Backlund JY, Genuth S, Miller R, Orchard TJ. Modernday clinical course of type 1 diabetes mellitus after 30 years' duration: the diabetes control and complications trial/ epidemiology of diabetes interventions and complications and Pittsburgh epidemiology of diabetes complications experience (1983-2005). Arch Intern Med 2009;169:1307-1316

68. Action to Control Cardiovascular Risk in Diabetes Study Group, Gerstein HC, Miller ME, Byington RP, Goff DC Jr, Bigger JT, Buse JB, Cushman WC, Genuth $\mathrm{S}$, Ismail-Beigi F, Grimm RH Jr, Probstfield JL, Simons-Morton DG, Friedewald WT. Effects of intensive glucose lowering in type 2 diabetes. N Engl J Med 2008;358:2545-2559

69. Duckworth W. VADT Results. Latebreaking abstract presented at 68th Annual Scientific Sessions of the American Diabetes Association, 6 June 2008, San Francisco, CA

70. Reaven PD, Moritz TE, Schwenke DC, Anderson RJ, Criqui M, Detrano R, Emanuele N, Kayshap M, Marks J, Mudaliar S, Rao RH, Shah JH, Goldman S, Reda DJ, McCarren M, Abraira C, Duckworth W: Intensive glucose lowering therapy reduces cardiovascular disease events in VADT participants with lower calcified coronary atherosclerosis. Diabetes 2009;59:2642-2648

71. Turnbull FM, Abraira C, Anderson RJ, Byington RP, Chalmers JP, Duckworth WC, Evans GW, Gerstein HC, Holman RR, Moritz TE, Neal BC, Ninomiya T, Patel AA, Paul SK, Travert F, Woodward $\mathrm{M}$ : Intensive glucose control and macrovascular outcomes in type 2 diabetes. Diabetologia 2009;52:2288-2298

72. American Diabetes Association: Postprandial blood glucose (Consensus Statement). Diabetes Care 2001;24:775778

73. Ceriello A, Taboga C, Tonutti L, Quagliaro L, Piconi L, Bais B, Da Ros R, Motz E. Evidence for an independent and cumulative effect of postprandial hypertriglyceridemia and hyperglycemia on endothelial dysfunction and oxidative stress generation: effects of short- and long-term simvastatin treatment. Circulation 2002;106:1211-1218

74. Raz I, Wilson PW, Strojek K, Kowalska I, Bozikov V, Gitt AK, Jermendy G, Campaigne BN, Kerr L, Milicevic Z, Jacober 
SJ. Effects of prandial versus fasting glycemia on cardiovascular outcomes in type 2 diabetes: the HEART2D trial. Diabetes Care 2009;32:381-386

75. Metzger BE, Buchanan TA, Coustan DR, de Leiva A, Dunger DB, Hadden DR, Hod M, Kitzmiller JL, Kjos SL, Oats JN, Pettitt DJ, Sacks DA, Zoupas C. Summary and recommendations of the Fifth International Workshop-Conference on Gestational Diabetes Mellitus. Diabetes Care 2007;30(Suppl. 2):S251-S260

76. Kitzmiller JL, Block JM, Brown FM, Catalano PM, Conway DL, Coustan DR, Gunderson EP, Herman WH, Hoffman $\mathrm{LD}$, Inturrisi M, Jovanovic LB, Kjos SI, Knopp RH, Montoro MN, Ogata ES, Paramsothy P, Reader DM, Rosenn BM, Thomas AM, Kirkman MS. Managing preexisting diabetes for pregnancy: summary of evidence and consensus recommendations for care. Diabetes Care 2008;31:1060-1079

77. DeWitt DE, Hirsch IB. Outpatient insulin therapy in type 1 and type 2 diabetes mellitus: scientific review. JAMA 2003; 289:2254-2264

78. Rosenstock J, Dailey G, Massi-Benedetti M, Fritsche A, Lin Z, Salzman A. Reduced hypoglycemia risk with insulin glargine: a meta-analysis comparing insulin glargine with human NPH insulin in type 2 diabetes. Diabetes Care 2005; 28:950-955

79. Mooradian AD, Bernbaum M, Albert SG. Narrative review: a rational approach to starting insulin therapy. Ann Intern Med 2006; 145:125-134

80. Nathan DM, Buse JB, Davidson MB, Heine RJ, Holman RR, Sherwin R, Zinman B. Management of hyperglycemia in type 2 diabetes: a consensus algorithm for the initiation and adjustment of therapy: a consensus statement from the American Diabetes Association and the European Association for the Study of Diabetes. Diabetes Care 2006;29:19631972

81. Nathan DM, Buse JB, Davidson MB, Ferrannini E, Holman RR, Sherwin R, Zinman B, American Diabetes Association, European Association for Study of Diabetes. Medical management of hyperglycemia in type 2 diabetes: a consensus algorithm for the initiation and adjustment of therapy: a consensus statement of the American Diabetes Association and the European Association for the Study of Diabetes. Diabetes Care 2009; 32:193-203

82. Bantle JP, Wylie-Rosett J, Albright AL, Apovian CM, Clark NG, Franz MJ, Hoogwerf BJ, Lichtenstein AH, MayerDavis E, Mooradian AD, Wheeler ML. Nutrition recommendations and interventions for diabetes-2006. Diabetes Care 2006;29:2140-2157

83. DAFNE Study Group. Training in flexi- ble, intensive insulin management to enable dietary freedom in people with type 1 diabetes: dose adjustment for normal eating (DAFNE) randomised controlled trial. BMJ 2002;325:746

84. Franz MJ, Monk A, Barry B, McClain K, Weaver T, Cooper N, Upham P, Bergenstal R, Mazze RS. Effectiveness of medical nutrition therapy provided by dietitians in the management of non-insulin-dependent diabetes mellitus: a randomized, controlled clinical trial. J Am Diet Assoc 1995;95:1009-1017

85. Goldhaber-Fiebert JD, Goldhaber-Fiebert SN, Tristán ML, Nathan DM. Randomized controlled community-based nutrition and exercise intervention improves glycemia and cardiovascular risk factors in type 2 diabetic patients in rural Costa Rica. Diabetes Care 2003;26:24-29

86. Lemon CC, Lacey K, Lohse B, Hubacher DO, Klawitter B, Palta M. Outcomes monitoring of health, behavior, and quality of life after nutrition intervention in adults with type 2 diabetes. J Am Diet Assoc 2004;104:1805-1815

87. Miller CK, Edwards L, Kissling G, Sanville L. Nutrition education improves metabolic outcomes among older adults with diabetes mellitus: results from a randomized controlled trial. Prev Med 2002;34:252-259

88. Wilson C, Brown T, Acton K, Gilliland S. Effects of clinical nutrition education and educator discipline on glycemic control outcomes in the Indian health service. Diabetes Care 2003;26:2500 2504

89. Graber AL, Elasy TA, Quinn D, Wolff K, Brown A. Improving glycemic control in adults with diabetes mellitus: shared responsibility in primary care practices. South Med J 2002;95:684-690

90. Gaetke LM, Stuart MA, Truszczynska H. A single nutrition counseling session with a registered dietitian improves short-term clinical outcomes for rural Kentucky patients with chronic diseases. J Am Diet Assoc 2006;106:109-112

91. Yu-Poth S, Zhao G, Etherton T, Naglak M, Jonnalagadda S, Kris-Etherton PM. Effects of the National Cholesterol Education Program's Step I and Step II dietary intervention programs on cardiovascular disease risk factors: a metaanalysis. Am J Clin Nutr 1999;69:632646

92. Van Horn L, McCoin M, Kris-Etherton PM, Burke F, Carson JA, Champagne CM, Karmally W, Sikand G. The evidence for dietary prevention and treatment of cardiovascular disease. J Am Diet Assoc 2008;108:287-331

93. Appel LJ, Moore TJ, Obarzanek E, Vollmer WM, Svetkey LP, Sacks FM, Bray GA, Vogt TM, Cutler JA, Windhauser MM, Lin PH, Karanja N. A clinical trial of the effects of dietary pat- terns on blood pressure. DASH Collaborative Research Group. N Engl J Med 1997;336:1117-1124

94. Norris SL, Zhang X, Avenell A, Gregg E, Bowman B, Schmid CH, Lau J. Longterm effectiveness of weight-loss interventions in adults with pre-diabetes: a review. Am J Prev Med 2005;28:126139

95. Klein S, Sheard NF, Pi-Sunyer X, Daly A, Wylie-Rosett J, Kulkarni K, Clark NG, American Diabetes Association, North American Association for the Study of Obesity, American Society for Clinical Nutrition. Weight management through lifestyle modification for the prevention and management of type 2 diabetes: rationale and strategies: a statement of the American Diabetes Association, the North American Association for the Study of Obesity, and the American Society for Clinical Nutrition. Diabetes Care 2004:27:2067-2073

96. Norris SL, Zhang X, Avenell A, Gregg E, Schmid CH, Kim C, Lau J. Efficacy of pharmacotherapy for weight loss in adults with type 2 diabetes mellitus: a meta-analysis. Arch Intern Med 2004; 164:1395-1404

97. Wolf AM, Conaway MR, Crowther JQ, Hazen KY, L Nadler J, Oneida B, Bovbjerg VE, Improving Control with Activity and Nutrition (ICAN) Study. Translating lifestyle intervention to practice in obese patients with type 2 diabetes: Improving Control with Activity and Nutrition (ICAN) study. Diabetes Care 2004; 27:1570-1576

98. Manning RM, Jung RT, Leese GP, Newton RW. The comparison of four weight reduction strategies aimed at overweight patients with diabetes mellitus: fouryearfollow-up.DiabetMed1998;15:497502

99. Shai I, Schwarzfuchs D, Henkin Y, Shahar DR, Witkow S, Greenberg I, Golan R, Fraser D, Bolotin A, Vardi H, TangiRozental O, Zuk-Ramot R, Sarusi B, Brickner D, Schwartz Z, Sheiner E, Marko R, Katorza E, Thiery J, Fiedler GM, Blüher M, Stumvoll M, Stampfer MJ, Dietary Intervention Randomized Controlled Trial (DIRECT) Group. Weight loss with a low-carbohydrate, Mediterranean, or low-fat diet. N Engl J Med 2008;359:229-241

100. Franz MJ, VanWormer JJ, Crain AL, Boucher JL, Histon T, Caplan W, Bowman JD, Pronk NP. Weight-loss outcomes: a systematic review and metaanalysis of weight-loss clinical trials with a minimum 1-year follow-up. J Am Diet Assoc 2007; 107:1755-1767

101. Look AHEAD Research Group, Pi-Sunyer X, Blackburn G, Brancati FL, Bray GA, Bright R, Clark JM, Curtis JM, Espeland MA, Foreyt JP, Graves K, Haffner SM, Harrison B, Hill JO, Horton ES, Ja- 
kicic J, Jeffery RW, Johnson KC, Kahn S, Kelley DE, Kitabchi AE, Knowler WC, Lewis CE, Maschak-Carey BJ, Montgomery B, Nathan DM, Patricio J, Peters A, Redmon JB, Reeves RS, Ryan DH, Safford M, Van Dorsten B, Wadden TA, Wagenknecht L, Wesche-Thobaben J, Wing RR, Yanovski SZ. Reduction in weight and cardiovascular disease risk factors in individuals with type 2 diabetes: one-year results of the look AHEAD trial. Diabetes Care 2007;30:1374-1383

102. Foster GD, Wyatt HR, Hill JO, McGuckin BG, Brill C, Mohammed BS, Szapary PO, Rader DJ, Edman JS, Klein $S$. A randomized trial of a low-carbohydrate diet for obesity. N Engl J Med 2003;348:2082-2090

103. Stern L, Iqbal N, Seshadri P, Chicano KL, Daily DA, McGrory J, Williams M, Gracely EJ, Samaha FF. The effects of low-carbohydrate versus conventional weight loss diets in severely obese adults: one-year follow-up of a randomized trial. Ann Intern Med 2004;140: 778-785

104. Gardner CD, Kiazand A, Alhassan S, Kim S, Stafford RS, Balise RR, Kraemer HC, King AC. Comparison of the Atkins, Zone, Ornish, and LEARN diets for change in weight and related risk factors among overweight premenopausal women. JAMA 2007;297:969977

105. Nordmann AJ, Nordmann A, Briel M, Keller U, Yancy WS, Jr, Brehm BJ, Bucher HC. Effects of low-carbohydrate vs low-fat diets on weight loss and cardiovascular risk factors: a meta-analysis of randomized controlled trials. Arch Intern Med 2006;166:285-293

106. Institute of Medicine: DIetary Reference Intakes: Energy, Carbohydrate, Fiber, Fat, Fatty Acids, Cholesterol, Protein, and Amino Acids. Washington, DC, National Academies Press, 2002

107. Barnard ND, Cohen J, Jenkins DJ, TurnerMcGrievy G, Gloede L, Jaster B, Seidl K, Green AA, Talpers S. A low-fat vegan diet improves glycemic control and cardiovascular risk factors in a randomized clinical trial in individuals with type 2 diabetes. Diabetes Care 2006;29:17771783

108. Turner-McGrievy GM, Barnard ND, Cohen J, Jenkins DJ, Gloede L, Green AA. Changes in nutrient intake and dietary quality among participants with type 2 diabetes following a low-fat vegan diet or a conventional diabetes diet for 22 weeks. J Am Diet Assoc 2008;108: 1636-1645

109. Franz MJ, Bantle JP, Beebe CA, Brunzell JD, Chiasson JL, Garg A, Holzmeister LA, Hoogwerf B, Mayer-Davis E, Mooradian AD, Purnell JQ, Wheeler M. Evidence-based nutrition principles and recommendations for the treatment and prevention of diabetes and related complications. Diabetes Care 2002;25:148198

110. Buchwald H, Estok R, Fahrbach K, Banel $\mathrm{D}$, Jensen $\mathrm{MD}$, Pories WJ, Bantle JP, Sledge I. Weight and type 2 diabetes after bariatric surgery: systematic review and meta-analysis. Am J Med 2009; 122 : $248-256$

111. Dixon JB, O’Brien PE, Playfair J, Chapman L, Schachter LM, Skinner S, Proietto J, Bailey M, Anderson M. Adjustable gastric banding and conventional therapy for type 2 diabetes: a randomized controlled trial. JAMA 2008;299:316323

112. Buchwald H, Estok R, Fahrbach K, Banel $\mathrm{D}$, Sledge I. Trends in mortality in bariatric surgery: a systematic review and meta-analysis. Surgery 2007;142:621632

113. Sjöström L, Narbro K, Sjöström CD, Karason K, Larsson B, Wedel H, Lystig T, Sullivan M, Bouchard C, Carlsson B, Bengtsson C, Dahlgren S, Gummesson A, Jacobson P, Karlsson J, Lindroos AK, Lönroth H, Näslund I, Olbers T, Stenlöf K, Torgerson J, Agren G, Carlsson LM, Swedish Obese Subjects Study. Effects of bariatric surgery on mortality in Swedish obese subjects. N Engl J Med 2007;357: 741-752

114. Piette JD, Glasgow RE: Sttategies for improving behavioral and health outcomes among people with diabetes: self management education. In Evidence-Based Diabetes Care. Gerstein HC, Hayes RB, Eds. Ontario, Canada, BC Decker, 2000

115. Norris SL, Engelgau MM, Narayan KM. Effectiveness of self-management training in type 2 diabetes: a systematic review of randomized controlled trials. Diabetes Care 2001:24:561-587

116. Norris SL, Lau J, Smith SJ, Schmid CH, Engelgau MM. Self-management education for adults with type 2 diabetes: a meta-analysis of the effect on glycemic control. Diabetes Care 2002;25:1159_ 1171

117. Gary TL, Genkinger JM, Guallar E, Peyrot M, Brancati FL. Meta-analysis of randomized educational and behavioral interventions in type 2 diabetes. Diabetes Educ 2003;29:488-501

118. Steed L, Cooke D, Newman S. A systematic review of psychosocial outcomes following education, self-management and psychological interventions in diabetes mellitus. Patient Educ Couns 2003:51:5-15

119. Ellis SE, Speroff T, Dittus RS, Brown A, Pichert JW, Elasy TA. Diabetes patient education: a meta-analysis and meta-regression. Patient Educ Couns 2004;52: 97-105

120. Warsi A, Wang PS, LaValley MP, Avorn J, Solomon DH. Self-management education programs in chronic disease: a systematic review and methodological critique of the literature. Arch Intern Med 2004;164:1641-1649

121. Funnell MM, Brown TL, Childs BP, Haas LB, Hosey GM, Jensen B, Maryniuk M, Peyrot M, Piette JD, Reader D, Siminerio LM, Weinger K, Weiss MA. National standards for diabetes self-management education. Diabetes Care 2007;30: 1630-1637

122. Mulcahy K, Maryniuk M, Peeples M, Peyrot M, Tomky D, Weaver T, Yarborough P. Diabetes self-management education core outcomes measures. Diabetes Educ 29:768- 2003;84:787

123. Glasgow RE, Peeples M, Skovlund SE. Where is the patient in diabetes performance measures? The case for including patient-centered and self-management measures. Diabetes Care 2008;31:10461050

124. Barker JM, Goehrig SH, Barriga K, Hoffman M, Slover R, Eisenbarth GS, Norris JM, Klingensmith GJ, Rewers M, DAISY study. Clinical characteristics of children diagnosed with type 1 diabetes through intensive screening and followup. Diabetes Care 2004;27:1399-1404

125. Cochran J, Conn VS. Meta-analysis of quality of life outcomes following diabetes self-management training. Diabetes Educ 2008;34:815-823

126. Fisher EB, Thorpe CT, Devellis BM, Devellis RF. Healthy coping, negative emotions, and diabetes management: a systematic review and appraisal. Diabetes Educ 2007;33:1080-1103

127. Robbins JM, Thatcher GE, Webb DA, Valdmanis VG. Nutritionist visits, diabetes classes, and hospitalization rates and charges: the Urban Diabetes Study. Diabetes Care 2008;31:655-660

128. Renders CM, Valk GD, Griffin SJ, Wagner EH, Eijk Van JT, Assendelft WJ. Interventions to improve the management of diabetes in primary care, outpatient, and community settings: a systematic review. Diabetes Care 2001;24:18211833

129. Polonsky WH, Earles J, Smith S, Pease DJ, Macmillan M, Christensen R, Taylor T, Dickert J, Jackson RA. Integrating medical management with diabetes selfmanagement training: a randomized control trial of the Diabetes Outpatient Intensive Treatment program. Diabetes Care 2003;26:3048-3053

130. Anderson RM, Funnell MM, Nwankwo R, Gillard ML, Oh M, Fitzgerald JT. Evaluating a problem-based empowerment program for African Americans with diabetes: results of a randomized controlled trial. Ethn Dis 2005;15: 671-678

131. Brown SA, Blozis SA, Kouzekanani K, Garcia AA, Winchell M, Hanis CL. Dosage effects of diabetes self-management education for Mexican Americans: the 
Starr County Border Health Initiative. Diabetes Care 2005;28:527-532

132. Glazier RH, Bajcar J, Kennie NR, Willson K. A systematic review of interventions to improve diabetes care in socially disadvantaged populations. Diabetes Care 2006;29:1675-1688

133. Sarkisian CA, Brown AF, Norris KC, Wintz RL, Mangione CM. A systematic review of diabetes self-care interventions for older, African American, or Latino adults. Diabetes Educ 2003;29:467-479

134. Chodosh J, Morton SC, Mojica W, Maglione M, Suttorp MJ, Hilton L, Rhodes $\mathrm{S}$, Shekelle P. Meta-analysis: chronic disease self-management programs for older adults. Ann Intern Med 2005; 143 : 427-438

135. Peyrot M, Rubin RR. Behavioral and psychosocial interventions in diabetes: a conceptual review. Diabetes Care 2007; 30:2433-2440

136. Rickheim PL, Weaver TW, Flader JL, Kendall DM. Assessment of group versus individual diabetes education: a randomized study. Diabetes Care 2002;25: 269-274

137. Trento M, Passera P, Borgo E, Tomalino M, Bajardi M, Cavallo F, Porta M. A 5-year randomized controlled study of learning, problem solving ability, and quality of life modifications in people with type 2 diabetes managed by group care. Diabetes Care 2004;27:670-675

138. Deakin T, McShane CE, Cade JE, Williams RD: Group based training for selfmanagement strategies in people with type 2 diabetes mellitus. Cochrane Database Syst Rev CD003417, 2005

139. Heisler, M. Building Peer Support Programs to Manage Chronic Disease: Seven Models for Success. Oakland, CA, California Health Care Foundation, 2006

140. Foster G, Taylor SJ, Eldridge SE, Ramsay J, Griffiths CJ: Self-management education programmes by lay leaders for people with chronic conditions. Cochrane Database Syst Rev CD005108, 2007

141. Norris SL, Chowdhury FM, Van Le K, Horsley T, Brownstein JN, Zhang X, Jack L Jr, Satterfield DW. Effectiveness of community health workers in the care of persons with diabetes. Diabet Med 2006;23:544-556

142. Duncan I, Birkmeyer C, Coughlin S, Li $\mathrm{QE}$, Sherr D, Boren S. Assessing the value of diabetes education. Diabetes Educ 2009:35:752-760

143. Sigal RJ, Kenny GP, Wasserman DH, Castaneda-Sceppa C. Physical activity/ exercise and type 2 diabetes. Diabetes Care 2004;27:2518-2539

144. Wasserman DH, Zinman B. Exercise in individuals with IDDM. Diabetes Care 1994;17:924-937

145. Boulé NG, Haddad E, Kenny GP, Wells GA, Sigal RJ. Effects of exercise on glycemic control and body mass in type 2 diabetes mellitus: a meta-analysis of controlled clinicaltrials.JAMA2001;286: 1218-1227

146. Boulé NG, Kenny GP, Haddad E, Wells GA, Sigal RJ. Meta-analysis of the effect of structured exercise training on cardiorespiratory fitness in Type 2 diabetes mellitus. Diabetologia 2003;46:10711081

147. U.S. Department of Health and Human Services. 2008 Physical Activity Guidelines for Americans. Atlanta, GA, Centers for Disease Control and Prevention, 2008

148. Cauza E, Hanusch-Enserer U, Strasser B, Ludvik B, Metz-Schimmerl S, Pacini G, Wagner O, Georg P, Prager R, Kostner K, Dunky A, Haber P. The relative benefits of endurance and strength training on the metabolic factors and muscle function of people with type 2 diabetes mellitus. Arch Phys Med Rehabil 2005;86: 1527-1533

149. Dunstan DW, Daly RM, Owen N, Jolley D, De Courten M, Shaw J, Zimmet P. High-intensity resistance training improves glycemic control in older patients with type 2 diabetes. Diabetes Care 2002;25:1729-1736

150. Castaneda C, Layne JE, Munoz-Orians L, Gordon PL, Walsmith J, Foldvari M, Roubenoff R, Tucker KL, Nelson ME. A randomized controlled trial of resistance exercise training to improve glycemic control in older adults with type 2 diabetes. Diabetes Care 2002;25:23352341

151. Sigal RJ, Kenny GP, Boulé NG, Wells GA, Prud'homme D, Fortier M, Reid RD, Tulloch H, Coyle D, Phillips P, Jennings A, Jaffey J. Effects of aerobic training, resistance training, or both on glycemic control in type 2 diabetes: a randomized trial. Ann Intern Med 2007;147: 357-369

152. Bax JJ, Young LH, Frye RL, Bonow RO, Steinberg HO, Barrett EJ, ADA. Screening for coronary artery disease in patients with diabetes. Diabetes Care 2007; 30:2729-2736

153. Berger M, Berchtold P, Cüppers HJ, Drost H, Kley HK, Müller WA, Wiegelmann W, Zimmerman-Telschow H, Gries FA, Krüskemper HL, Zimmermann H. Metabolic and hormonal effects of muscular exercise in juvenile type diabetics. Diabetologia 1977;13: 355-365

154. American Diabetes Association: Physical activity/exercise and diabetes (Position Statement). Diabetes Care 2004; 27(Suppl. 1):S58-S62

155. Berger M: Adjustment of insulin and oral agent therapy. In Handbook of Exercise in Diabetes. 2nd ed. Ruderman N, Devlin JTSSH, Krisska A, Eds. Alexandria, VA, American Diabetes Association, 2002, p. 365-376
156. Aiello LP, Wong J, Cavallerano J, Bursell SE, Aiello LM: Retinopathy. In Handbook of Exercise in Diabetes. 2nd ed. Ruderman N, Devlin JT, Kriska A, Eds. Alexandria, VA, American Diabetes Association, 2002, p. 401-413

157. Lemaster JW, Reiber GE, Smith DG, Heagerty PJ, Wallace C. Daily weightbearing activity does not increase the risk of diabetic foot ulcers. Med Sci Sports Exerc 2003;35:1093-1099

158. Vinik A, Erbas T: Neuropathy. In Handbook of Exercise in Diabetes. 2nd ed. Ruderman N, Devlin JT, Kriska A, Eds. Alexandria, VA, American Diabetes Association, 2002, p. 463-496

159. Wackers FJ, Young LH, Inzucchi SE, Chyun DA, Davey JA, Barrett EJ, Taillefer R, Wittlin SD, Heller GV, Filipchuk N, Engel S, Ratner RE, Iskandrian $\mathrm{AE}$, Detection of Ischemia in Asymptomatic Diabetics Investigators. Detection of silent myocardial ischemia in asymptomatic diabetic subjects: the DIAD study. Diabetes Care 2004;27: 1954-1961

160. Valensi P, Sachs RN, Harfouche B, Lormeau B, Paries J, Cosson E, Paycha F, Leutenegger M, Attali JR. Predictive value of cardiac autonomic neuropathy in diabetic patients with or without silent myocardial ischemia. Diabetes Care 2001;24:339-343

161. Mogensen CE: Nephropathy. In Handbook of Exercise in Diabetes. 2nd ed. Ruderman N, Devlin JT, Kriska A, Eds. Alexandria, VA, American Diabetes Association, 2002, p. 433-449

162. Anderson RJ, Grigsby AB, Freedland KE, de Groot M, McGill JB, Clouse RE, Lustman PJ. Anxiety and poor glycemic control: a meta-analytic review of the literature. Int J Psychiatry Med 2002;32: 235-247

163. Delahanty LM, Grant RW, Wittenberg E, Bosch JL, Wexler DJ, Cagliero E, Meigs JB. Association of diabetes-related emotional distress with diabetes treatment in primary care patients with Type 2 diabetes. Diabet Med 2007;24:48-54

164. American Diabetes Association: Psychosocial factors affecting adherence, quality of life, and well-being: Helping Patients cope. In Medical Management of Type 1 Diabetes. 5 ed. Francine R. Kaufman, Ed. Alexandria, VA, American Diabetes Association, 2008, p. 173-193

165. Fisher L, Skaff MM, Mullan JT, Arean P, Mohr D, Masharani U, Glasgow R, Laurencin $\mathrm{G}$. Clinical depression versus distress among patients with type 2 diabetes: not just a question of semantics. Diabetes Care 2007;30:542-548

166. Surwit RS, Schneider MS, Feinglos MN. Stress and diabetes mellitus. Diabetes Care 1992; 15:1413-1422

167. McCulloch DK, Glasgow RE, Hampson SE, Wagner E. A systematic approach to 
diabetes management in the post-DCCT era. Diabetes Care 1994;17:765-769

168. Rubin RR, Peyrot M. Psychological issues and treatments for people with diabetes. J Clin Psychol 2001;57:457-478

169. Jacobson AM. Depression and diabetes. Diabetes Care 1993;16:1621-1623

170. Lustman PJ, Griffith LS, Clouse RE, Cryer PE. Psychiatric illness in diabetes mellitus. Relationship to symptoms and glucose control. J Nerv Ment Dis 1986; 174:736-742

171. Blonde L, Merilainen M, Karwe V, Raskin P, TITRATE Study Group. Patient-directed titration for achieving glycaemic goals using a once-daily basal insulin analogue: an assessment of two different fasting plasma glucose targets -the TITRATE study. Diabetes Obes Metab 2009;11:623-631

172. American Diabetes Association: Hyperglycemic crises in diabetes. Diabetes Care 2004;27 (Suppl. 1):S94-S102

173. Kitabchi AE, Umpierrez GE, Miles JM, Fisher JN. Hyperglycemic crises in adult patients with diabetes. Diabetes Care 2009;32:1335-1343

174. Cryer PE. Hypoglycaemia: the limiting factor in the glycaemic management of Type I and Type II diabetes. Diabetologia 2002; 45:937-948

175. Gannon MC, Nuttall FQ: Protein and Diabetes. In American Diabetes Association Guide to Medical Nutrition Therapy for Diabetes. Franz MJ, Bantle JP, Eds. Alexandria, VA, American Diabetes Association, 1999, p. 107-125

176. Cryer PE. Diverse causes of hypoglycemia-associated autonomic failure in diabetes. N Engl J Med 2004;350:22722279

177. Cryer PE, Davis SN, Shamoon H. Hypoglycemia in diabetes. Diabetes Care 2003;26:1902-1912

178. Smith SA, Poland GA. Use of influenza and pneumococcal vaccines in people with diabetes. Diabetes Care 2000;23: 95-108

179. Colquhoun AJ, Nicholson KG, Botha JL, Raymond NT. Effectiveness of influenza vaccine in reducing hospital admissions in people with diabetes. Epidemiol Infect 1997;119:335-341

180. Bridges CB, Fukuda K, Uyeki TM, Cox NJ, Singleton JA. Prevention and control of influenza. Recommendations of the Advisory Committee on Immunization Practices (ACIP). MMWR Recomm Rep 2002;51:1-31

181. American Diabetes Association: Influenza and pneumococcal immunization in diabetes (Position Statement). Diabetes Care 2004;27(Suppl. 1):S111-S113

182. Gaede P, Lund-Andersen H, Parving $\mathrm{HH}$, Pedersen $\mathrm{O}$. Effect of a multifactorial intervention on mortality in type 2 diabetes. N Engl J Med 2008;358:580591
183. Buse JB, Ginsberg HN, Bakris GL, Clark NG, Costa F, Eckel R, Fonseca V, Gerstein HC, Grundy S, Nesto RW, Pignone MP, Plutzky J, Porte D, Redberg R, Stitzel KF, Stone NJ, American Heart Association, American Diabetes Association. Primary prevention of cardiovascular diseases in people with diabetes mellitus: a scientific statement from the American Heart Association and the American Diabetes Association. Diabetes Care 2007;30:162-172

184. Chobanian AV, Bakris GL, Black HR, Cushman WC, Green LA, Izzo JL Jr, Jones DW, Materson BJ, Oparil S, Wright JT Jr, Roccella EJ, National Heart, Lung, and Blood Institute Joint National Committee on Prevention, Detection, Evaluation, and Treatment of High Blood Pressure, National High Blood Pressure Education Program Coordinating Committee. The Seventh Report of the Joint National Committee on Prevention, Detection, Evaluation, and Treatment of High Blood Pressure: the JNC 7 report. JAMA 2003;289:2560-2572

185. Bobrie G, Chatellier G, Genes N, Clerson P, Vaur L, Vaisse B, Menard J, Mallion JM. Cardiovascular prognosis of "masked hypertension" detected by blood pressure self-measurement in elderly treated hypertensive patients. JAMA 2004;291:1342-1349

186. Sega R, Facchetti R, Bombelli M, Cesana G, Corrao G, Grassi G, Mancia G. Prognostic value of ambulatory and home blood pressures compared with office blood pressure in the general population: follow-up results from the Pressioni Arteriose Monitorate e Loro Associazioni (PAMELA) study. Circulation 2005;111:1777-1783

187. UKPDS: Tight blood pressure control and risk of macrovascular and microvascular complications in type 2 diabetes: UKPDS 38. UK Prospective Diabetes Study Group. BMJ 1998;317:703-713

188. Hansson L, Zanchetti A, Carruthers SG, Dahlöf B, Elmfeldt D, Julius S, Ménard J, Rahn KH, Wedel H, Westerling S. Effects of intensive blood-pressure lowering and low-dose aspirin in patients with hypertension: principal results of the Hypertension Optimal Treatment (HOT) randomised trial. HOT Study Group. Lancet 1998;351:1755-1762

189. Adler AI, Stratton IM, Neil HA, Yudkin JS, Matthews DR, Cull CA, Wright AD, Turner RC, Holman RR. Association of systolic blood pressure with macrovascular and microvascular complications of type 2 diabetes (UKPDS 36): prospective observational study. BMJ 2000;321: 412-419

190. Lewington S, Clarke R, Qizilbash N, Peto R, Collins R, Prospective Studies Collaboration. Age-specific relevance of usual blood pressure to vascular mortal- ity: a meta-analysis of individual data for one million adults in 61 prospective studies. Lancet 2002;360:1903-1913

191. Stamler J, Vaccaro O, Neaton JD, Wentworth D. Diabetes, other risk factors, and 12-yr cardiovascular mortality for men screened in the Multiple Risk Factor Intervention Trial. Diabetes Care 1993; 16:434-444

192. Cushman WC, Grimm RH Jr, Cutler JA, Evans GW, Capes S, Corson MA, Sadler LS, Alderman MH, Peterson K, Bertoni A, Basile JN, ACCORD Study Group: Rationale and design for the blood pressure intervention of the Action to Control Cardiovascular Risk in Diabetes (ACCORD) trial. Am J Cardiol 2007;99:44i$55 \mathrm{i}$

193. Sacks FM, Svetkey LP, Vollmer WM, Appel LJ, Bray GA, Harsha D, Obarzanek E, Conlin PR, Miller ER 3rd, Simons-Morton DG, Karanja N, Lin PH, DASH-Sodium Collaborative Research Group. Effects on blood pressure of reduced dietary sodium and the Dietary Approaches to Stop Hypertension (DASH) diet. DASH-Sodium Collaborative Research Group. N Engl J Med 2001;344: 3-10

194. Tatti P, Pahor M, Byington RP, Di Mauro P, Guarisco R, Strollo G, Strollo F. Outcome results of the Fosinopril Versus Amlodipine Cardiovascular Events Randomized Trial (FACET) in patients with hypertension and NIDDM. Diabetes Care 1998;21:597-603

195. Estacio RO, Jeffers BW, Hiatt WR, Biggerstaff SL, Gifford N, Schrier RW. The effect of nisoldipine as compared with enalapril on cardiovascular outcomes in patients with non-insulin-dependent diabetes and hypertension. N Engl J Med 1998;338:645-652

196. Schrier RW, Estacio RO, Mehler PS, Hiatt WR. Appropriate blood pressure control in hypertensive and normotensive type 2 diabetes mellitus: a summary of the ABCD trial. Nat Clin Pract Nephrol 2007;3:428-438

197. ALLHAT Officers and Coordinators for the ALLHAT Collaborative Research Group. The Antihypertensive and LipidLowering Treatment to Prevent Heart Attack Trial: the Antihypertensive and Lipid-Lowering Treatment to Prevent Heart Attack Trial (ALLHAT). JAMA 2002;288:2981-2997

198. Psaty BM, Lumley T, Furberg CD, Schellenbaum G, Pahor M, Alderman MH, Weiss NS. Health outcomes associated with various antihypertensive therapies used as first-line agents: a network metaanalysis. JAMA 2003;289:2534-2544

199. Effects of ramipril on cardiovascular and microvascular outcomes in people with diabetes mellitus: results of the HOPE study and MICRO-HOPE substudy: Heart Outcomes Prevention Evaluation 
Study Investigators. Lancet 2000;355: 253-259

200. Pfeffer MA, Swedberg K, Granger CB, Held P, McMurray JJ, Michelson EL, Olofsson B, Ostergren J, Yusuf S, Pocock $S$, CHARM Investigators and Committees. Effects of candesartan on mortality and morbidity in patients with chronic heart failure: the CHARM-Overall programme. Lancet 2003;362:759-766

201. Granger CB, McMurray JJ, Yusuf S, Held $\mathrm{P}$, Michelson EL, Olofsson B, Ostergren J, Pfeffer MA, Swedberg K, CHARM Investigators and Committees. Effects of candesartan in patients with chronic heart failure and reduced left-ventricular systolic function intolerant to angiotensin-converting-enzyme inhibitors: the CHARM-Alternative trial. Lancet 2003; 362:772-776

202. McMurray JJ, Ostergren J, Swedberg K, Granger CB, Held P, Michelson EL, Olofsson B, Yusuf S, Pfeffer MA, CHARM Investigators and Committees. Effects of candesartan in patients with chronic heart failure and reduced leftventricular systolic function taking angiotensin-converting-enzyme

inhibitors: the CHARM-Added trial. Lancet 2003;362:767-771

203. Lindholm LH, Ibsen H, Dahlöf B, Devereux RB, Beevers G, de Faire U, Fyhrquist $\mathrm{F}$, Julius $\mathrm{S}$, Kjeldsen SE, Kristiansson K, Lederballe-Pedersen $\mathrm{O}$, Nieminen MS, Omvik P, Oparil S, Wedel H, Aurup P, Edelman J, Snapinn S, LIFE Study Group. Cardiovascular morbidity and mortality in patients with diabetes in the Losartan Intervention For Endpoint reduction in hypertension study (LIFE): a randomised trial against atenolol. Lancet 2002;359:1004-1010

204. Berl T, Hunsicker LG, Lewis JB, Pfeffer MA, Porush JG, Rouleau JL, Drury PL, Esmatjes E, Hricik D, Parikh CR, Raz I, Vanhille P, Wiegmann TB, Wolfe BM, Locatelli F, Goldhaber SZ, Lewis EJ, Irbesartan Diabetic Nephropathy Trial. Collaborative Study Group. Collaborative Study Group. Cardiovascular outcomes in the Irbesartan Diabetic Nephropathy Trial of patients with type 2 diabetes and overt nephropathy. Ann Intern Med 2003;138:542-549

205. Laffel LM, McGill JB, Gans DJ. The beneficial effect of angiotensin-converting enzyme inhibition with captopril on diabetic nephropathy in normotensive IDDM patients with microalbuminuria. North American Microalbuminuria Study Group. Am J Med 1995;99:497504

206. Bakris GL, Williams M, Dworkin L, Elliott WJ, Epstein M, Toto R, Tuttle K, Douglas J, Hsueh W, Sowers J. Preserving renal function in adults with hypertension and diabetes: a consensus approach: National Kidney Foundation
Hypertension and Diabetes Executive Committees Working Group. Am J Kidney Dis 2000;36:646-661

207. Psaty BM, Smith NL, Siscovick DS, Koepsell TD, Weiss NS, Heckbert SR, Lemaitre RN, Wagner EH, Furberg CD. Health outcomes associated with antihypertensive therapies used as first-line agents: a systematic review and metaanalysis. JAMA 1997;277:739-745

208. Patel A, ADVANCE Collaborative Group, MacMahon S, Chalmers J, Neal B, Woodward M, Billot L, Harrap S, Poulter N, Marre M, Cooper M, Glasziou P, Grobbee DE, Hamet P, Heller S, Liu LS, Mancia G, Mogensen CE, Pan CY, Rodgers A, Williams B. Effects of a fixed combination of perindopril and indapamide on macrovascular and microvascular outcomes in patients with type 2 diabetes mellitus (the ADVANCE trial): a randomised controlled trial. Lancet 2007;370:829-840

209. Sibai BM. Treatment of hypertension in pregnant women. N Engl J Med 1996; 335:257-265

210. Baigent C, Keech A, Kearney PM, Blackwell L, Buck G, Pollicino C, Kirby A, Sourjina T, Peto R, Collins R, Simes R, Cholesterol Treatment Trialists' (CTT) Collaborators. Efficacy and safety of cholesterol-lowering treatment: prospective meta-analysis of data from 90,056 participants in 14 randomised trials of statins. Lancet 2005;366:12671278

211. Pyŏrälä K, Pedersen TR, Kjekshus J, Faergeman O, Olsson AG, Thorgeirsson G. Cholesterol lowering with simvastatin improves prognosis of diabetic patients with coronary heart disease: a subgroup analysis of the Scandinavian Simvastatin Survival Study (4S). Diabetes Care 1997;20:614-620

212. Collins R, Armitage J, Parish S, Sleigh P, Peto R, Heart Protection Study Collaborative Group: MRC/BHF Heart Protection Study of cholesterol-lowering with simvastatin in 5963 people with diabetes: a randomised placebo-controlled trial. Lancet 2003;361:2005-2016

213. Goldberg RB, Mellies MJ, Sacks FM, Moyé LA, Howard BV, Howard WJ, Davis BR, Cole TG, Pfeffer MA, Braunwald E. Cardiovascular events and their reduction with pravastatin in diabetic and glucose-intolerant myocardial infarction survivors with average cholesterol levels: subgroup analyses in the cholesterol and recurrent events (CARE) trial. The Care Investigators. Circulation 1998;98:2513-2519

214. Shepherd J, Barter P, Carmena R, Deedwania P, Fruchart JC, Haffner S, Hsia J, Breazna A, LaRosa J, Grundy S, Waters D. Effect of lowering LDL cholesterol substantially below currently recommended levels in patients with coronary heart disease and diabetes: the Treating to New Targets (TNT) study. Diabetes Care 2006;29:1220-1226

215. Sever PS, Poulter NR, Dahlöf B, Wedel H, Collins R, Beevers G, Caulfield M, Kjeldsen SE, Kristinsson A, McInnes GT, Mehlsen J, Nieminen M, O’Brien E, Ostergren J. Reduction in cardiovascular events with atorvastatin in 2,532 patients with type 2 diabetes: Anglo-Scandinavian Cardiac Outcomes Trial-lipidlowering arm (ASCOT-LLA). Diabetes Care 2005;28:1151-1157

216. Knopp RH, d'Emden M, Smilde JG, Pocock SJ. Efficacy and safety of atorvastatin in the prevention of cardiovascular end points in subjects with type 2 diabetes: the Atorvastatin Study for Prevention of Coronary Heart Disease Endpoints in non-insulin-dependent diabetes mellitus (ASPEN). Diabetes Care 2006;29:1478-1485

217. Singh IM, Shishehbor MH, Ansell BJ. High-density lipoprotein as a therapeutic target: a systematic review. JAMA 2007;298:786-798

218. Canner PL, Berge KG, Wenger NK, Stamler J, Friedman L, Prineas RJ, Friedewald W. Fifteen year mortality in Coronary Drug Project patients: longterm benefit with niacin. J Am Coll Cardiol 1986;8:1245-1255

219. Rubins HB, Robins SJ, Collins D, Fye CL, Anderson JW, Elam MB, Faas FH, Linares E, Schaefer EJ, Schectman G, Wilt TJ, Wittes J. Gemfibrozil for the secondary prevention of coronary heart disease in men with low levels of high-density lipoprotein cholesterol. Veterans Affairs High-Density Lipoprotein Cholesterol Intervention Trial Study Group. N Engl J Med 1999;341:410-418

220. Frick MH, Elo O, Haapa K, Heinonen OP, Heinsalmi P, Helo P, Huttunen JK, Kaitaniemi P, Koskinen P, Manninen V: Helsinki Heart Study: primary-prevention trial with gemfibrozil in middleaged men with dyslipidemia: safety of treatment, changes in risk factors, and incidence of coronary heart disease. N Engl J Med 1987;317:1237-1245

221. Keech A, Simes RJ, Barter P, Best J, Scott R, Taskinen MR, Forder P, Pillai A, Davis T, Glasziou P, Drury P, Kesäniemi YA, Sullivan D, Hunt D, Colman P, d'Emden M, Whiting M, Ehnholm C, Laakso M, FIELD study investigators. Effects of long-term fenofibrate therapy on cardiovascular events in 9795 people with type 2 diabetes mellitus (the FIELD study): randomised controlled trial. Lancet 2005;366:1849-1861

222. Expert Panel on Detection, Evaluation, and Treatment of High Blood Cholesterol in Adults: Executive Summary of The Third Report of The National Cholesterol Education Program (NCEP) Expert Panel on Detection, Evaluation, 
And Treatment of High Blood Cholesterol In Adults (Adult Treatment Panel III). JAMA 2001;285:2486-2497

223. Hayward RA, Hofer TP, Vijan S. Narrative review: lack of evidence for recommended low-density lipoprotein treatment targets: a solvable problem. Ann Intern Med 2006;145:520-530

224. Cannon CP, Braunwald E, McCabe CH, Rader DJ, Rouleau JL, Belder R, Joyal SV, Hill KA, Pfeffer MA, Skene AM, Pravastatin or Atorvastatin Evaluation and Infection Therapy-Thrombolysis in Myocardial Infarction 22 Investigators. Intensive versus moderate lipid lowering with statins after acute coronary syndromes. N Engl J Med 2004;350:14951504

225. de Lemos JA, Blazing MA, Wiviott SD, Lewis EF, Fox KA, White HD, Rouleau JL, Pedersen TR, Gardner LH, Mukherjee R, Ramsey KE, Palmisano J, Bilheimer DW, Pfeffer MA, Califf RM, Braunwald E, A to Z Investigators. Early intensive vs a delayed conservative simvastatin strategy in patients with acute coronary syndromes: phase $\mathrm{Z}$ of the A to Z trial. JAMA 2004;292:1307-1316

226. Nissen SE, Tuzcu EM, Schoenhagen P, Brown BG, Ganz P, Vogel RA, Crowe T, Howard G, Cooper CJ, Brodie B, Grines CL, DeMaria AN, REVERSAL Investigators. Effect of intensive compared with moderate lipid-lowering therapy on progression of coronary atherosclerosis: a randomized controlled trial. JAMA 2004;291:1071-1080

227. Grundy SM, Cleeman JI, Merz CN, Brewer HB Jr, Clark LT, Hunninghake DB, Pasternak RC, Smith SC Jr, Stone NJ, National Heart, Lung, and Blood Institute, American College of Cardiology Foundation, American Heart Association. Implications of recent clinical trials for the National Cholesterol Education Program Adult Treatment Panel III guidelines. Circulation 2004;110:227-239

228. Chasman DI, Posada D, Subrahmanyan L, Cook NR, Stanton VP, Jr, Ridker PM. Pharmacogenetic study of statin therapy and cholesterol reduction. JAMA 2004; 291:2821-2827

229. Baigent C, Keech A, Kearney PM, Blackwell L, Buck G, Pollicino C, Kirby A, Sourjina T, Peto R, Collins R, Simes R, Cholesterol Treatment Trialists' (CTT) Collaborators. Efficacy and safety of cholesterol-lowering treatment: prospective meta-analysis of data from 90,056 participants in 14 randomised trials of statins. Lancet 2005;366:12671278

230. Elam MB, Hunninghake DB, Davis KB, Garg R, Johnson C, Egan D, Kostis JB, Sheps DS, Brinton EA. Effect of niacin on lipid and lipoprotein levels and glycemic control in patients with diabetes and peripheral arterial disease: the ADMIT study: A randomized trial. Arterial Disease Multiple Intervention Trial. JAMA 2000;284:1263-1270

231. Grundy SM, Vega GL, McGovern ME, Tulloch BR, Kendall DM, Fitz-Patrick D, Ganda OP, Rosenson RS, Buse JB, Robertson DD, Sheehan JP, Diabetes Multicenter Research Group. Efficacy, safety, and tolerability of once-daily niacin for the treatment of dyslipidemia associated with type 2 diabetes: results of the assessment of diabetes control and evaluation of the efficacy of niaspan trial. Arch Intern Med 2002;162:1568-1576

232. Jones $\mathrm{PH}$, Davidson $\mathrm{MH}$. Reporting rate of rhabdomyolysis with fenofibrate + statin versus gemfibrozil + any statin. Am J Cardiol 2005;95:120-122

233. Brunzell JD, Davidson M, Furberg CD Goldberg RB, Howard BV, Stein JH, Witztum JL, American Diabetes Association, American College of Cardiology Foundation. Lipoprotein management in patients with cardiometabolic risk: consensus statement from the American Diabetes Association and the American College of Cardiology Foundation. Diabetes Care 2008;31:811-822

234. Colhoun HM, Betteridge DJ, Durrington PN, Hitman GA, Neil HA, Livingstone SJ, Thomason MJ, Mackness MI, CharltonMenys V, Fuller JH, CARDS investigators. Primary prevention of cardiovascular disease with atorvastatin in type 2 diabetes in the Collaborative Atorvastatin Diabetes Study (CARDS): multicentre randomised placebo-controlled trial. Lancet 2004;364: 685-696

235. Buse JB, Ginsberg HN, Bakris GL, Clark NG, Costa F, Eckel R, Fonseca V, Gerstein HC, Grundy S, Nesto RW, Pignone MP, Plutzky J, Porte D, Redberg R, Stitzel KF, Stone NJ, American Heart Association, American Diabetes Association. Primary prevention of cardiovascular diseases in people with diabetes mellitus: a scientific statement from the American Heart Association and the American Diabetes Association. Diabetes Care 2007:30:162-172

236. Antithrombotic Trialists' (ATT) Collaboration, Baigent C, Blackwell L, Collins R, Emberson J, Godwin J, Peto R, Buring J, Hennekens C, Kearney P, Meade T, Patrono C, Roncaglioni MC, Zanchetti A. Aspirin in the primary and secondary prevention of vascular disease: collaborative meta-analysis of individual participant data from randomised trials. Lancet 2009;373:1849-1860

237. Wolff T, Miller T, Ko S. Aspirin for the primary prevention of cardiovascular events: an update of the evidence for the U.S. Preventive Services Task Force. Ann Intern Med 2009;150:405-410

238. US Preventive Services Task Force: Aspirin for the prevention of cardiovascular disease: U.S. Preventive Services Task
Force recommendation statement. Ann Intern Med 2009;150:396-404

239. Ogawa H, Nakayama M, Morimoto T, Uemura S, Kanauchi M, Doi N, Jinnouchi H, Sugiyama S, Saito Y, Japanese Primary Prevention of Atherosclerosis With Aspirin for Diabetes (JPAD) Trial Investigators. Low-dose aspirin for primary prevention of atherosclerotic events in patients with type 2 diabetes: a randomized controlled trial. JAMA 2008;300: 2134-2141

240. Belch J, MacCuish A, Campbell I, Cobbe S, Taylor R, Prescott R, Lee R, Bancroft J, MacEwan S, Shepherd J, Macfarlane P, Morris A, Jung R, Kelly C, Connacher A, Peden N, Jamieson A, Matthews D, Leese G, McKnight J, O’Brien I, Semple C, Petrie J, Gordon D, Pringle S, MacWalter R. The prevention of progression of arterial disease and diabetes (POPADAD) trial: factorial randomised placebo controlled trial of aspirin and antioxidants in patients with diabetes and asymptomatic peripheral arterial disease. BMJ 2008; 337:a1840

241. Pignone M, Earnshaw S, Tice JA, Pletcher MJ. Aspirin, statins, or both drugs for the primary prevention of coronary heart disease events in men: a cost-utility analysis. Ann Intern Med 2006; 144:326-336

242. Campbell CL, Smyth S, Montalescot G, Steinhubl SR. Aspirin dose for the prevention of cardiovascular disease: a systematic review. JAMA 2007;297:20182024

243. Davì G, Patrono C. Platelet activation and atherothrombosis. N Engl J Med 2007;357:2482-2494

244. Watala C, Golanski J, Pluta J, Boncler M, Rozalski M, Luzak B, Kropiwnicka A, Drzewoski J. Reduced sensitivity of platelets from type 2 diabetic patients to acetylsalicylic acid (aspirin)-its relation to metabolic control. Thromb Res 2004; 113:101-113

245. Lev EI. Aspirin resistance transient laboratory finding or important clinical entity? J Am Coll Cardiol 2009;53:678680

246. Santilli F, Rocca B, De Cristofaro R, Lattanzio S, Pietrangelo L, Habib A, Pettinella C, Recchiuti A, Ferrante E, Ciabattoni G, Davì G, Patrono C. Platelet cyclooxygenase inhibition by low-dose aspirin is not reflected consistently by platelet function assays: implications for aspirin "resistance". J Am Coll Cardiol 2009;53:667-677

247. Bhatt DL, Marso SP, Hirsch AT, Ringleb PA, Hacke W, Topol EJ. Amplified benefit of clopidogrel versus aspirin in patients with diabetes mellitus. Am J Cardiol 2002;90:625-628

248. Haire-Joshu D, Glasgow RE, Tibbs TL. Smoking and diabetes. Diabetes Care 1999;22:1887-1898 
249. American Diabetes Asociation: Smoking and diabetes (Position Statement). Diabetes Care 2004;27(Suppl. 1):S74-S75

250. US Preventive Services Task Force. Counseling to Prevent Tobacco Use and Tobacco-Related Diseases: Recommendation Statement. Rockville, MD, Agency for Healthcare Research and Quality, 2003

251. Ranney L, Melvin C, Lux L, McClain E, Lohr KN. Systematic review: smoking cessation intervention strategies for adults and adults in special populations. Ann Intern Med 2006;145:845-856

252. Scognamiglio R, Negut C, Ramondo A, Tiengo A, Avogaro A. Detection of coronary artery disease in asymptomatic patients with type 2 diabetes mellitus. J Am Coll Cardiol 2006;47:65-71

253. Boden WE, O'Rourke RA, Teo KK, Hartigan PM, Maron DJ, Kostuk WJ, Knudtson M, Dada M, Casperson P, Harris CL, Chaitman BR, Shaw L, Gosselin G, Nawaz S, Title LM, Gau G, Blaustein AS, Booth DC, Bates ER, Spertus JA, Berman DS, Mancini GB, Weintraub WS, COURAGE Trial Research Group. Optimal medical therapy with or without PCI for stable coronary disease. N Engl J Med 2007;356:1503-1516

254. BARI 2D Study Group, Frye RL, August P, Brooks MM, Hardison RM, Kelsey SF, MacGregor JM, Orchard TJ, Chaitman BR, Genuth SM, Goldberg SH, Hlatky MA, Jones TL, Molitch ME, Nesto RW, Sako EY, Sobel BE. A randomized trial of therapies for type 2 diabetes and coronary artery disease. N Engl J Med 2009; 360:2503-2515

255. Wackers FJ, Chyun DA, Young LH, Heller GV, Iskandrian AE, Davey JA, Barrett EJ, Taillefer R, Wittlin SD, Filipchuk N, Ratner RE, Inzucchi SE: Resolution of asymptomatic myocardial ischemia in patients with type 2 diabetes mellitus in the DIAD Study. Diabetes Care 2007:30:2892-2898

256. Young LH, Wackers FJ, Chyun DA, Davey JA, Barrett EJ, Taillefer R, Heller GV, Iskandrian AE, Wittlin SD, Filipchuk N, Ratner RE, Inzucchi SE, DIAD Investigators. Cardiac outcomes after screening for asymptomatic coronary artery disease in patients with type 2 diabetes: the DIAD study: a randomized controlled trial. JAMA 2009;301:15471555

257. Braunwald E, Domanski MJ, Fowler SE, Geller NL, Gersh BJ, Hsia J, Pfeffer MA, Rice MM, Rosenberg YD, Rouleau JL, PEACE Trial Investigators. Angiotensinconverting-enzyme inhibition in stable coronary artery disease. N Engl J Med 2004:351:2058-2068

258. Effects of the angiotensin-receptor blocker telmisartan on cardiovascular events in high-risk patients intolerant to angiotensin-converting enzyme inhibitors: a randomised controlled trial. Lan- cet 2008;372:1174-1183

259. Garg JP, Bakris GL. Microalbuminuria: marker of vascular dysfunction, risk factor for cardiovascular disease. Vasc Med 2002; 7:35-43

260. Klausen K, Borch-Johnsen K, Feldt-Rasmussen B, Jensen G, Clausen P, Scharling H, Appleyard M, Jensen JS. Very low levels of microalbuminuria are associated with increased risk of coronary heart disease and death independently of renal function, hypertension, and diabetes. Circulation 2004;110: 32-35

261. Gall MA, Hougaard P, Borch-Johnsen K, Parving HH. Risk factors for development of incipient and overt diabetic nephropathy in patients with non-insulin dependent diabetes mellitus: prospective, observational study. BMJ 1997;314: 783-788

262. Ravid M, Lang R, Rachmani R, Lishner M. Long-term renoprotective effect of angiotensin-converting enzyme inhibition in non-insulin-dependent diabetes mellitus. A 7-year follow-up study. Arch Intern Med 1996;156:286-289

263. Reichard P, Nilsson BY, Rosenqvist U. The effect of long-term intensified insulin treatment on the development of microvascular complications of diabetes mellitus. N Engl J Med 1993;329:304-309

264. Effect of intensive therapy on the development and progression of diabetic nephropathy in the Diabetes Control and Complications Trial. The Diabetes Control and Complications (DCCT) Research Group. Kidney Int 1995;47: 1703-1720

265. Lewis EJ, Hunsicker LG, Bain RP, Rohde RD. The effect of angiotensin-converting-enzyme inhibition on diabetic nephropathy. The Collaborative Study Group. N Engl J Med 1993;329:14561462

266. Remuzzi G, Macia M, Ruggenenti P. Prevention and treatment of diabetic renal disease in type 2 diabetes: the BENEDICT study. JAmSocNephrol 2006;17: S90-S97

267. Bilous R, Chaturvedi N, Sjølie AK, Fuller J, Klein R, Orchard T, Porta M, Parving HH. Effect of candesartan on microalbuminuria and albumin excretion rate in diabetes: three randomized trials. Ann Intern Med 2009;151:11-14

268. Mauer M, Zinman B, Gardiner R, Suissa S, Sinaiko A, Strand T, Drummond K, Donnelly S, Goodyer P, Gubler MC, Klein R. Renal and retinal effects of enalapril and losartan in type 1 diabetes. N Engl J Med 2009;361:40-51

269. Lewis EJ, Hunsicker LG, Clarke WR, Berl T, Pohl MA, Lewis JB, Ritz E, Atkins RC, Rohde R, Raz I, Collaborative Study Group. Renoprotective effect of the angiotensin-receptor antagonist irbesartan in patients with nephropathy due to type
2 diabetes. N Engl J Med 2001;345:851860

270. Brenner BM, Cooper ME, de Zeeuw D, Keane WF, Mitch WE, Parving HH, Remuzzi G, Snapinn SM, Zhang Z, Shahinfar S, RENAAL Study Investigators. Effects of losartan on renal and cardiovascular outcomes in patients with type 2 diabetes and nephropathy. N Engl J Med 2001;345:861-869

271. Parving HH, Lehnert H, BröchnerMortensen J, Gomis R, Andersen S, Arner P, Irbesartan in Patients with Type 2 Diabetes and Microalbuminuria Study Group. The effect of irbesartan on the development of diabetic nephropathy in patients with type 2 diabetes. N Engl J Med 2001;345:870-878

272. Pepine CJ, Handberg EM, Cooper-DeHoff RM, Marks RG, Kowey P, Messerli FH, Mancia G, Cangiano JL, Garcia-Barreto D, Keltai M, Erdine S, Bristol HA, Kolb HR, Bakris GL, Cohen JD, Parmley WW, INVEST Investigators. A calcium antagonist vs a non-calcium antagonist hypertension treatment strategy for patients with coronary artery disease: the International Verapamil-Trandolapril study (INVEST): a randomized controlled trial. JAMA 2003;290:28052816

273. Bakris GL, Siomos M, Richardson D, Janssen I, Bolton WK, Hebert L, Agarwal R, Catanzaro D. ACE inhibition or angiotensin receptor blockade: impact on potassium in renal failure. VAL-K Study Group. Kidney Int 2000;58:2084-2092

274. Mogensen CE, Neldam S, Tikkanen I, Oren S, Viskoper R, Watts RW, Cooper ME. Randomised controlled trial of dual blockade of renin-angiotensin system in patients with hypertension, microalbuminuria, and non-insulin dependent diabetes: the candesartan and lisinopril microalbuminuria (CALM) study. BMJ 2000;321:1440-1444

275. Schjoedt KJ, Jacobsen P, Rossing K, Boomsma F, Parving HH. Dual blockade of the renin-angiotensin-aldosterone system in diabetic nephropathy: the role of aldosterone. Horm Metab Res 2005; 37(Suppl. 1):4-8

276. Schjoedt KJ, Rossing K, Juhl TR, Boomsma F, Rossing P, Tarnow L, Parving $\mathrm{HH}$. Beneficial impact of spironolactone in diabetic nephropathy. Kidney Int 2005:68:2829-2836

277. Parving HH, Persson F, Lewis JB, Lewis EJ, Hollenberg NK, AVOID Study Investigators. Aliskiren combined with losartan in type 2 diabetes and nephropathy. N Engl J Med 2008;358:2433-2446

278. Pijls LT, de Vries H, Donker AJ, van Eijk JT. The effect of protein restriction on albuminuria in patients with type 2 diabetes mellitus: a randomized trial. Nephrol Dial Transplant 1999; 14:14451453 
279. Pedrini MT, Levey AS, Lau J, Chalmers TC, Wang PH. The effect of dietary protein restriction on the progression of diabetic and nondiabetic renal diseases: a meta-analysis. Ann Intern Med 1996; 124:627-632

280. Hansen HP, Tauber-Lassen E, Jensen BR, Parving HH. Effect of dietary protein restriction on prognosis in patients with diabetic nephropathy. Kidney Int 2002; 62:220-228

281. Kasiske BL, Lakatua JD, Ma JZ, Louis TA. A meta-analysis of the effects of dietary protein restriction on the rate of decline in renal function. Am J Kidney Dis 1998; 31:954-961

282. Eknoyan G, Hostetter T, Bakris GL, Hebert L, Levey AS, Parving HH, Steffes MW, Toto R. Proteinuria and other markers of chronic kidney disease: a position statement of the national kidney foundation (NKF) and the national institute of diabetes and digestive and kidney diseases (NIDDK). Am J Kidney Dis 2003;42:617-622

283. Levey AS, Coresh J, Balk E, Kausz AT, Levin A, Steffes MW, Hogg RJ, Perrone RD, Lau J, Eknoyan G, National Kidney Foundation. National Kidney Foundation practice guidelines for chronic kidney disease: evaluation, classification, and stratification. Ann Intern Med 2003; 139:137-147

284. Kramer H, Molitch ME. Screening for kidney disease in adults with diabetes. Diabetes Care 2005;28:1813-1816

285. Kramer HJ, Nguyen QD, Curhan G, Hsu CY. Renal insufficiency in the absence of albuminuria and retinopathy among adults with type 2 diabetes mellitus. JAMA 2003;289:3273-3277

286. Tsalamandris C, Allen TJ, Gilbert RE, Sinha A, Panagiotopoulos S, Cooper ME, Jerums G. Progressive decline in renal function in diabetic patients with and without albuminuria. Diabetes 1994:43: 649-655

287. Levey AS, Bosch JP, Lewis JB, Greene T, Rogers N, Roth D. A more accurate method to estimate glomerular filtration rate from serum creatinine: a new prediction equation. Modification of Diet in Renal Disease Study Group. Ann Intern Med 1999; 130:461-470

288. Rigalleau V, Lasseur C, Perlemoine C, Barthe N, Raffaitin C, Liu C, Chauveau $\mathrm{P}$, Baillet-Blanco L, Beauvieux MC, Combe C, Gin H. Estimation of glomerular filtration rate in diabetic subjects: Cockcroft formula or modification of Diet in Renal Disease study equation? Diabetes Care 2005;28:838-843

289. Levinsky NG. Specialist evaluation in chronic kidney disease: too little, too late. Ann Intern Med 2002;137:542543

290. American Diabetes Association: Nephropathy in diabetes (Position State- ment). Diabetes Care 2004;27(Suppl. 1):S79-S83

291. Klein R. Hyperglycemia and microvascular and macrovascular disease in diabetes. Diabetes Care 1995;18:258-268

292. Estacio RO, McFarling E, Biggerstaff S, Jeffers BW, Johnson D, Schrier RW. Overt albuminuria predicts diabetic retinopathy in Hispanics with NIDDM. Am J Kidney Dis 1998;31:947-953

293. Leske MC, Wu SY, Hennis A, Hyman L, Nemesure B, Yang L, Schachat AP, Barbados Eye Study Group. Hyperglycemia, blood pressure, and the 9-year incidence of diabetic retinopathy: the Barbados Eye Studies. Ophthalmology 2005;112: 799-805

294. Fong DS, Aiello LP, Ferris FL 3rd, Klein R. Diabetic retinopathy. Diabetes Care 2004:27:2540-2553

295. Diabetes Control and Complications Trial Research Group: Effect of pregnancy on microvascular complications in the diabetes control and complications trial. The Diabetes Control and Complications Trial Research Group. Diabetes Care 2000;23:1084-1091

296. The Diabetic Retinopathy Study (DRS) Research Group. Preliminary report on the effects of photocoagulation therapy: DRS Report \#1. Am J Ophthalmol 1976; 81:383-396.

297. Photocoagulation for diabetic macular edema. Early Treatment Diabetic Retinopathy Study report number 1. Early Treatment Diabetic Retinopathy Study research group. Arch Ophthalmol 1985; 103:1796-1806

298. Klein R, Klein BE, Moss SE, Davis MD, DeMets DL. The Wisconsin epidemiologic study of diabetic retinopathy. II. Prevalence and risk of diabetic retinopathy when age at diagnosis is less than 30 years. Arch Ophthalmol 1984;102:520-526

299. Harris MI, Klein R, Welborn TA, Knuiman MW. Onset of NIDDM occurs at least 4-7 yr before clinical diagnosis. Diabetes Care 1992;15:815-819

300. Vijan S, Hofer TP, Hayward RA. Costutility analysis of screening intervals for diabetic retinopathy in patients with type 2 diabetes mellitus. JAMA 2000; 283:889-896

301. Klein R. Screening interval for retinopathy in type 2 diabetes. Lancet 2003;361: 190-191

302. Younis N, Broadbent DM, Vora JP, Harding SP, Liverpool Diabetic Eye Study. Incidence of sight-threatening retinopathy in patients with type 2 diabetes in the Liverpool Diabetic Eye Study: a cohort study. Lancet 2003;361:195-200

303. Ahmed J, Ward TP, Bursell SE, Aiello LM, Cavallerano JD, Vigersky RA. The sensitivity and specificity of nonmydriatic digital stereoscopic retinal imaging in detecting diabetic retinopathy. Diabetes Care 2006;29:2205-2209
304. American Diabetes Association: Retinopathy in diabetes. Diabetes Care 2004;27 Suppl. 1:S84-S87

305. Ciulla TA, Amador AG, Zinman B. Diabetic retinopathy and diabetic macular edema: pathophysiology, screening, and novel therapies. Diabetes Care 2003;26: 2653-2664

306. Boulton AJ, Vinik AI, Arezzo JC, Bril V, Feldman EL, Freeman R, Malik RA, Maser RE, Sosenko JM, Ziegler D, American Diabetes Association. Diabetic neuropathies: a statement by the American Diabetes Association. Diabetes Care 2005; 28:956-962

307. Vinik AI, Maser RE, Mitchell BD, Freeman R. Diabetic autonomic neuropathy. Diabetes Care 2003;26:1553-1579

308. Boulton AJ, Armstrong DG, Albert SF, Frykberg RG, Hellman R, Kirkman MS, Lavery LA, Lemaster JW, Mills JL Sr, Mueller MJ, Sheehan P, Wukich DK, American Diabetes Association, American Association of Clinical Endocrinologists. Comprehensive foot examination and risk assessment: a report of the task force of the foot care interest group of the American Diabetes Association, with endorsement by the American Association of Clinical Endocrinologists. Diabetes Care 2008;31:1679-1685

309. American Diabetes Association: Peripheral arterial disease in people with diabetes (Consensus Statement). Diabetes Care 2003;26:3333-3341

310. American Diabetes Association: Consensus Development Conference on Diabetic Foot Wound Care, 7-8 April 1999, Boston, Massachusetts. Diabetes Care 1999;22:1354-1360

311. Silverstein J, Klingensmith G, Copeland KC, Plotnick L, Kaufman F, Laffel L, Deeb LC, Grey M, Anderson BJ, Holzmeister LA, and Clark NG Care of children and adolescents with type 1 diabetes mellitus: A statement of the American Diabetes Association. Diabetes Care 2005; 28:186-212.

312. Northam EA, Anderson PJ, Werther GA, Warne GL, Adler RG, Andrewes D. Neuropsychological complications of IDDM in children 2 years after disease onset. Diabetes Care 1998;21:379-384

313. Rovet J, Alvarez M. Attentional functioning in children and adolescents with IDDM. Diabetes Care 1997;20:803-810

314. Bjørgaas M, Gimse R, Vik T, Sand T. Cognitive function in type 1 diabetic children with and without episodes of severe hypoglycaemia. Acta Paediatr 1997;86:148-153

315. Doyle EA, Weinzimer SA, Steffen AT, Ahern JA, Vincent M, Tamborlane WV. A randomized, prospective trial comparing the efficacy of continuous subcutaneous insulin infusion with multiple daily injections using insulin glargine. Diabetes Care 2004;27:1554-1558 
316. Nimri R, Weintrob $N$, Benzaquen $H$, Ofan R, Fayman G, Phillip M. Insulin pump therapy in youth with type 1 diabetes: a retrospective paired study. Pediatrics 2006;117:2126-2131

317. Krantz JS, Mack WJ, Hodis HN, Liu CR, Liu CH, Kaufman FR. Early onset of subclinical atherosclerosis in young persons with type 1 diabetes. J Pediatr 2004; 145 : 452-457

318. Järvisalo MJ, Putto-Laurila A, Jartti L, Lehtimäki T, Solakivi T, Rönnemaa T, Raitakari OT. Carotid artery intima-media thickness in children with type 1 diabetes. Diabetes 2002;51:493-498

319. Haller MJ, Samyn M, Nichols WW, Brusko T, Wasserfall C, Schwartz RF, Atkinson M, Shuster JJ, Pierce GL, Silverstein JH. Radial artery tonometry demonstrates arterial stiffness in children with type 1 diabetes. Diabetes Care 2004:27:2911-2917

320. Orchard TJ, Forrest KY, Kuller LH, Becker DJ, Pittsburgh Epidemiology of Diabetes Complications Study. Lipid and blood pressure treatment goals for type 1 diabetes: 10-year incidence data from the Pittsburgh Epidemiology of Diabetes Complications Study. Diabetes Care 2001;24:1053-1059

321. Kavey RE, Allada V, Daniels SR, Hayman LL, McCrindle BW, Newburger JW, Parekh RS, Steinberger J, American Heart Association Expert Panel on Population and Prevention Science, American Heart Association Council on Cardiovascular Disease in the Young, American Heart Association Council on Epidemiology and Prevention, American Heart Association Council on Nutrition, Physical Activity and Metabolism, American Heart Association Council on High Blood Pressure Research, American Heart Association Council on Cardiovascular Nursing, American Heart Association Council on the Kidney in Heart Disease, Interdisciplinary Working Group on Quality of Care and Outcomes Research. Cardiovascular risk reduction in high-risk pediatric patients: a scientific statement from the American Heart Association Expert Panel on Population and Prevention Science; the Councils on Cardiovascular Disease in the Young, Epidemiology and Prevention, Nutrition, Physical Activity and Metabolism, High Blood Pressure Research, Cardiovascular Nursing, and the Kidney in Heart Disease; and the Interdisciplinary Working Group on Quality of Care and Outcomes Research: endorsed by the American Academy of Pediatrics. Circulation 2006;114:27102738

322. McCrindle BW, Urbina EM, Dennison BA, Jacobson MS, Steinberger J, Rocchini AP, Hayman LL, Daniels SR, American Heart Association Atherosclerosis,
Hypertension, and Obesity in Youth Committee, American Heart Association Council of Cardiovascular Disease in the Young, American Heart Association Council on Cardiovascular Nursing. Drug therapy of high-risk lipid abnormalities in children and adolescents: a scientific statement from the American Heart Association Atherosclerosis, Hypertension, and Obesity in Youth Committee, Council of Cardiovascular Disease in the Young, with the Council on Cardiovascular Nursing. Circulation 2007;115:1948-1967

323. Salo P, Viikari J, Hämäläinen M, Lapinleimu H, Routi T, Rönnemaa T, Seppänen R, Jokinen E, Välimäki I, Simell $\mathrm{O}$. Serum cholesterol ester fatty acids in 7- and 13-month-old children in a prospective randomized trial of a low-saturated fat, low-cholesterol diet: the STRIP baby project. Special Turku coronary Risk factor Intervention Project for children. Acta Paediatr 1999;88:505-512

324. Efficacy and safety of lowering dietary intake of fat and cholesterol in children with elevated low-density lipoprotein cholesterol. The Dietary Intervention Study in Children (DISC). The Writing Group for the DISC Collaborative Research Group. JAMA 1995;273:14291435

325. McCrindle BW, Ose L, Marais AD. Efficacy and safety of atorvastatin in children and adolescents with familial hypercholesterolemia or severe hyperlipidemia: a multicenter, randomized, placebo-controlled trial. J Pediatr 2003; 143:74-80

326. de Jongh S, Lilien MR, op't RJ, Stroes ES, Bakker HD, Kastelein JJ. Early statin therapy restores endothelial function in children with familial hypercholesterolemia. J Am Coll Cardiol 2002;40:21172121

327. Wiegman A, Hutten BA, de Groot E, Rodenburg J, Bakker HD, Büller HR, Sijbrands EJ, Kastelein JJ. Efficacy and safety of statin therapy in children with familial hypercholesterolemia: a randomized controlled trial. JAMA 2004; 292:331-337

328. Holmes GK. Screening for coeliac disease in type 1 diabetes. Arch Dis Child 2002;87:495-498

329. Rewers M, Liu E, Simmons J, Redondo MJ, Hoffenberg EJ: Celiac disease associated with type 1 diabetes mellitus. Endocrinol Metab Clin North Am 2004;33: 197-214, xi

330. Roldan MB, Alonso M, Barrio R. Thyroid autoimmunity in children and adolescents with Type 1 diabetes mellitus. Diabetes Nutr Metab 1999;12:27-31

331. Kordonouri O, Deiss D, Danne $T$, Dorow A, Bassir C, Grüters-Kieslich A. Predictivity of thyroid autoantibodies for the development of thyroid disorders in children and adolescents with Type 1 diabetes. Diabet Med 2002;19:518-521

332. Mohn A, Di Michele S, Di Luzio R, Tumini S, Chiarelli F. The effect of subclinical hypothyroidism on metabolic control in children and adolescents with Type 1 diabetes mellitus. Diabet Med 2002; 19:70-73

333. Chase HP, Garg SK, Cockerham RS, Wilcox WD, Walravens PA. Thyroid hormone replacement and growth of children with subclinical hypothyroidism and diabetes. Diabet Med 1990;7: 299-303

334. Eppens MC, Craig ME, Cusumano J, Hing S, Chan AK, Howard NJ, Silink M, Donaghue KC. Prevalence of diabetes complications in adolescents with type 2 compared with type 1 diabetes. Diabetes Care 2006;29:1300-1306

335. Kitzmiller JL, Gavin LA, Gin GD, Jovanovic-Peterson L, Main EK, Zigrang WD. Preconception care of diabetes. Glycemic control prevents congenital anomalies. JAMA 1991;265:731-736

336. Goldman JA, Dicker D, Feldberg D, Yeshaya A, Samuel N, Karp M. Pregnancy outcome in patients with insulin-dependent diabetes mellitus with preconceptional diabetic control: a comparative study. AmJ Obstet Gynecol 1986;155: 293-297

337. Rosenn B, Miodovnik M, Combs CA, Khoury J, Siddiqi TA. Pre-conception management of insulin-dependent diabetes: improvement of pregnancy outcome. Obstet Gynecol 1991;77:846849

338. Tchobroutsky C, Vray MM, Altman JJ. Risk/benefit ratio of changing late obstetrical strategies in the management of insulin-dependent diabetic pregnancies. A comparison between 1971-1977 and 1978-1985 periods in 389 pregnancies. Diabete Metab 1991;17:287-294

339. Willhoite MB, Bennert HW, Jr, Palomaki GE, Zaremba MM, Herman WH, Williams JR, Spear NH. The impact of preconception counseling on pregnancy outcomes. The experience of the Maine Diabetes in Pregnancy Program. Diabetes Care 1993; 16:450-455

340. Cooper WO, Hernandez-Diaz S, Arbogast PG, Dudley JA, Dyer S, Gideon PS, Hall K, Ray WA. Major congenital malformations after first-trimester exposure to ACE inhibitors. N Engl J Med 2006; 354:2443-2451

341. American Diabetes Association: Preconception care of women with diabetes (Position Statement). Diabetes Care 2004;27(Suppl. 1):S76-S78

342. Brown AF, Mangione CM, Saliba D, Sarkisian CA, California Healthcare Foundation/American Geriatrics Society Panel on Improving Care for Elders with Diabetes. Guidelines for improving the care of the older person with diabetes 
mellitus. J Am Geriatr Soc 2003;51: S265-S280

343. Curb JD, Pressel SL, Cutler JA, Savage PJ, Applegate WB, Black H, Camel G, Davis BR, Frost PH, Gonzalez N, Guthrie G, Oberman A, Rutan GH, Stamler J. Effect of diuretic-based antihypertensive treatment on cardiovascular disease risk in older diabetic patients with isolated systolic hypertension. Systolic Hypertension in the Elderly Program Cooperative Research Group. JAMA 1996;276: 1886-1892

344. Beckett NS, Peters R, Fletcher AE, Staessen JA, Liu L, Dumitrascu D, Stoyanovsky V, Antikainen RL, Nikitin Y, Anderson C, Belhani A, Forette F, Rajkumar C, Thijs L, Banya W, Bulpitt CJ, HYVET Study Group. Treatment of hypertension in patients 80 years of age or older. N Engl J Med 2008;358:1887-1898

345. Clement S, Braithwaite SS, Magee MF, Ahmann A, Smith EP, Schafer RG, Hirsch IB, Hirsh IB, American Diabetes Association Diabetes in Hospitals Writing Committee. Management of diabetes and hyperglycemia in hospitals. Diabetes Care 2004:27:553-591

346. Moghissi ES, Korytkowski MT, DiNardo M, Einhorn D, Hellman R, Hirsch IB, Inzucchi SE, Ismail-Beigi F, Kirkman MS, Umpierrez GE, American Association of Clinical Endocrinologists, American Diabetes Association. American Association of Clinical Endocrinologists and American Diabetes Association consensus statement on inpatient glycemic control. Diabetes Care 2009;32: 1119-1131

347. American Diabetes Association: Economic costs of diabetes in the U.S. In 2007. Diabetes Care 2008;31:596-615

348. Centers for Disease Control and Prevention. National Diabetes Fact Sheet: General Information and National Estimates on Diabetes in the United States, 2007. Atlanta, GA, US Department of Health and Human Services, 2008

349. Levetan CS, Passaro M, Jablonski K, Kass M, Ratner RE. Unrecognized diabetes among hospitalized patients. Diabetes Care 1998;21:246-249

350. Umpierrez GE, Isaacs SD, Bazargan N, You X, Thaler LM, Kitabchi AE. Hyperglycemia: an independent marker of inhospital mortality in patients with undiagnosed diabetes. J Clin Endocrinol Metab 2002;87:978-982

351. van den Berghe G, Wouters P, Weekers F, Verwaest C, Bruyninckx F, Schetz M, Vlasselaers D, Ferdinande P, Lauwers P, Bouillon R. Intensive insulin therapy in the critically ill patients. N Engl J Med 2001;345:1359-1367

352. Malmberg K, Norhammar A, Wedel H, Rydén L. Glycometabolic state at admission: important risk marker of mortality in conventionally treated patients with dia- betes mellitus and acute myocardial infarction: long-term results from the Diabetes and Insulin-Glucose Infusion in Acute Myocardial Infarction (DIGAMI) study. Circulation 1999;99:2626-2632

353. Wiener RS, Wiener DC, Larson RJ. Benefits and risks of tight glucose control in critically ill adults: a meta-analysis. JAMA 2008;300:933-944

354. Brunkhorst FM, Engel C, Bloos F, MeierHellmann A, Ragaller M, Weiler N, Moerer O, Gruendling M, Oppert M, Grond S, Olthoff D, Jaschinski U, John S, Rossaint R, Welte T, Schaefer M, Kern P, Kuhnt E, Kiehntopf M, Hartog C, Natanson C, Loeffler M, Reinhart K, German Competence Network Sepsis (SepNet). Intensive insulin therapy and pentastarch resuscitation in severe sepsis. N Engl J Med 2008;358:125-139

355. NICE-SUGAR Study Investigators, Finfer S, Chittock DR, Su SY, Blair D, Foster D, Dhingra V, Bellomo R, Cook D, Dodek P, Henderson WR, Hébert PC, Heritier S, Heyland DK, McArthur C, McDonald E, Mitchell I, Myburgh JA, Norton R, Potter J, Robinson BG, Ronco JJ. Intensive versus conventional glucose control in critically ill patients. N Engl J Med 2009;360:1283-1297

356. Krinsley JS, Grover A. Severe hypoglycemia in critically ill patients: risk factors and outcomes. Crit Care Med 2007;35: 2262-2267

357. Van den Berghe G, Wilmer A, Hermans G, Meersseman W, Wouters PJ, Milants I, Van Wijngaerden E, Bobbaers $\mathrm{H}$, Bouillon R. Intensive insulin therapy in the medical ICU. N Engl J Med 2006; 354:449-461

358. Griesdale DE, de Souza RJ, van Dam RM, Heyland DK, Cook DJ, Malhotra A, Dhaliwal R, Henderson WR, Chittock DR, Finfer S, Talmor D. Intensive insulin therapy and mortality among critically ill patients: a meta-analysis including NICE-SUGAR study data. CMAJ 2009; 180:821-827

359. Saudek CD, Herman WH, Sacks DB, Bergenstal RM, Edelman D, Davidson MB. A new look at screening and diagnosing diabetes mellitus. J Clin Endocrinol Metab 2008;93:2447-2453

360. Mitrakou A, Ryan C, Veneman T, Mokan M, Jenssen T, Kiss I, Durrant J, Cryer P, Gerich J. Hierarchy of glycemic thresholds for counterregulatory hormone secretion, symptoms, and cerebral dysfunction. Am J Physiol 1991;260:E67-E74

361. Korytkowski M, DiNardo M, Donihi AC, Bigi L, Devita M. Evolution of a diabetes inpatient safety committee. Endocr Pract 2006;12(Suppl. 3):91-99

362. DiNardo M, Noschese M, Korytkowski $\mathrm{M}$, Freeman S. The medical emergency team and rapid response system: finding, treating, and preventing hypoglycemia. Jt Comm J Qual Patient Saf 2006;
32:591-595

363. Donihi AC, Raval D, Saul M, Korytkowski MT, DeVita MA. Prevalence and predictors of corticosteroid-related hyperglycemia in hospitalized patients. Endocr Pract 2006;12:358-362

364. Goldberg PA, Siegel MD, Sherwin RS, Halickman JI, Lee M, Bailey VA, Lee SL, Dziura JD, Inzucchi SE. Implementation of a safe and effective insulin infusion protocol in a medical intensive care unit. Diabetes Care 2004;27:461-467

365. Rea RS, Donihi AC, Bobeck M, Herout P, McKaveney TP, Kane-Gill SL, Korytkowski MT. Implementing an intravenous insulin infusion protocol in the intensive care unit. Am J Health Syst Pharm 2007;64:385-395

366. Nazer LH, Chow SL, Moghissi ES. Insulin infusion protocols for critically ill patients: a highlight of differences and similarities. Endocr Pract 2007;13:137146

367. DeSantis AJ, Schmeltz LR, Schmidt K, O'Shea-Mahler E, Rhee C, Wells A, Brandt S, Peterson S, Molitch ME. Inpatient management of hyperglycemia: the Northwestern experience. Endocr Pract 2006;12:491-505

368. Noschese M, Donihi AC, Koerbel G, Karslioglu E, DiNardo M, Curll M, Korytkowski MT. Effect of a diabetes order set on glycaemic management and control in the hospital. Qual Saf Health Care 2008; 17:464-468

369. Umpierrez GE, Hor T, Smiley D, Temponi A, Umpierrez D, Ceron M, Munoz C, Newton C, Peng L, Baldwin D. Comparison of inpatient insulin regimens with detemir plus aspart versus neutral protamine hagedorn plus regular in medical patients with type 2 diabetes. J Clin Endocrinol Metab 2009;94:564569

370. Moghissi ES, Hirsch IB. Hospital management of diabetes. Endocrinol Metab Clin North Am 2005;34:99-116

371. Umpierrez GE, Smiley D, Zisman A, Prieto LM, Palacio A, Ceron M, Puig A, Mejia R. Randomized study of basal-bolus insulin therapy in the inpatient management of patients with type 2 diabetes (RABBIT 2 trial). Diabetes Care 2007;30: 2181-2186

372. Umpierrez GE, Palacio A, Smiley D. Sliding scale insulin use: myth or insanity? Am J Med 2007;120:563-567

373. Queale WS, Seidler AJ, Brancati FL. Glycemic control and sliding scale insulin use in medical inpatients with diabetes mellitus. Arch Intern Med 1997;157: 545-552

374. Korytkowski MT, Salata RJ, Koerbel GL, Selzer F, Karslioglu E, Idriss AM, Lee KK, Moser AJ, Toledo FG. Insulin therapy and glycemic control in hospitalized patients with diabetes during enteral nutrition therapy: a randomized controlled 
clinical trial. Diabetes Care 2009;32: 594-596

375. Hirsch IB. Sliding scale insulin-time to stop sliding. JAMA 2009;301:213-214

376. Umpierrez GE. Basal versus sliding-scale regular insulin in hospitalized patients with hyperglycemia during enteral nutrition therapy. Diabetes Care 2009;32: 751-753

377. Pancorbo-Hidalgo PL, García-Fernandez FP, Ramírez-Pérez C. Complications associated with enteral nutrition by nasogastric tube in an internal medicine unit. J Clin Nurs 2001;10:482-490

378. Nylen ES, Muller B. Endocrine changes in critical illness. J Intensive Care Med 2004;19:67-82

379. Shilo S, Berezovsky S, Friedlander Y, Sonnenblick M. Hypoglycemia in hospitalized nondiabetic older patients. J Am Geriatr Soc 1998;46:978-982

380. Fischer KF, Lees JA, Newman JH. Hypoglycemia in hospitalized patients. Causes and outcomes. N Engl J Med 1986;315:1245-1250

381. Markovitz LJ, Wiechmann RJ, Harris N, Hayden V, Cooper J, Johnson G, Harelstad R, Calkins L, Braithwaite SS. Description and evaluation of a glycemic management protocol for patients with diabetes undergoing heart surgery. Endocr Pract 2002;8:10-18

382. Levetan CS, Salas JR, Wilets IF, Zumoff B. Impact of endocrine and diabetes team consultation on hospital length of stay for patients with diabetes. Am J Med 1995;99:22-28

383. Levetan CS, Passaro MD, Jablonski KA, Ratner RE. Effect of physician specialty on outcomes in diabetic ketoacidosis. Diabetes Care 1999;22:1790-1795

384. Koproski J, Pretto Z, Poretsky L. Effects of an intervention by a diabetes team in hospitalized patients with diabetes. Diabetes Care 1997;20:1553-1555

385. Furnary AP, Braithwaite SS. Effects of outcome on in-hospital transition from intravenous insulin infusion to subcutaneous therapy. Am J Cardiol 2006;98: 557-564

386. American Diabetes Association: Diabetes nutrition recommendations for health care institutions (Position Statement). Diabetes Care 2004;27(Suppl. 1):S55-S57

387. Boucher JL, Swift CS, Franz MJ, Kulkarni K, Schafer RG, Pritchett E, Clark NG. Inpatient management of diabetes and hyperglycemia: implications for nutrition practice and the food and nutrition professional. J Am Diet Assoc 2007;107:105-111

388. Scott MG, Bruns DE, Boyd JC, Sacks DB. Tight glucose control in the intensive care unit: are glucose meters up to the task? Clin Chem 2009;55:18-20
389. D'Orazio P, Burnett RW, FoghAndersen N, Jacobs E, Kuwa K, Külpmann WR, Larsson L, Lewenstam A, Maas AH, Mager G, Naskalski JW, Okorodudu AO, International Federation of Clinical Chemistry Scientific Division Working Group on Selective Electrodes and Point of Care Testing. Approved IFCC recommendation on reporting results for blood glucose (abbreviated). Clin Chem 2005;51:1573-1576

390. Dungan K, Chapman J, Braithwaite SS, Buse J. Glucose measurement: confounding issues in setting targets for inpatient management. Diabetes Care 2007;30:403-409

391. Boyd JC, Bruns DE. Quality specifications for glucose meters: assessment by simulation modeling of errors in insulin dose. Clin Chem 2001;47:209-214

392. Goldberg PA, Siegel MD, Russell RR, Sherwin RS, Halickman JI, Cooper DA, Dziura JD, Inzucchi SE. Experience with the continuous glucose monitoring system in a medical intensive care unit. Diabetes Technol Ther 2004;6:339-347

393. Cheung BM, Ong KL, Cherny SS, Sham PC, Tso AW, Lam KS. Diabetes prevalence and therapeutic target achievement in the United States, 1999 to 2006. Am J Med 2009; 122:443-453

394. Coleman K, Austin BT, Brach C, Wagner EH. Evidence on the Chronic Care Model in the new millennium. Health Aff (Millwood) 2009;28:75-85

395. Clark CM, Jr, Snyder JW, Meek RL, Stutz LM, Parkin CG. A systematic approach to risk stratification and intervention within a managed care environment improves diabetes outcomes and patient satisfaction. Diabetes Care 2001;24:1079_ 1086

396. Meigs JB, Cagliero E, Dubey A, MurphySheehy P, Gildesgame C, Chueh H, Barry MJ, Singer DE, Nathan DM. A controlled trial of web-based diabetes disease management: the MGH diabetes primary care improvement project. Diabetes Care 2003:26:750-757

397. O'Connor PJ, Desai J, Solberg LI, Reger LA, Crain AL, Asche SE, Pearson TL, Clark CK, Rush WA, Cherney LM, Sperl-Hillen JM, Bishop DB. Randomized trial of quality improvement intervention to improve diabetes care in primary care settings. Diabetes Care 2005;28:1890-1897

398. Sperl-Hillen JM, O'Connor PJ. Factors driving diabetes care improvement in a large medical group: ten years of progress. Am J Manag Care 2005;11: S177-S185

399. Siminerio LM. Implementing diabetes self-management training programs: breaking through the barriers in primary care. EndocrPract 2006;12(Suppl.
1):124-130

400. Mahoney JJ. Reducing patient drug acquisition costs can lower diabetes health claims. Am J Manag Care 2005;11: S170-S176

401. Maney M, Tseng CL, Safford MM, Miller DR, Pogach LM. Impact of self-reported patient characteristics upon assessment of glycemic control in the Veterans Health Administration. Diabetes Care 2007;30:245-251

402. Bergenstal RM. Treatment models from the International Diabetes Center: advancing from oral agents to insulin therapy in type 2 diabetes. Endocr Pract 2006;12(Suppl. 1):98-104

403. Berikai P, Meyer PM, Kazlauskaite R, Savoy B, Kozik K, Fogelfeld L. Gain in patients' knowledge of diabetes management targets is associated with better glycemic control. Diabetes Care 2007; 30:1587-1589

404. O'Connor PJ. Electronic medical records and diabetes care improvement: are we waiting for Godot? Diabetes Care 2003; 26:942-943

405. Shojania KG, Ranji SR, McDonald KM, Grimshaw JM, Sundaram V, Rushakoff RJ, Owens DK. Effects of quality improvement strategies for type 2 diabetes on glycemic control: a meta-regression analysis. JAMA 2006;296:427-440

406. Davidson MB. How our current medical care system fails people with diabetes: lack of timely, appropriate clinical decisions. Diabetes Care 2009;32:370-372

407. McLean DL, McAlister FA, Johnson JA, King KM, Makowsky MJ, Jones CA, Tsuyuki RT, SCRIP-HTN Investigators. A randomized trial of the effect of community pharmacist and nurse care on improving blood pressure management in patients with diabetes mellitus: study of cardiovascular risk intervention by pharmacists-hypertension (SCRIP-HTN). Arch Intern Med 2008;168:2355-2361

408. Parchman ML, Zeber JE, Romero RR, Pugh JA. Risk of coronary artery disease in type 2 diabetes and the delivery of care consistent with the chronic care model in primary care settings: a STARNet study. Med Care 2007:45:1129_ 1134

409. Feifer C, Nemeth L, Nietert PJ, Wessell AM, Jenkins RG, Roylance L, Ornstein SM. Different paths to high-quality care: three archetypes of top-performing practice sites. Ann Fam Med 2007;5: 233-241

410. Ornstein S, Nietert PJ, Jenkins RG, Wessell AM, Nemeth LS, Feifer C, Corley ST. Improving diabetes care through a multicomponent quality improvement model in a practice-based research network. Am J Med Qual 2007;22:34-41 